\title{
Development of Polypyrrole Encapsulated Nanomaterials in a Syringe Needle for Automated Molecularly Imprinted Solid Phase Extraction of Ochratoxin A
}

\author{
By \\ Yun Wei, M.Sc. \\ A Thesis Submitted to the Faculty of Graduate Studies and Research in \\ Partial Fulfillment of the Requirements for the Degree of Master of Science
}

\author{
Department of Chemistry \\ Carleton University \\ Ottawa, Ontario
}

June 2007

(a) Copyright

Yun Wei 


$\begin{array}{ll}\begin{array}{l}\text { Library and } \\ \text { Archives Canada }\end{array} & \begin{array}{l}\text { Bibliothèque et } \\ \text { Archives Canada }\end{array} \\ \begin{array}{l}\text { Published Heritage } \\ \text { Branch }\end{array} & \begin{array}{l}\text { Direction du } \\ \text { Patrimoine de l'édition }\end{array} \\ \begin{array}{l}\text { 395 Wellington Street } \\ \text { Ottawa ON K1A ON4 }\end{array} & \begin{array}{l}\text { 395, rue Wellington } \\ \text { Ottawa ON K1A ON4 } \\ \text { Canada }\end{array}\end{array}$

Your file Votre référence ISBN: 978-0-494-33716-5 Our file Notre référence ISBN: 978-0-494-33716-5

NOTICE:

The author has granted a nonexclusive license allowing Library and Archives Canada to reproduce, publish, archive, preserve, conserve, communicate to the public by telecommunication or on the Internet, loan, distribute and sell theses worldwide, for commercial or noncommercial purposes, in microform, paper, electronic and/or any other formats.

The author retains copyright ownership and moral rights in this thesis. Neither the thesis nor substantial extracts from it may be printed or otherwise reproduced without the author's permission.
AVIS:

L'auteur a accordé une licence non exclusive permettant à la Bibliothèque et Archives Canada de reproduire, publier, archiver, sauvegarder, conserver, transmettre au public par télécommunication ou par l'Internet, prêter, distribuer et vendre des thèses partout dans le monde, à des fins commerciales ou autres, sur support microforme, papier, électronique et/ou autres formats.

L'auteur conserve la propriété du droit d'auteur et des droits moraux qui protège cette thèse. $\mathrm{Ni}$ la thèse ni des extraits substantiels de celle-ci ne doivent être imprimés ou autrement reproduits sans son autorisation.
In compliance with the Canadian

Privacy Act some supporting forms may have been removed from this thesis.

While these forms may be included in the document page count, their removal does not represent any loss of content from the thesis.
Conformément à la loi canadienne sur la protection de la vie privée, quelques formulaires secondaires ont été enlevés de cette thèse.

Bien que ces formulaires aient inclus dans la pagination, il n'y aura aucun contenu manquant.

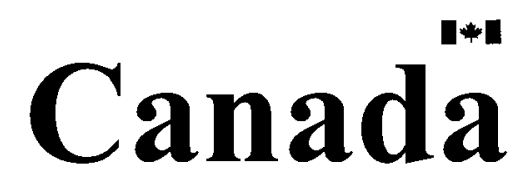




\begin{abstract}
Two kinds of nano-materials, carbon nanotubes and quantum dots, were explored to form molecularly imprinted polypyrrole encapsulated nano-composites for use as micro molecularly imprinted solid phase extraction sorbents. The sorbent was prepared inside a stainless steel syringe needle via in-situ electropolymerization of pyrrole. By coupling the modified needle to an autosampler of high performance liquid chromatography, a new configuration of automated method for rapid determination of Ochratoxin $\mathrm{A}$ in red wine was established. The configuration facilitated the integration of sampling (extraction) /pulsed elution (PE)/injection (detection) into one closed system.

A good linearity $\left(\mathrm{R}^{2}=0.9937\right)$ was achieved over the Ochratoxin A concentration range of 0.02 to $3.0 \mathrm{ng} / \mathrm{mL}$, with a limit of detection of $0.02 \mathrm{ppb}$ or a limit of quantification of $0.06 \mathrm{ppb}$. The total automated analysis took 20 minutes and only $1.0 \mathrm{~mL}$ sample was required. The result of differential pulsed elution demonstrated that the sorbent has specific recognition for Ochratoxin A.
\end{abstract}




\section{ACKNOWLEDGEMENTS}

I would like to express my thanks to all those who helped and supported me to complete this thesis.

First of all, my sincerest appreciation goes to Dr. Edward P. C. Lai, my supervisor, who gave me incredible support, expertise, encouragement and patience throughout my research work. My gratitude is not only for his profound knowledge and critical academic advice but also for his hard-working spirit and strict attitude towards scientific research. He could not even realize how much I have learned from him during my M. Sc. studies at Carleton University and how much it will benefit me in my future career.

I am very grateful to Dr. R. Burk and Dr. P. Sundararajan who agreed to be members of my thesis advisory committee as well as, Jim Logan, who helped me immensely in resolving my electronic and mechanical problems during my research work, and Fred Cassalman, who made my TA job more enjoyable.

I would also like to extend my thanks to our group members, Mark Pereira, Jorn $\mathrm{Yu}$, Erika Sanger, Erika Revesz, and Amy Hrdina who shared their experience, skills and wisdom with me.

Finally, thanks my family for their support, patience and endless love. My husband Longhui Qiu gave me continuous encouragement throughout my thesis. I also own thanks to my dear mother, who devoted her time to looking after my family, so I could pursue my studies. I also thank my daughter Yuelin, who did her best to help take care of her brother and who tried very hard to do her school work on her own. Special thanks to my deceased farther who taught me the values of self-responsibility and positive attitude towards work and life. 


\section{Table of Contents}

Title page

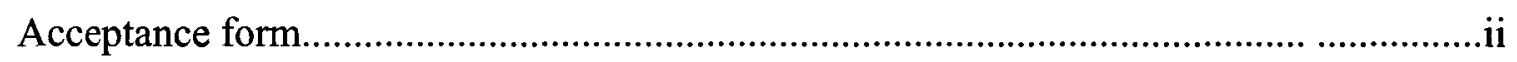

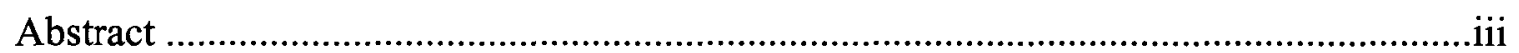

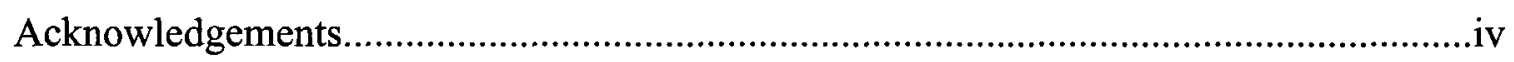

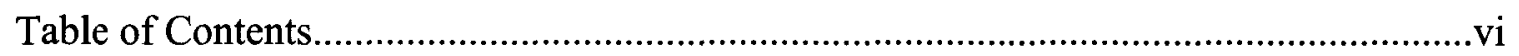

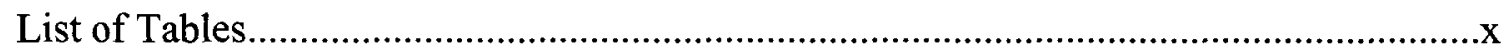

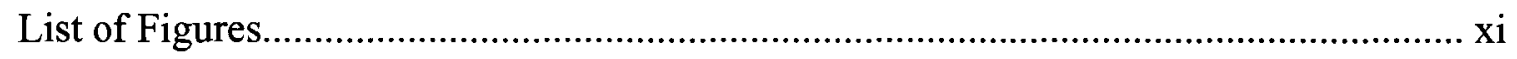

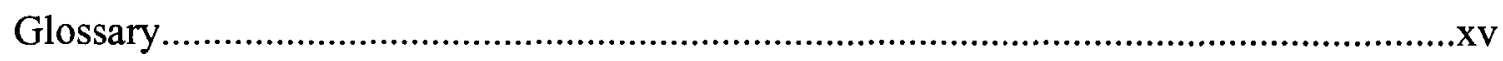

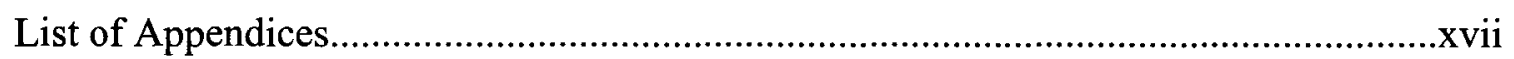




\section{Chapter I Introduction}

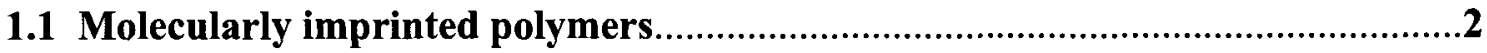

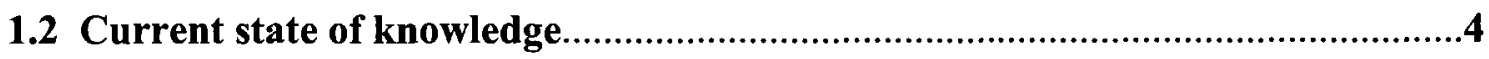

1.2.1 Development of molecular imprinting technology.................................

1.2.2 Theory of molecularly imprinted polymers............................................5

1.2.3 Conventional preparation of molecularly imprinted polymers..................7

1.2.4 Molecularly imprinted polymers for solid phase extraction......................8

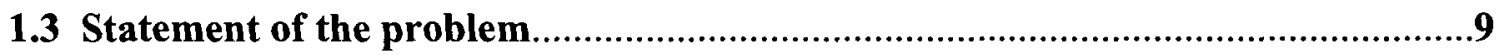

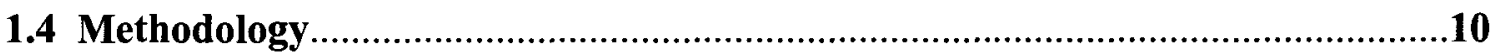

1.4.1 Molecularly imprinted polypyrrole based nano-composites......................10

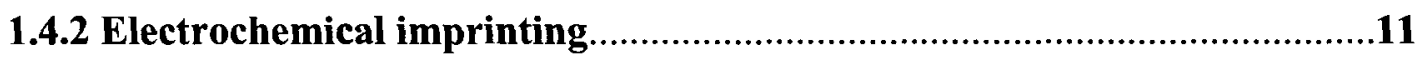

1.4.3 Pulsed elution and differential pulsed elution......................................12

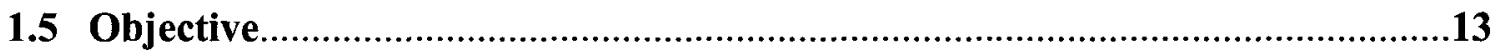

\section{Chapter II Experimental Section}

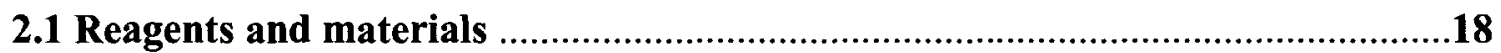

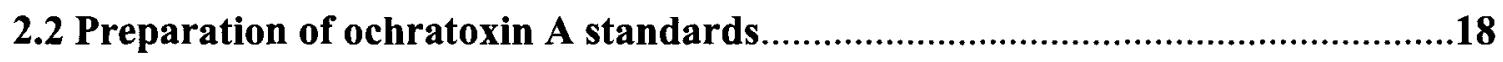

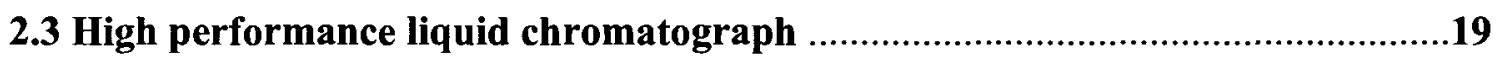

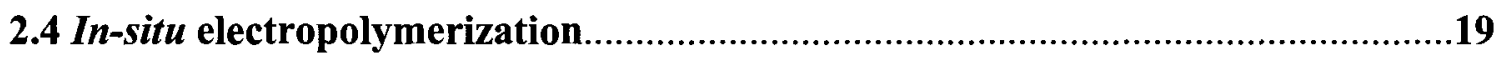

2.5 Removal of the template OTA from the modified needle ...................................20

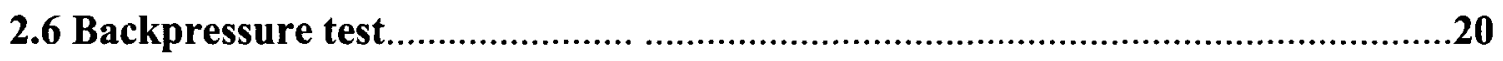

2.7 Off-line MIPPy/CNTs- $\mu$ SPE-PE-HPLC-FD analysis ....................................21

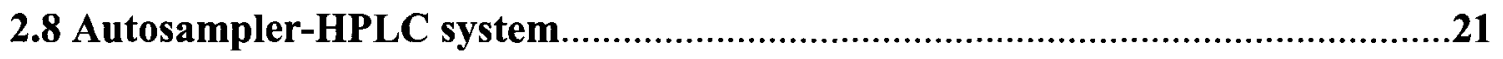

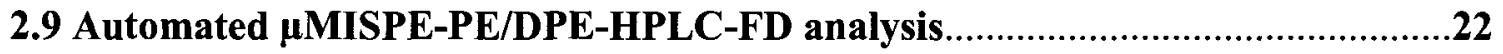


2.9.1 Sampling (extraction)

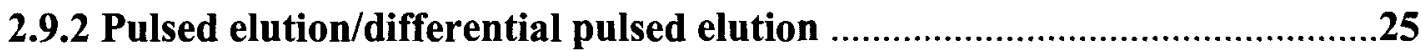

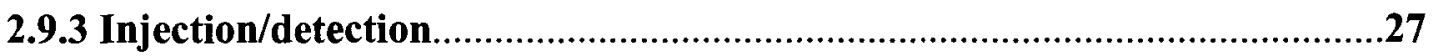

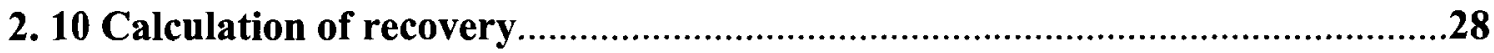

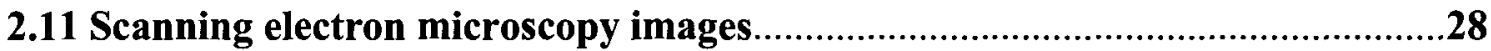

CHAPTER III Molecularly Imprinted Solid Phase Extraction in a Syringe

Needle packed with Polypyrrole-encapsulated Carbon Nanotubes

for Determination of Ochratoxin A in Red Wine

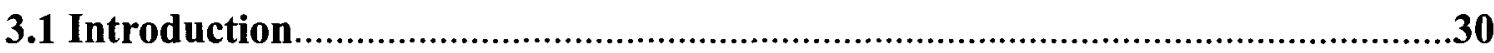

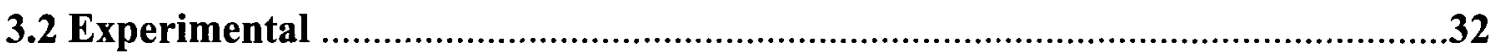

3.2.1 Optimization of the electropolymerization time....................................32

3.2.2 Choose of the eluting solvent for removal of temple OTA.........................32

3.2.3 Effect of sample pH on MIPPy/CNTs- $\mu$ SPE-PE-HPLC-FD ....................32

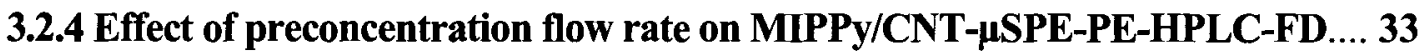

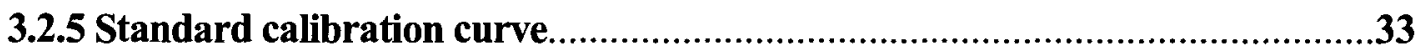

3.2.6 Application of MIPPy/CNTs- $\mu$ SPE-PE-HPLC-FD to red wine analysis...........34

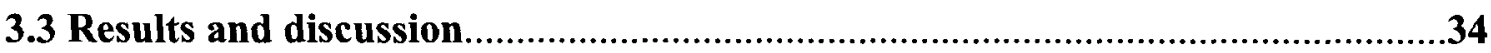

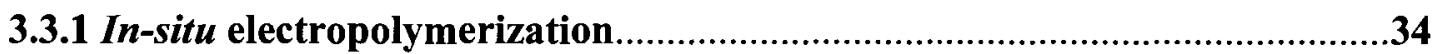

3.3.2 Optimization of the electropolymerization time.......................................35

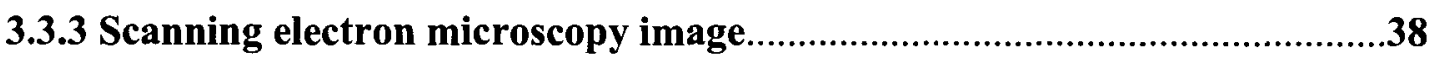

3.3.4 Choose of the eluting solvent for removal of temple OTA.........................38

3.3.5 Effect of sample pH on MIPPy/CNT- $\mu$ SPE-PE-HPLC-FD ......................41

3.3.6 Effect of preconcentration flow rate on MIPPy/CNT- $\mu$ SPE-HPLC-FD.....43

vii 
3.3.7 Standard calibration curve. .45

3.3.8 Application of MIPPy/CNTs- $\mu$ SPE-PE-HPLC-FD to red wine analysis... 46

3.3.9 Reusability of MIPPy/CNTs-modified syringe needles 47

3.4 Conclusion. 48

Chapter IV Encapsulation of Quantum Dots and Carbon Nanotubes with Polypyrrole in a Syringe Needle for Automated Molecularly Imprinted Solid Phase Extraction of Ochratoxin A in Red Wine Analysis

4.1 Introduction .50

4.2 Experimental .53

4.2.1 Preparation of MIPPy/CNTs/QDs-modified stainless steel syringe needle.

4.2.2 Measurement of total surface area by pulsed amperometry 54

4.2.3 Effect of analyte OTA concentration on recovery of MIPPy/CNTs/QDsMSPE-PE-HPLC-FD .56

4.2.4 Standard calibration curve for MIPPy/CNTs/QDs- $\mu$ SPE-PE-HPLC-FD analysis of OTA

4.2.5 Automated direct HPLC-FD analysis of red wine for OTA

4.2.6 Automated MIPPy/CNTs/QDs- $\mu$ SPE-PE-HPLC-FD analysis of red wine for OTA

4.2.7 Choose of eluting solvent for differential pulsed elution. 58

4.2.8 Automated MIPPy/CNTs/QDs- $\mu$ SPE-DPE-HPLC-FD analysis of red wine for OTA .58

4.3 Results and Discussion .58

viii 
4.3.1 Autosampler system for MIPPy/CNTs/QDs- $\mu$ SPE-PE-HPLC-FD

analysis .58

4.3.2 Preparation of MIPPy/CNTs/QDs-modified stainless steel needles. 60

4.3.3 Scanning electron microscopy image .61

4.3.4 Optimization of template OTA concentration in pre-polymerization mixture .63

4.3.5 Effect of analyte OTA concentration on recovery of automated MIPPy/CNTs/QDs- $\mu$ SPE-PE-HPLC-FD .65

4.3.6 Standard calibration curve for automated MIPPy/CNTs/QDs- $\mu$ SPE-PEHPLC-FD analysis. .66

4.3.7 Measurement of total surface area by pulsed amperometry. .67

4.3.8 Effect of surface area over the recovery 69

4.3.9 Automated MIPPy/CNTs/QDs- $\mu$ SPE-PE-HPLC-FD analysis of red wine. .69

4.3.10 Improvement in recovery of OTA in red wine analysis. .72

4.3.11 Elimination of non-specific binding of red wine matrix components with differential pulsed elution. .73

4.3.12 Effect of different red wine matrix .76

4.4 Conclusions. .80

\section{Chapter IV Conclusion}

Conclusion .82

\section{Reference}

Reference 86 


\section{LIST OF TABLES}

Table 3-1 Electropolymerization time and backpressure test...................................37

Table 3-2 Choose of eluting solvent for removal of template......................................41

Table 4-1 Parameters used in the PalmTime program for PAD analysis (version 1.35)

TableA-1 System configuration at the sample uptake step $(1.0 \mathrm{~mL}$ sample $) .. \ldots \ldots \ldots \ldots \ldots . . . .107$

TableA-2 System configuration for pulsed elution $(0.2 \mathrm{~mL}$ eluting solution each time) .110

TableA-3 Manual injection table for pulsed elution ( $0.2 \mathrm{~mL}$ eluting solvent each time) 


\section{LIST OF FIGURES}

Fig. 1-1 Schematic principle of molecular imprinting.............................................

Fig. 1-2 Supposed model of Py cross-linked by EDGMA [40] ................................11

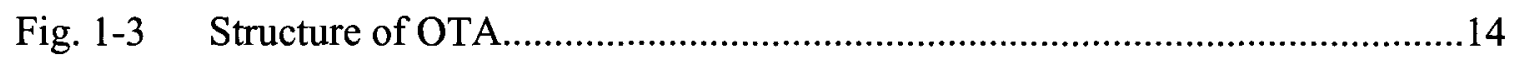

Fig. 2-1 Preparation of MIPPy/CNTs-modified needle by electropolymerization........20

Fig. 2-2 Schematic diagram of the connection of $\mu$ MISPE sorbent packed needle with

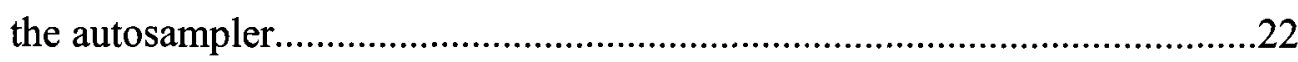

Fig. 2-3 Schematic diagram of in-needle $\mu$ MISPE...............................................23

Fig. 2-4(a) Schematic diagram of automated $\mu$ MISPE-PE/DPE-HPLC-FD analysis in step 1-sampling (extraction). The modified needle is uptaking $1.0 \mathrm{~mL}$ of sample from vial

Fig. 2-4(b) Schematic diagram of automated $\mu$ MISPE-PE/DPE-HPLC-FD analysis in step 1-sampling (extraction). Sample loop and column are being washed with HPLC mobile phase. .25

Fig. 2-5 Schematic diagram of automated $\mu$ MISPE-PE/DPE-HPLC-FD analysis in step 2-pulsed elution. The extracted OTA is being washed off the syringe needle with $2 \%$ TEA (prepared in HPLC mobile phase) and the eluate is collected with the automated injection valve of the autosampler system and loaded onto the sample loop. .26

Fig. 2-6 Schematic diagram of automated $\mu$ MISPE-PE/DPE-HPLC-FD analysis in step 3 -injection/detection. The eluate is injected onto the column from the sample loop. The sample loop and column are being washed with HPLC mobile phase. 
Fig. 3-1 SEM of MIPPy/CNTs on the inner wall of a modified stainless steel syringe needle. (a) Tip of the modified needle; (b) Red circled area in (a) under higher magnification

Fig. 3-2 Residue template in the polymeric structure of bulk MIPs

G: residue template (modified from reference 24) 39

Fig. 3-3 Effect of sample $\mathrm{pH}$ on MIPPy/CNT- $\mu$ SPE-PE-HPLC-FD

Fig. 3-4 Effect of pre-concentration flow rate on MIPPy/CNT- $\mu$ SPE-PE-HPLC-FD

Fig. 3-5 Standard calibration curve of MIPPy/CNTs- $\mu$ SPE-PE-HPLC-FD analysis with spiked red wine samples.

Fig. 3-6 Analysis of 0.5 ppb-OTA-spiked red wines by MIPPy/CNTs- $\mu$ SPE-PE-HPLCFD after enrichment of OTA from $0.5 \mathrm{~mL}$ of red wine (Flow rate of HPLC mobile phase: $0.7 \mathrm{~mL} / \mathrm{min}$ )

Fig. 4-1 Schematic diagram of special cell constructed for measurement of total surface area by pulsed amperometry, with the three electrodes connected to PalmSens electrochemical analyzer....... .55

Fig. 4-2 SEM images of a MIPPy/CNTs/QDs-modified stainless steel syringe needle tip.

Fig. 4-3 Effect of template OTA concentration in pre-polymerization mixture on $\mu$ SPE efficiency of MIPPy/CNTs/QDs-modified needle. .64

Fig. 4-4 Effect of OTA analyte concentration on recovery of automated MIPPy/CNTs/QDs- $\mu$ SPE-PE-HPLC-FD. .65 
Fig. 4-5 Standard calibration curve of automated MIPPy/CNTs/QDs- $\mu$ SPE-PE-HPLC-

FD. .66

Fig. 4-6 (a )Comparison of PAD currents measured for MIPPy/CNTs/QDs-modified needle, MIPPy-modified needle, and PPy-modified needle. .68

(b) Calibration of PAD current versus nominal surface area of commercially available stainless steel syringe needles of different gauges and lengths .68

Fig. 4-7 Recovery of OTA from $1 \mathrm{ng} / \mathrm{mL}$ standard solution by MIPPy/CNTs and MIPPy/CNTs/QDs-modified needles of different total surface areas.

Fig. 4-8 (a) Direct HPLC-FD analysis of the Italian red wine; (b) Direct HPLC-FD analysis of the Italian red wine spiked at $0.5 \mathrm{ppb}$ OTA; (c ) MIPPy/CNTs$\mu$ SPE-PE-HPLC-FD analysis after enrichment of OTA from $1.0 \mathrm{~mL}$ of the Italian red wine spiked at $0.5 \mathrm{ppb}$ OTA ;(d) MIPPy/CNTs/QDs- $\mu$ SPE-PEHPLC-FD analysis after enrichment of OTA from $1.0 \mathrm{~mL}$ of the Italian red wine spiked at $0.5 \mathrm{ppb}$ OTA

Fig. 4-9 Recoveries of red wind matrix components and OTA using MIPPy/CNTs- and MIPPy/CNTs/QDs- modified needles. Data from a non-imprinted $\mathrm{PPy} / \mathrm{CNTs} / \mathrm{QDs}-\mathrm{modified} \mathrm{needle} \mathrm{are} \mathrm{included} \mathrm{for} \mathrm{comparison}$ 73

Fig. 4-10 Evaluation of different solvents for their DPE effectiveness on OTA, by using $600 \mu \mathrm{L}$ of each solvent (in three times) for washing the MIPPy/CNTs/QDsmodified needle prior to PE-HPLC-FD analysis. (a) DDW/85\% $\mathrm{H}_{3} \mathrm{PO}_{4}(60: 1$ by volume); (b) $1.0 \mathrm{M} \mathrm{HCl}$

Fig. 4-11 (a) Direct HPLC-FD analysis of $0.5 \mathrm{ppb}$ spiked Italian red wine; (b) Direct HPLC-FD analysis of red wine spiked at 0.5 ppb OTA (red line); (c) 
MIPPy/CNTs/QDs- $\mu$ SPE-PE-HPLC-FD analysis after enrichment of OTA

from $1.0 \mathrm{~mL}$ of Italian red wine spiked at $0.5 \mathrm{ppb}$ OTA; (d)

MIPPy/CNTs/QDs- $\mu$ SPE-PE-HPLC-FD analysis after enrichment of OTA

from $1.0 \mathrm{~mL}$ of Canadian red wine spiked at $0.5 \mathrm{ppb}$ OTA...............................77

Fig. 4-12 Chemical structures of several phenolic compounds in red wine matrix...........78

Fig. 4-13 HPLC-FD analysis of differential pulsed elution (DPE) of red wine matrix components with $3 \times 200 \mu \mathrm{L}$ of $1.0 \mathrm{M} \mathrm{HCl}$, and final pulsed elution (FPE) of OTA with $1 \times 200 \mu \mathrm{L}$ of $2 \%$ TEA. (a) Italian red wine; (b) Canadian red wine .79

Fig. A-1 Diagram of AS-100 HRLC automatic sampling system.................................... 96

Fig. A-2 Fluid path in complete loop filling operation..................................................97

Fig. A-3 Flow chart of the complete filling method.......................................................98

Fig. A-4 Fluid path in partial loop filling method.......................................................99

Fig. A-5 Flow chart of the partial filling method........................................................99

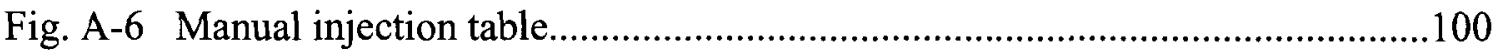

Fig. A-7 Flush table

Fig. A-8 Diagram of the Control panel key board .......................................................103

Fig. A-9 System configuration screen..........................................................................103

Fig. A-10 The sample sequence table............................................................................. 


\section{GLOSSARY}

\begin{tabular}{|l|l|}
\hline AFM & Atomic force microscope \\
\hline BET & Brunauer-Emmett-Teller \\
\hline CNTs & Carbon nanotubes \\
\hline DDW & Deionized distilled water \\
\hline DPE & Differential pulsed elution \\
\hline FD & Fluorescence detection \\
\hline HPLC & High performance liquid chromatography \\
\hline IAC & Immunoaffinity columns \\
\hline LOD & Detection limit \\
\hline LOQ & Quantification limit \\
\hline MIP & Molecularly imprinted polymer \\
\hline MIPPy & Molecularly imprinted polypyrrole \\
\hline MIT & Molecular imprinting technology \\
\hline MISPE & Molecularly imprinted solid phase extraction \\
\hline$\mu$ MISPE & Micro molecularly imprinted solid phase extraction \\
\hline MWNT & Multi-walled carbon nanotubes \\
\hline OTA & Ochratoxin A \\
\hline PAD & Pulsed amperometry detection \\
\hline PE & Pulse elution \\
\hline PPy & Polypyrrole \\
\hline Py & Pyrrole \\
\hline
\end{tabular}




\begin{tabular}{|l|l|}
\hline QDs & Quantum dots \\
\hline R.S.D. & Relative standard deviation \\
\hline SEM & Scanning electron microscopy \\
\hline SPE & Solid phase extraction \\
\hline$\mu$ SPE & Micro-SPE \\
\hline$\mu$ SPP & Micro solid phase preconcentration \\
\hline SSA & Specific surface areas \\
\hline SWNT & Single-walled carbon nanotubes \\
\hline TEA & Triethylamine \\
\hline TBAP & Tetrabutylammonium perchlorate \\
\hline
\end{tabular}




\section{LIST OF APPENDICES}

Appendix 1 Bio-Rad Model AS-100 HRLC Automatic Sampling System Operation

Manual .94

Appendix 2 Publications and Poster Presentations..........................................................111 


\title{
CHAPTER 1
}

\author{
Introduction
}

Reproduced with permission of the copyright owner. Further reproduction prohibited without permission. 


\subsection{Molecularly imprinted polymers}

To meet new challenges in environmental contamination, food safety and public health, analytical methods with high sensitivity and selectivity, and good and fast responses are in great need. Recent developments in the molecular imprinting technology (MIT) allow for the formation of specific recognition sites in synthetic polymers through the use of template or imprint molecules [1]. These recognition sites have a predetermined selectivity for analyte of interest and the resultant is known as a molecularly imprinted polymer (MIP). MIPs are synthesized by the co-polymerization of an appropriate monomer carrying certain functional groups and a cross-linker in the presence of the target analyte (the template molecules). The template molecules are "frozen" into positions by polymerization and the subsequent removal of the template leaves specific binding cavities. The cavities are complementary to the target compound in size, shape and in the arrangement of functional groups within the polymer network [2]. Ideally, MIPs are capable of rebinding the template in preference to other closely related structures [3]. The common principles of molecular imprinting are summarized in the scheme shown in Fig. 1-1 [4].

Since these man-made polymeric receptors possess a number of advantages over their biological counterparts (such as antibodies and enzymes) including low cost, storage stability, repeated operations without loss of activity, high mechanical strength, durability to heat and pressures, and applicability in harsh chemical media $[5,6]$, they have been widely used as affinity sorbents in chromatographic separations [7], and solid phase extraction (SPE) [8]. 


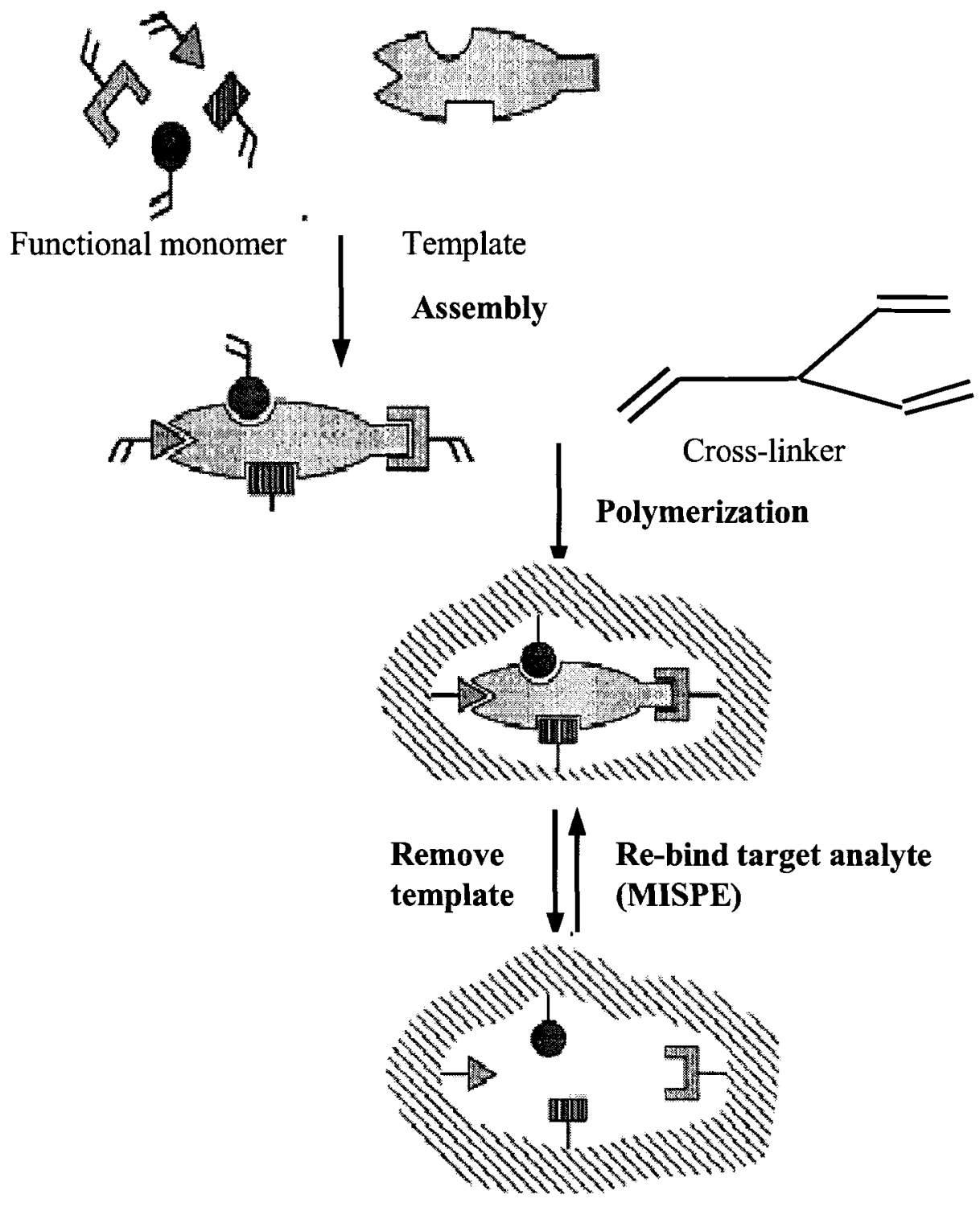

Fig. 1-1 Schematic principle of molecular imprinting

(The functional monomers are around the template as a result of the interactions between complementary chemical functionalities. After polymerization, the template is removed. The recognition site is exposed possessing a "memory" for the shape and chemical functionality of the imprint template. Adopted from reference 4) 


\subsection{Current state of knowledge}

\subsubsection{Development of molecular imprinting technology}

Although the start of molecular imprinting was marked in 1972 when Wulff [9] and Klotz [10] independently reported the preparation of organic polymers with predetermined ligand selectivities, some 40 papers describing conceptually similar approaches had appeared tracing back to the 1930s [11a]. Polyakov first reported some unusual adsorption properties in silica particles by pre-treating silicic acid with organic adsorbates before polycondensation in 1931[11b]. Later in 1949, Dickey created affinity for dye molecules in silica gel $[12,13]$. The methodology used by Dickey was very similar to that of Polyakov, but in this case methyl orange (and other alkyl orange dyes) was used as the template and the template was present from the start of the reaction process. Dickey's results demonstrated pronounced selectivity for 'pattern' dye which had been present during polymerization as related to the other dyes. However, the MIT research had been declined as the limitation of Dickey's silica samples was identified by Haldeman and Emmett in term of stability and reproducibility [14].

Modern molecular imprinting appeared with the introduction of covalent MIPs made by Wulff and Klotz in 1972. About 10 years later, Mosbach et al. presented a successful preparation of MIP using non-covalent approach [13,14]. These techniques offered a means of preparing molecular imprints in organic matrices and the limitations of the silica material were avoided. The interest in MIP has since then accelerated to yield an almost exponential increase in published papers during the past few years [14]. The explosive growth of research activity within the field of the design, development and application of MIPs over recent years reflects the maturation of this field and the broad interest it has attracted from 
the scientific community in general.

Nowadays, molecular imprinting has become a straightforward and versatile method for the generation of the tailor-made separation materials, antibody and receptor mimics in assay systems, biomimetic recognition elements in biological sensors, and artificial enzyme systems for catalytic applications.

\subsubsection{Theory of molecularly imprinted polymers}

High ligand selectivity and affinity are the characteristics of MIPs which have been observed and reported in great detail within past decades. Much of the literature available on the subject underlined the specific selectivity and affinity exhibited by MIPs with the substrate-selective mechanisms being analogous to that of natural entities such as antibodies and enzymes. It has been believed that concepts underpinning molecular imprinting theory are perhaps best exemplified by a theory originating in biochemistry [15]. Unfortunately, at the time of Polyakov's first paper, the origin of the selectivity of the antibodies in the immune system was under debate [13]. Two divergent concepts evolved: the selective theory and the instructional theory. The selective theory was first introduced in 1900 by Ehrlich while the instructional theory was originally suggested by Breinal and Haurowitz in 1930. These two theories have given way to the footprint (imprinting) mechanism which was presented by Haldeman and Emmett in 1955. After the reinvestigation of Dickey's silica samples, Haldeman and Emmett believed that in the process of methanol extraction, dye molecules on or near the surface were removed leaving micropores (footprints) with geometry and properties characteristic of the dye. The number of such pores should increase with the basicity of the dyes [11b, 13]. The footprint (imprint) mechanism was later challenged by Morrison et al. In 1959, the group of Morrison 
re-examined Dickey's silica sample. They instead proposed an association mechanism, in which 'trapped' template acted as nucleation sites, as the underlying basis for selectivity. They found that acid washing in Dickey's experiment did not lead to any significant extraction of the template. Morrison et al. concluded that the enhancement of specific adsorption following acid washing could not be explained by the removal of template molecules from footprints, but by an enhanced accessibility to remaining dye molecules, which constituted nucleation centers. In summary, although much of the data gave support to the footprint mechanism, the possibility that an association mechanism plays a role could never be conclusively overlooked [13].

Although the physical basis of molecular recognition is not entirely understood, some generally accepted basic guidelines of MIPs have been established. For example, it has been generally accepted that performance of MIP is a direct function of the nature and extent of the monomer-template interactions present in the pre-polymerization mixture [16]. The more stable or stronger the monomer-template complex, the more selective the MIP will be. This rule has been presumably used to select functional monomers [17]. Since the polymerization porogen plays an important role in the recognition properties of MIPs, selection of porogen is also a key factor for a successful MIP. It has been believed that MIPs synthesized with aprotic porogens usually have higher affinity and selectivity than those prepared using porogens with moderate or strong hydrogen bonding capacity [18]. It is also widely accepted that an ideal MIP is generally the result of good spatial orientation and intermolecular interactions $[16,17]$.

In summary, although significant improvements in understanding the molecular imprinting processes have been achieved and many new types of polymers, polymer 
formats and application areas have been investigated, a number of significant fundamental challenges regarding the mechanisms underlying the technique remain to be explored and many opportunities for applications are still to be exploited.

\subsubsection{Conventional preparation of molecularly imprinted polymers}

MIPs can be prepared according to a number of approaches that are different in the interactions the templates are linked to the functional monomers and subsequently to the polymeric binding sites. Two major interactions have been employed: one is non-covalent interaction such as hydrogen bonding, ionic bonds, hydrophobic interactions, and van der Waals forces, while the other one is covalent bonding $[12,19,20]$. In the covalent system, a template-monomer complex is formed through a reversible chemical bonding independent of polymer formation, such as ester, ketal, Schiff-base and so on [19]. After polymerization, these bonds were cleaved to liberate the template and subsequently re-established in order to selectively bind the target molecules $[7,12]$. Covalent imprinting strategy is very stable and selective. However, the number of functional groups to react with the templates in the imprint is limited and the re-binding is slower due to the necessary formation of the covalent bonds between templates and MIPs.

The non-covalent method was pioneered by Mosbach and co-workers and it is believed to have several advantages, such as easy preparation, faster rebinding kinetics and the broad selection of functional monomers and possible target molecules [21]. This approach usually utilizes only non-covalent interactions (such as hydrogen bonds, ionic interactions, hydrophobic interactions) for both the molecular imprinting process and the subsequent rebinding [21]. High affinity binding sites can be generated using the non-covalent imprinting strategy, however, the limitation is that the templates and 
monomers must form a sufficient number of non-covalent intermolecular interactions and the heterogeneous binding sites are usually generated due to the relatively weak interactions utilized [22].

More recently, a hybrid approach, combining the advantages of both covalent and non-covalent imprinting approaches has been developed by Whitcombe and coworkers [6, 21]. They utilized covalent interactions in the imprinting process, whereas non-covalent interactions were employed in the rebinding processes $[6,21]$.

\subsubsection{Molecularly imprinted polymers for solid phase Extraction}

A particularly promising application of MIPs is as selective sorbents in solid phase extraction (SPE) of analytes present in low concentrations or in complex matrices [8, 24]. The advantages of molecularly imprinted solid phase extraction (MISPE) compared to conventional SPE sorbents are increased selectivity, specificity and affinity [25]. Compared with other selective sorbents such as immunoaffinity materials, MIPs demonstrate very good thermal and chemical stability, repeated operations without loss of activity and high mechanical strength. The advantages of MISPE over other selective sorbents have made it one of the most important techniques for selective sample extraction, pre-concentration and purification in biological, pharmaceutical, and environmental fields $[3,5]$.

In the common protocols of MISPE, the MIP particles are packed into a disposable cartridge or a micro column between two frits. After conditioning, the sample is percolated through the MIP and a subsequent washing step allows the removal of the interfering compounds retained by non-specific interactions. The elution of the analyte is achieved by percolating a solvent able to disrupt the interactions between the polymeric matrix and the analyte molecules [26]. The experimental set-up of MISPE has varied between different 
modes of on-line and off-line [27].

\subsection{Statement of the problem}

Traditionally, MIPs are prepared as bulk polymer monoliths, which are subsequently ground in order to create a large surface area [28]. However, this treatment of MIPs inherited some drawbacks, such as the heterogeneous distribution of the binding sites and shape [28], intensive labor and potential exposure to hazardous particles when toxic molecules are imprinted. The intensive labor involves time-consuming grinding and sieving of a bulk MIP to obtain fine particles for the subsequent SPE applications [29]. Some studies have demonstrated that sieving of the particles can only produce about $20 \%$ of the original bulk polymer in particles of useful size [28]. To overcome the above problems, different synthetic strategies have been studied. Mostly, the attempts are focused on downsizing the bulk MIP materials into sub-micrometer and nanometer for the purpose of avoiding any ground and sieving step. However, packing MIP particles smaller than $10 \mu \mathrm{m}$ into a micro-column would cause high back pressure and the process is tedious [30].

Another limitation of bulk MIPs is that, depending on the synthesis procedure, there may be leaching of the template from the polymer, even after extensive washing, and thus contaminates the sample. This problem can be solved by using a structural analogue to the analytes of interest as the template for preparing a MIP. However, it is often difficult to find suitable analogues for practical application. Another possible solution is to use imprinted nano-materials which provide larger surface area and better accessibility to the template, hence, it facilitates a better removal of the original template [31].

The conventional approaches to prepare bulk MIPs have other disadvantages as well: the selective binding sites may be embedded in the polymer, and their ability to rebind 
analyte molecules of interest to the polymer structure is difficult [28].

All this explains that the development of alternative format of MIPs and new methodologies for production of MIPs are in great need.

\subsection{Methodology}

\subsubsection{Molecularly imprinted polypyrrole based nano-composites}

Nano-materials are gaining more and more attention because their small physical size offers much improved and even new functions that cannot be achieved with bulk materials. In this respect, nano-structures bearing tailor-made molecular binding properties are very attractive and they can potentially be used in different applications, such as affinity separations, substrate and enantioselective catalysis, and development of biomimetic sensors [32]. One challenge, however, is to devise ways in which the appropriate molecular recognition elements can be incorporated [33]. Among the different synthetic strategies that are presently studied, molecular imprinting is probably the mostly straightforward to produce nano-materials bearing predetermined selective binding properties [32]. In comparison with bigger particles or polymers prepared by bulk polymerization, imprinted nano-materials provide large surface area and monodispersity of size [31]. These properties facilitate a better removal of the original template, rapid diffusion and binding of the target molecules towards the imprinted polymer sites [31].

Among the polymeric matrices exploited to produce nano-composites with target molecule selectivity, acrylate-based, styrene-based, and silane-based polymeric materials are most frequently used [28, 31]. Currently, polypyrrole (PPy) and its derivatives began to play a leading role as a molecular recognition system due to its high environmental stability, electronic conductivity, ion exchange capacity, and biocompatibility [34]. Pyrrole (Py) is 
believed to be able to contribute $\mathrm{p}-\mathrm{p}$ interactions, electrostatic interactions, strong hydrogen bonding with a wide range of the template molecules [35] and it is also a versatile monomer to fabricate various hybrid nano-structures [36, 37 38]. Due to the superior properties of Py and PPy, the choice of Py as a functional monomer to prepare imprinted PPy based nano-composite is gaining more and more attention. Wallace and co-workers developed a glucose sensor based on an array of carbon nanotubes coated with PPy-glucose oxidase. The array constitutes a planar electrode with an extraordinary increase in surface area resulting from the polymer coated nanotubes [39]. More recently, a nanocomposite film of MIPPy and CNTs was coated on the pore surface of a stainless steel frit and the frit was used as a micro solid phase preconcentration ( $\mu$ SPP) device for the determination of ochratoxin A (OTA)
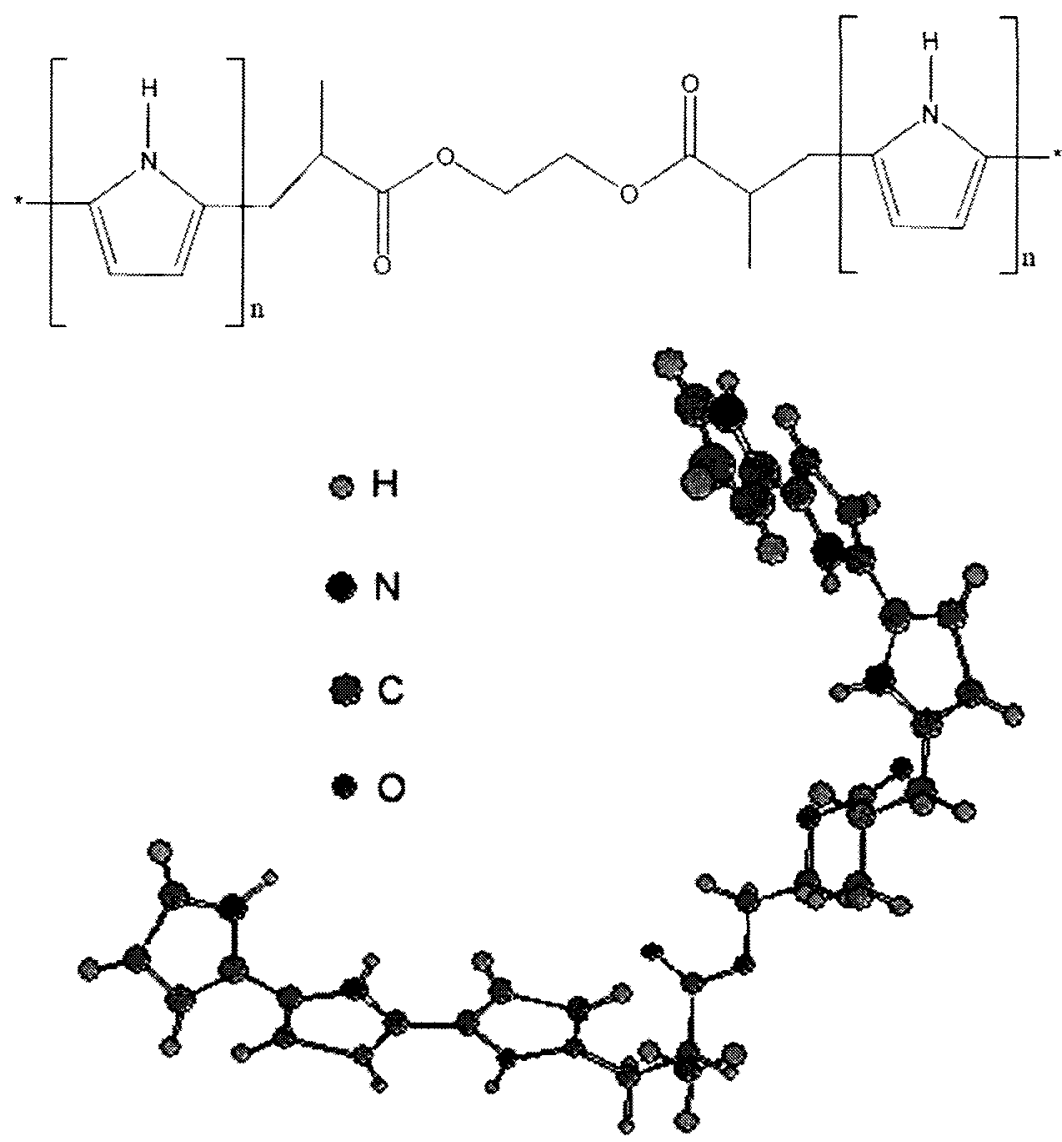

Fig. 1-2 A supposed model of PPy cross-linked by EDGMA [40] 
[30] and the highly cross-linked model of PPy with ethylene glycol dimethacrylate (EGDMA) was proposed as illustrated in Fig. 1-2 [40]. The achievements with MIPPy/CNTs on the frit opens new application opportunities for imprinted nano-composite, for example in SPE for selectively cleanup and preconcentration of compounds from a complex sample matrix [30].

\subsubsection{Electrochemical imprinting}

In general, the preparation of MIPs requires that functional and cross-linking monomers are copolymerized around the imprint species. Some preparation methods have been reported to produce imprinted polymer networks including suspension polymerization, modified surfaces, polymer colloids and miscellaneous techniques [41]. To produce MIPPy, three major methods have been applied based on induction of polymerization by different factors: (i) chemical initiation by oxidative agents; (ii) photo induced synthesis and (iii) electrochemical activation by anodic current (electropolymerization). Electropolymerization techniques have some attractive features including the easy preparation (by cycling potential sweeps from solutions), better adherence of the polymeric films to the surface of conducting electrodes of any shape and size with good reproducibility, and the ability to vary and control the polymerization conditions [42]. Moreover, this technique has been successfully exploited for the development of various types of PPy based nano-structural composites $[30,43,44,45,46]$. It is promising that the combination of electrochemical techniques with molecular imprinting opens new opportunities for development and application of new PPy based nano-structures.

\subsubsection{Pulsed elution and differential pulsed elution}

Pulse elution (PE) combined with MISPE was first introduced by Mullet and Lai in 
1998 [47]. This technology further evolved to differential pulsed elution (DPE). DPE involves intermediate wash steps to remove weakly bond structural analogues, prior to quantitative desorption of the strongly bound analyte [48]. MISPE-PE and MISPE-DPE allows for target analyte enrichment or pre-concentration by the injection of a relatively large volume of dilute sample solution, but a small volume of strong solvent or solution was used to elute the analyte of interest rapidly from the MISPE sorbent, thus further improving the detection limit. The other advantages of this technique are low consumption of solvents and fast analysis time [49].

MISPE-PE and MISPE-DPE can be coupled on-line with HPLC [40]. For determination of trace analytes, the integration of sample preparation and method of analysis into one set-up is very desirable.

\subsection{Objective}

Ochratoxin A (OTA) (Fig. 1-3), a group 2B carcinogen produced by Aspergillus and Penicillium fungi, has drawn considerable attention in bioanalytical chemistry [50]. Its widespread occurrence in different products including cereals, beans, nuts, coffee, cacao, beer, wine, milk and dried vine fruits is of concern to consumers/public health [51]. Recently, the analysis of OTA in red wine has been extensively reported in the literature because more and more studies revealed the presence of OTA in wine samples from African, American and European countries [52, 53, 54]. It is firmly believed to be an additional source of OTA intake for those countries in which wine is largely consumed $[53,55]$. The regulatory limits for OTA content in wine have been established in several countries, for example, the maximum allowable concentration of OTA in wine set by the European Union is $2.0 \mu \mathrm{g} / \mathrm{L}$ [55]. The legal limits for OTA in wines imply that the determination of OTA is 
therefore of great importance.

Due to the complexity of the red wine matrix, extraction/clean-up procedures are generally inevitable for OTA analysis. Extraction/clean-up can be carried out through solvent extraction, immunoaffinity columns (IAC) and SPE [56, 57]. Solvent extraction is usually avoided because they involve long analysis times and the use of harmful solvents [56]. Conventional SPE rely on relative unselective interaction, the resulting clean-up level may be insufficient for some challenge matrices [58]. The IACs exhibit unprecedented target selectivity, but unfortunately they are relatively costly and have a short shelf life [58, 59].

To address these problems, OTA-selective MIPs are considerably attractive as a potential alternative to immunoaffinity-based SPE. They are more robust and inexpensive, hence they can be a good candidate to develop affordable methods for the frequent screening of OTA contamination in foodstuff. The previous work in our group on stainless steel frits has demonstrated that nano-composite film of MIPPy and CNTs can be deposited on the pore surface of the frit to make $\mu$ MISPE device. The resultant frit exhibited a

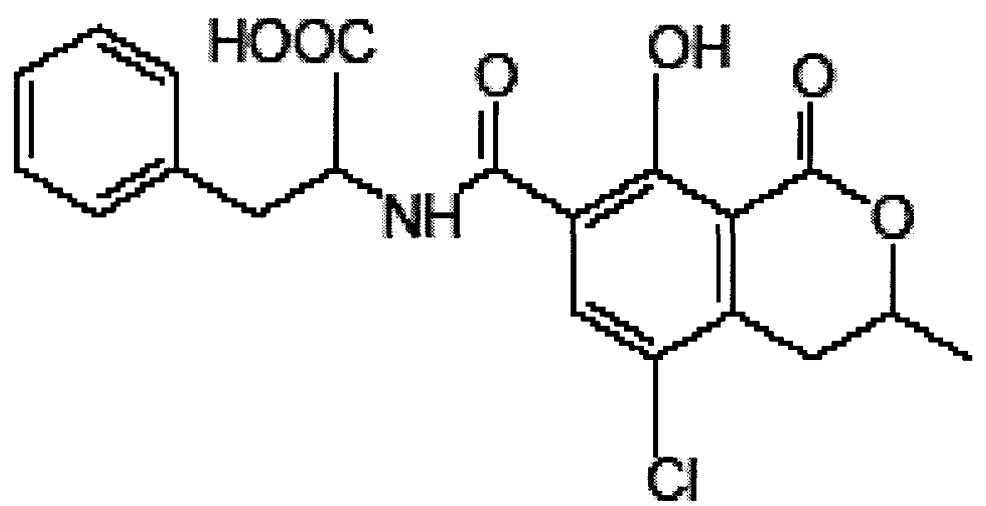

Fig. 1-3 Structure of OTA 
significant concentration capacity for trace level OTA in red wine.

The objective of this thesis research was to make a new $\mu$ MISPE device for the pre-concentration of trace amounts of OTA in red wine. The $\mu$ MISPE device can be utilized to modify a commercial HPLC autosampler, that allows full automation where sampling, extraction/clean-up, elution and injection are combined into a unique procedure. The automated procedure can be applied to real-world samples and it should provide shorter sample analysis time, more accurate quantification and better reproducibility and thus has good potential for routine analysis. The objectives were divided into two major aspects.

The first aspect was to synthesize OTA imprinted PPy based nano-composite which served as $\mu$ MISPE sorbents. The sorbents could be formed along the inner wall of a stainless steel syringe needle through in situ electropolymerization. The electropolymerization of the functional monomer (Py) and a crosslinker was also able to immobilize the OTA in the nano-composite of PPy (electrochemical imprinting). The electropolymerization conditions should be optimized to improve the performance of the needle.

Secondly, a fully automated procedure would be designed based on the idea of integration of sampling (extraction)/elution/injection (detection) and a new configuration of $\mu$ MISPE-PE-HPLC-FD could be built by coupling the $\mu$ MISPE modified needle to the autosampler. After removal of temple OTA, the resultant syringe needle would be employed both as $\mu$ MISPE device and interfacing device for a HPLC autosampler. To determinate trace level OTA, the modified syringe needle was first used as the sampling needle of the autosampler and the sample solution was able to pass through the needle. In this step, the sorbent packed inside the needle selectively extracts OTA from the sample 
solution. In the second step, the extracted OTA would be eluted from the sorbent by forcing a strong solvent through the needle at a faster flow rate and this eluate could be transferred to the sample loop of HPLC. If necessary, the DPE would be conducted to elute weakly bound components of the red wine matrix, then the final pulsed eluting of OTA would be performed in an usual way as PE. In the third step, the eluate could be automatically injected into the HPLC column and the quantification of OTA would be performed by fluorescence detection.

The experimental conditions in sample analysis should be optimized for effectively extracting OTA from the sample and completely eluting analyte OTA from the $\mu$ MISPE sorbent. The procedure should be simple and rapid in order to meet the requirement of routine analysis. 


\section{CHAPTER 2}

Experimental Section 


\subsection{Reagents and materials}

Ochratoxin A (OTA), pyrrole (Py), ethylene glycol dimethacrylate (EGDMA) and triethylamine (TEA) were purchased from Sigma-Aldrich (Mississauga, ON, Canada). Tetrabutylammonium perchlorate (TBAP) was obtained from Fluka (Buchs, Switzerland). Acetonitrile $\left(\mathrm{CH}_{3} \mathrm{CN}\right)$ and methanol $\left(\mathrm{CH}_{3} \mathrm{OH}\right)$ were HPLC-grade solvents obtained from Caledon (Georgetown, ON). Ammonium hydroxide and ammonium chloride were supplied by Fisher Chemicals (Fairlawn, New Jersey, USA). 18-M $\Omega \cdot \mathrm{cm}$ distilled deionized water (DDW) was obtained from a Millipore Milli-Q water system (Bedford, MD, USA).

Multi-walled carbon nanotubes (CNTs) with $\sim 95 \%$ purity were obtained from Shenzhen Nanotech Port (Shenzhen, China). These CNTs were prepared by catalytic decomposition of $\mathrm{CH}_{4}$, with diameters ranging from $10 \mathrm{~nm}$ to $30 \mathrm{~nm}$ and lengths ranging from $500 \mathrm{~nm}$ to $500 \mu \mathrm{m}$.

$\mathrm{CdTe} / \mathrm{CdS}$ sodium mercaptoproprionate quantum dots (ADS620QD) were supplied by American Dye Source (Baie D'Urfe, QC, Canada).

Bottles of Italian and Canadian red wines were purchased from a local store in Ottawa.

\subsection{Preparation of ochratoxin A standards}

A stock solution of $100 \mu \mathrm{g} / \mathrm{mL}$ OTA in methanol was prepared and the concentration was verified spectrophotometrically (M.W. $\left.=403.8, \varepsilon_{333 \mathrm{~nm}}=5500 \mathrm{M}^{-1} \mathrm{~cm}^{-1}\right)$ [60]. A stock solution of $1 \mu \mathrm{g} / \mathrm{mL}$ OTA was prepared from appropriate dilution of $100 \mu \mathrm{g} / \mathrm{mL}$ stock solution with methanol. The stock OTA solutions were stored at $-20^{\circ} \mathrm{C} .10 \mathrm{~mL}$ of $10.0 \mathrm{ppb}$ (or $\mathrm{ng} / \mathrm{mL}$ ) of OTA standard solution were prepared by evaporating $100 \mu \mathrm{L}$ of $1 \mu \mathrm{g} / \mathrm{mL}$ OTA stock solution under a stream of $\mathrm{N}_{2}$ and dissolving the residue in acidified DDW. The DDW was acidified with $1.0 \mathrm{M} \mathrm{HCl}(1 \%$ by volume, $\mathrm{pH}=2.5)$. Different concentrations of 
OTA standards were prepared from appropriate dilution of $10.0 \mathrm{ppb}$ OTA with acidified DDW (acidified with $1 \%$ by volume of $1.0 \mathrm{M} \mathrm{HCl}, \mathrm{pH}=2.5$ ). The standards were stored at $0{ }^{\circ} \mathrm{C}$.

\subsection{High performance liquid chromatograph}

The HPLC set-up consisted of a solvent pump (Shimadzu LC-6A, Kyoto, Japan), an injector valve (Valco Cheminert VIGI C2XL, Houston, TX, USA) equipped with a $20-\mu \mathrm{L}$ sample loop, a column (Phenomenex polymerX RP-1, pH stability 0-14, $5 \mu \mathrm{m}, 250 \times 4.6$ mm, Torrance, CA, USA), a fluorescence detector (Water 470, Boston, MA, USA), an integrator (Spectra Physics 42900, San Jose, CA, USA) and a data acquisition system (PeakSimple, Torrance,CA, USA).

For HPLC, 20:80 v/v acetonitrile-ammonia buffer $\left(\mathrm{NH}_{4} \mathrm{Cl} / \mathrm{NH}_{3}, 20 \mathrm{mM}, \mathrm{pH}=9.2\right)$ was used as the mobile phase at a flow rate of $1.0 \mathrm{~mL} / \mathrm{min}$ or $0.7 \mathrm{~mL} / \mathrm{min}$. The excitation and emission wavelengths for fluorescence detection were set at $380 \mathrm{~nm}$ and $440 \mathrm{~nm}$, respectively. The optimization of HPLC-FD analysis was based on Yu et al's work [61].

\subsection{In-situ electropolymerization}

A platinum wire was used as the counter electrode and a $\mathrm{Ag} / \mathrm{AgCl}$ electrode was used as the reference electrode. A Stoneheart BC 1200 high-performance potentiostat (Madison, CT, USA) was used to apply a constant potential of $+0.85 \mathrm{~V}$ on a stainless steel syringe needle (BD, Franklin Lakes, New Jersey, USA). A syringe (BD 3-mL) filled with pre-electropolymerization mixture was connected with the syringe needle. It supplied 0.2 $\mathrm{mL}$ of pre-electropolymerization mixture to the needle every 10 minutes after the start of electropolymerization reaction. The applied potential was switched off after 30-90 minutes of polymerization. The set-up for in-situ electropolymerization is shown in Fig. 2-1. 


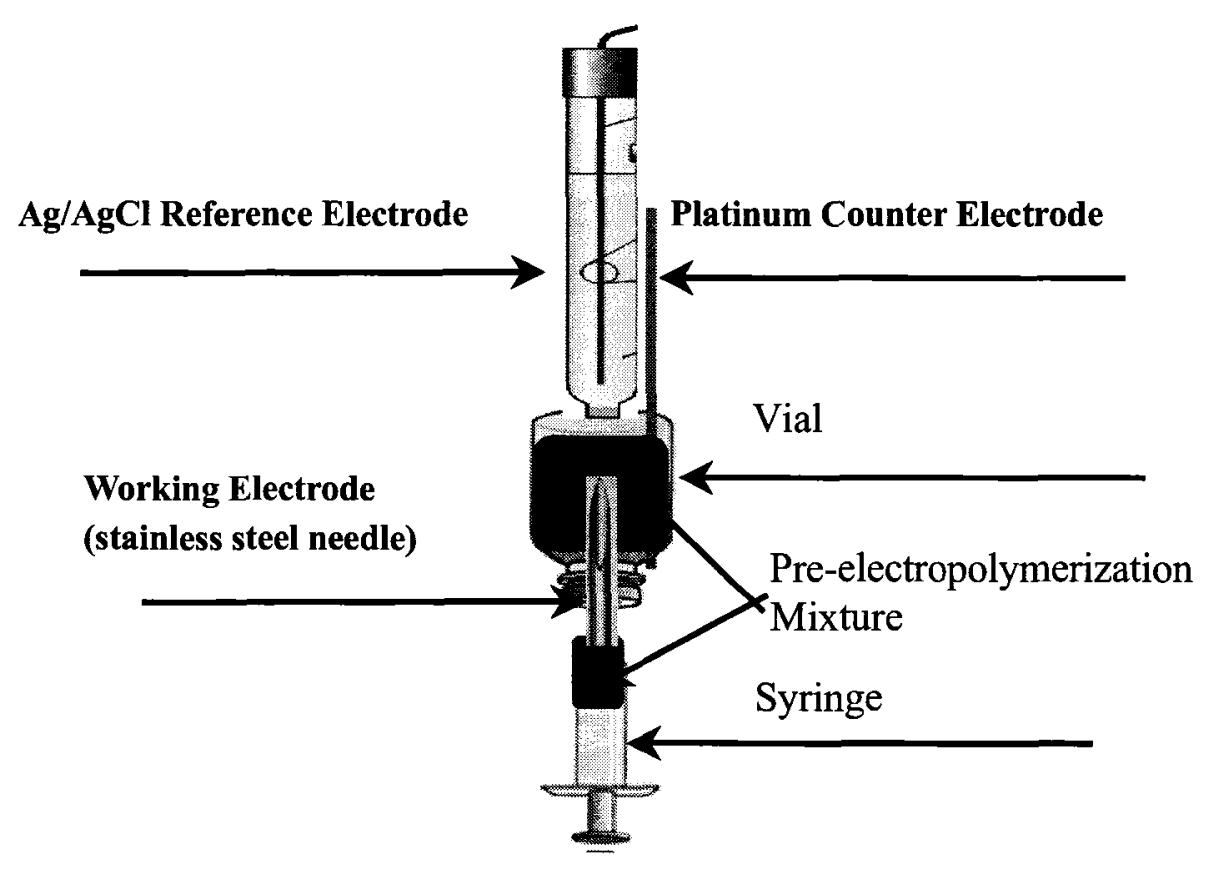

Fig. 2-1 Preparation of MIPPy/CNTs-modified needle by electropolymerization

\subsection{Removal of the template OTA from the modified needle}

The as-prepared syringe needle was connected with a $10 \mathrm{~mL}$ syringe $(\mathrm{BD}$, Franklin Lakes, New Jersey, USA) mounted on a syringe pump (KD Scientific 100, New Hope, PA, USA). The OTA template was removed by continually pumping eluting solvent through the needle and the eluates were analyzed with HPLC-FD. The washing continued until no OTA breakthrough peak was observed in the HPLC-FD analysis of eluate. The modified needle was finally washed with $200 \mu \mathrm{L}$ of DDW for surface re-conditioning.

\subsection{Backpressure test}

After the removal of the template, the modified stainless steel needle was assembled with a $3 \mathrm{~mL}$ syringe (BD, Franklin Lakes, New Jersey, USA) and mounted on the syringe 
pump. A $1.0 \mathrm{~mL}$ DDW was pumped through the needle at different flow rates between 0.10 $\mathrm{mL} / \mathrm{min}$ and $1.5 \mathrm{~mL} / \mathrm{min}$. The pump stopped automatically if the backpressure was too high.

\subsection{Off-line MIPPy/CNTs- $\mu$ SPE-PE-HPLC-FD analysis}

$0.5 \mathrm{~mL}$ of OTA standard solution or red wine was pumped through the MIPPy/CNTs-modified needle at a flow rate of $0.1 \mathrm{~mL} / \mathrm{min}$. All samples were acidified with $1.0 \mathrm{M} \mathrm{HCl}(1 \%$ by volume). After washing with $200 \mu \mathrm{L}$ of DDW to eliminate $\mathrm{HCl}$, the pre-concentrated OTA was pulse eluted with $50 \mu \mathrm{L}$ of $2 \%$ TEA (v/v) in HPLC mobile phase each time. The eluate was collected with a HPLC injection syringe and then immediately injected to the HPLC-FD for chromatographic analysis and the peak area was recorded. The MIPPy/CNTs- modified syringe needle was finally washed with $200 \mu \mathrm{L}$ of DDW for surface re-conditioning.

\subsection{Autosampler-HPLC system}

The HPLC instrument was the same as described in Section 2.2. A Bio-Rad (Hercules, CA, USA) AS-100 HRLC automatic sampler was added to build an automated HPLC-FD and $\mu$ MISPE-PE-HPLC-FD analysis system. The sampler was equipped with a six-port injection valve, a $20-\mu \mathrm{L}$ injection loop, and a tray of up to one hundred $1.5-\mathrm{mL}$ sample vials. In order to modify the commercial autosampler into an automated extraction system, the original injection needle was replaced by a $\mu$ MISPE sorbent packed needle. A short length of Nalgene connection tubing was used to mount up the $\mu$ MISPE sorbent packed needle (as shown in Fig. 2-2). It was necessary to equip the connection tubing with a septum that helped avoid sample leaking as well as minimize band broadening during the transfer of sample solution from the vial to the injection loop. 
Several parameters of the autosampler were set specifically to meet different demands and the autosampler was programmed to complete the integrated sampling (extraction)/pulsed elution (differential pulsed elution)/injection (detection) process. A detailed introduction about the components, parameters, functions, and operation procedures (including direct HPLC-FD analysis and automated $\mu$ MISPE-PE-HPLC-FD analysis) were described in the appendix 1 (The operation manual of Bio-Rad AS-100 HRLC automatic sampler).

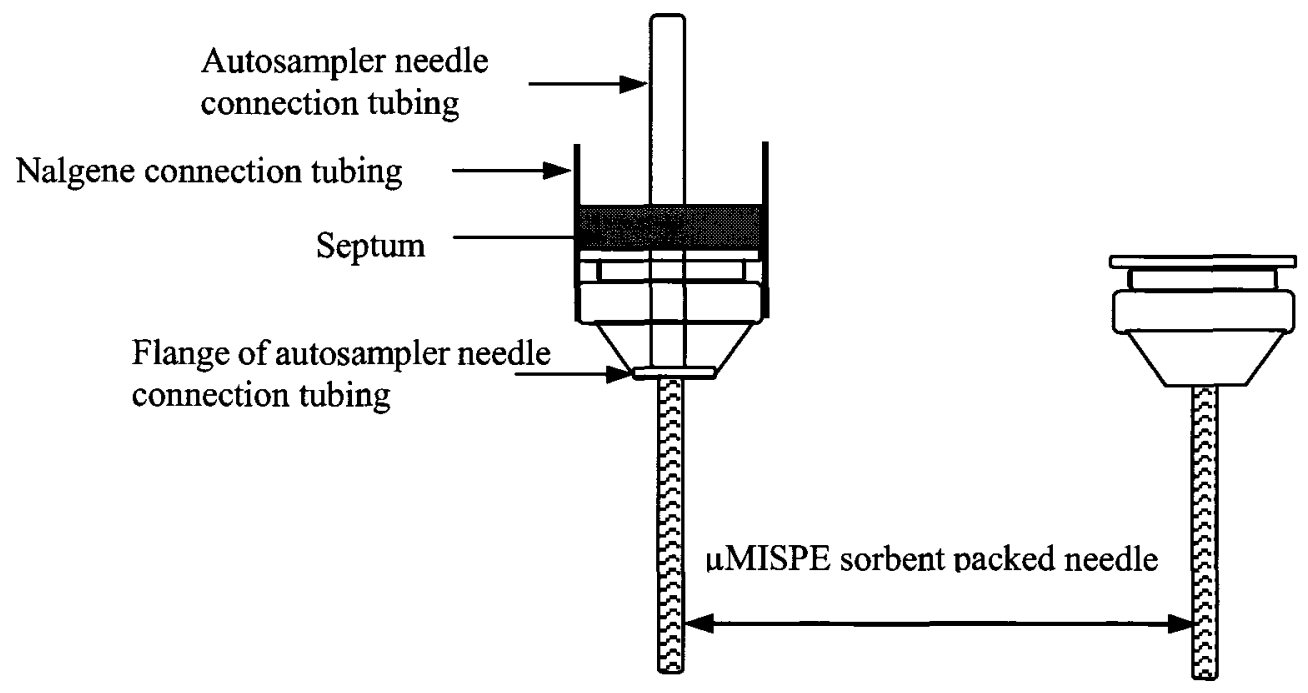

Fig. 2-2 Schematic diagram of connection of $\mu$ MISPE sorbent packed needle with the autosampler

\subsection{Automated $\mu$ MISPE-PE/DPE-HPLC-FD analysis}

The automated $\mu$ MISPE-PE/DPE-HPLC-FD analysis was based on a concept of in-needle molecularly imprinted extraction (as illustrated in Fig. 2-3), in which a $\mu$ MISPE sorbent packed needle replaced the original injection needle. Since the injection needle was specially modified with a $\mu$ MISPE sorbent, the analyte of interest could be selectively extracted from the sample solution and adsorbed on the $\mu$ MISPE sorbent as the liquid 
sample passing through the modified needle. In the off-line MIPPy/CNTs- $\mu$ SPE-PE-HPLC-FD analysis, the extraction step was performed by a syringe pump, while in the automated configuration it was conducted via the autosampler.

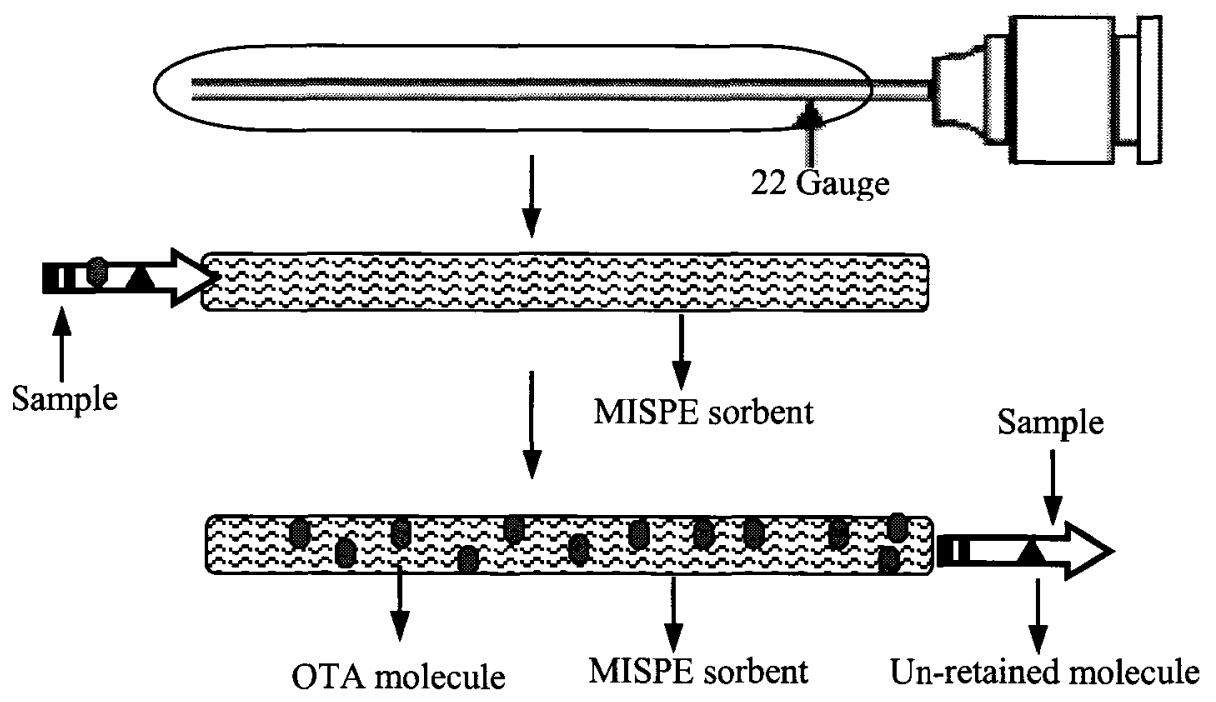

Fig. 2-3 Schematic diagram of in-needle $\mu$ MISPE

The automated $\mu$ MISPE-PE/DPE-HPLC-FD analysis is composed of three major steps. They are sampling (extraction), pulsed elution (or differential pulsed elution), and eluate injection (detection). The autosampler's programs for each step and parameters were described in appendix-the operation manual of Bio-Rad AS-100 HRLC automatic sampler.

\subsubsection{Sampling (extraction)}

Before sampling, $1.0 \mathrm{~mL}$ of DDW was uptaken to pre-condition the MIPPy/CNTs, MIPPy/CNTs/QDs-modified needle. Then a sample of $1.0 \mathrm{~mL}$ OTA standard solution (at different concentrations, acidified with $1.0 \mathrm{M} \mathrm{HCl}$ ) was uptaken from a sample vial at a flow rate of $0.1 \mathrm{~mL} / \mathrm{min}$. During the sample uptaking step, the six-port injection valve was in the LOAD position and HPLC mobile phase was pumped through the HPLC column by 
bypassing the autosampler loop. At this step, the OTA molecules were able to be extracted from the liquid sample and adsorbed on the MISPE sorbent inside the injection needle and the un-retained sample components were loaded on the autosampler loop (as illustrated in Fig. 2-4 (a).

Then, the sample loop and column were washed by the HPLC mobile phase (as illustrated in Fig. 2-4(b) until no OTA peak was observed by the FD in order to eliminate any possible carryover from the uptaking of sample solution.

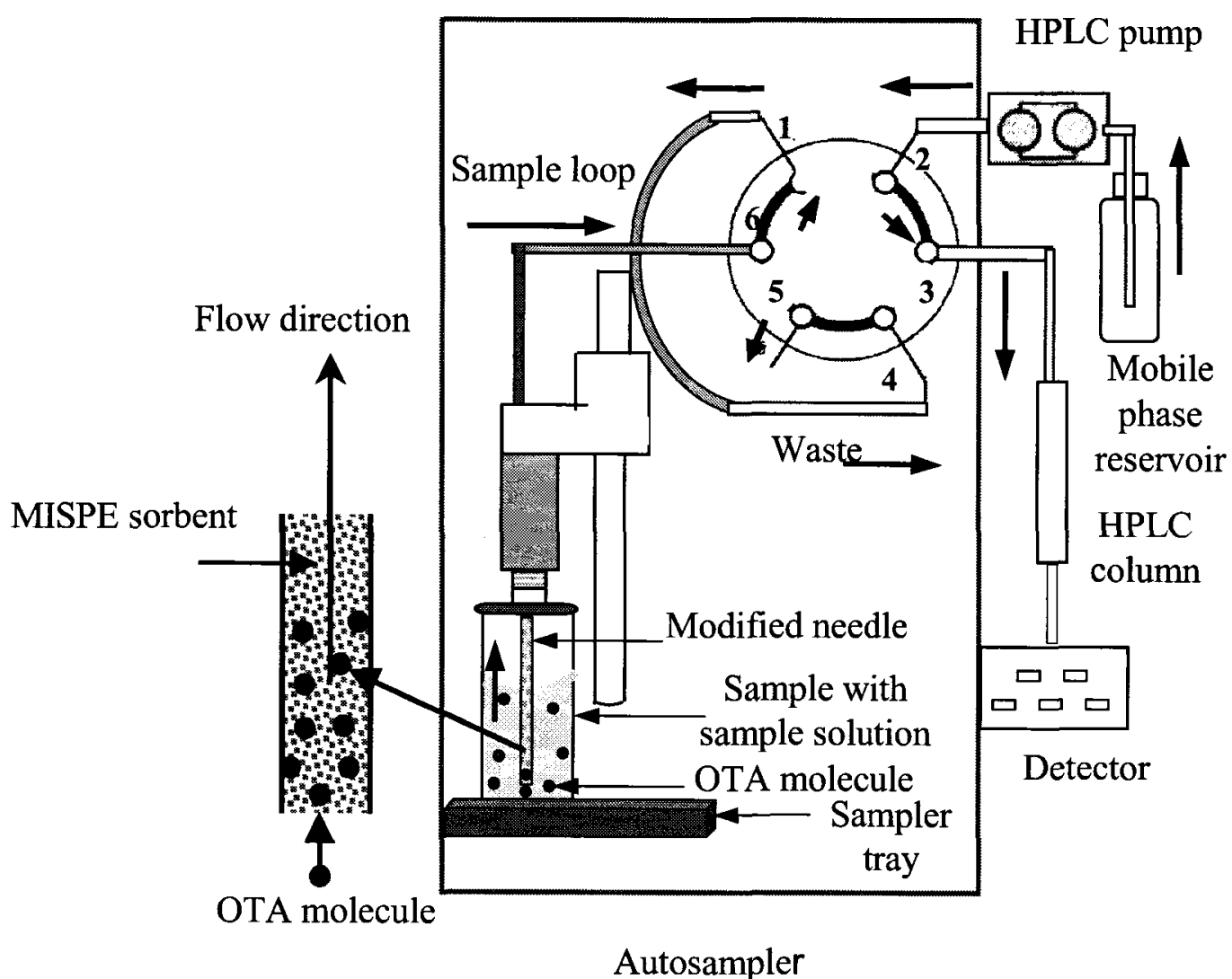

Fig. 2-4(a) Schematic diagram of automated $\mu$ MISPE-PE/DPE-HPLC-FD analysis in step 1-sampling (extraction). The modified needle is uptaking $1.0 \mathrm{~mL}$ of sample from vial 


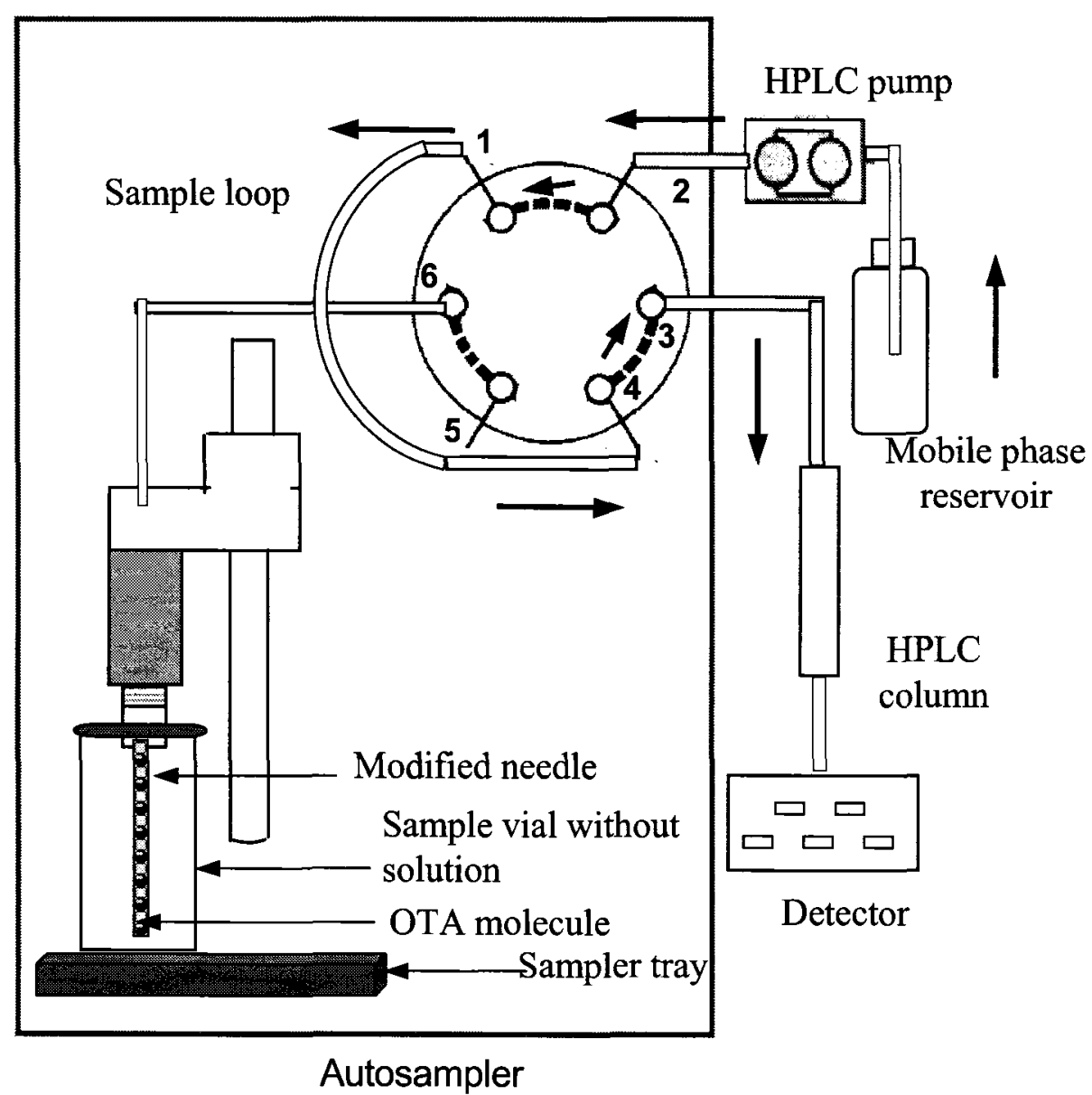

Fig. 2-4(b) Schematic diagram of automated $\mu$ MISPE-PE/DPE-HPLC-FD analysis in step 1-sampling (extraction). Sample loop and column are being washed with HPLC mobile phase

\subsubsection{Pulsed elution/Differential pulsed elution}

After washing the $\mu \mathrm{MISPE}$ sorbent packed needle with $200 \mu \mathrm{L}$ of DDW, the pre-concentrated OTA was pulse eluted by uptaking $200 \mu \mathrm{L}$ of $2 \%$ TEA (solution in HPLC mobile phase) from a sample vial at a flow rate of $1.0 \mathrm{~mL} / \mathrm{min}$ while the six-port injection valve was in the LOAD position (as illustrated in Fig. 2-5). In this step, the eluate was collected with the automated injection valve of autosampler and loaded into the sample loop of the autosampler. 


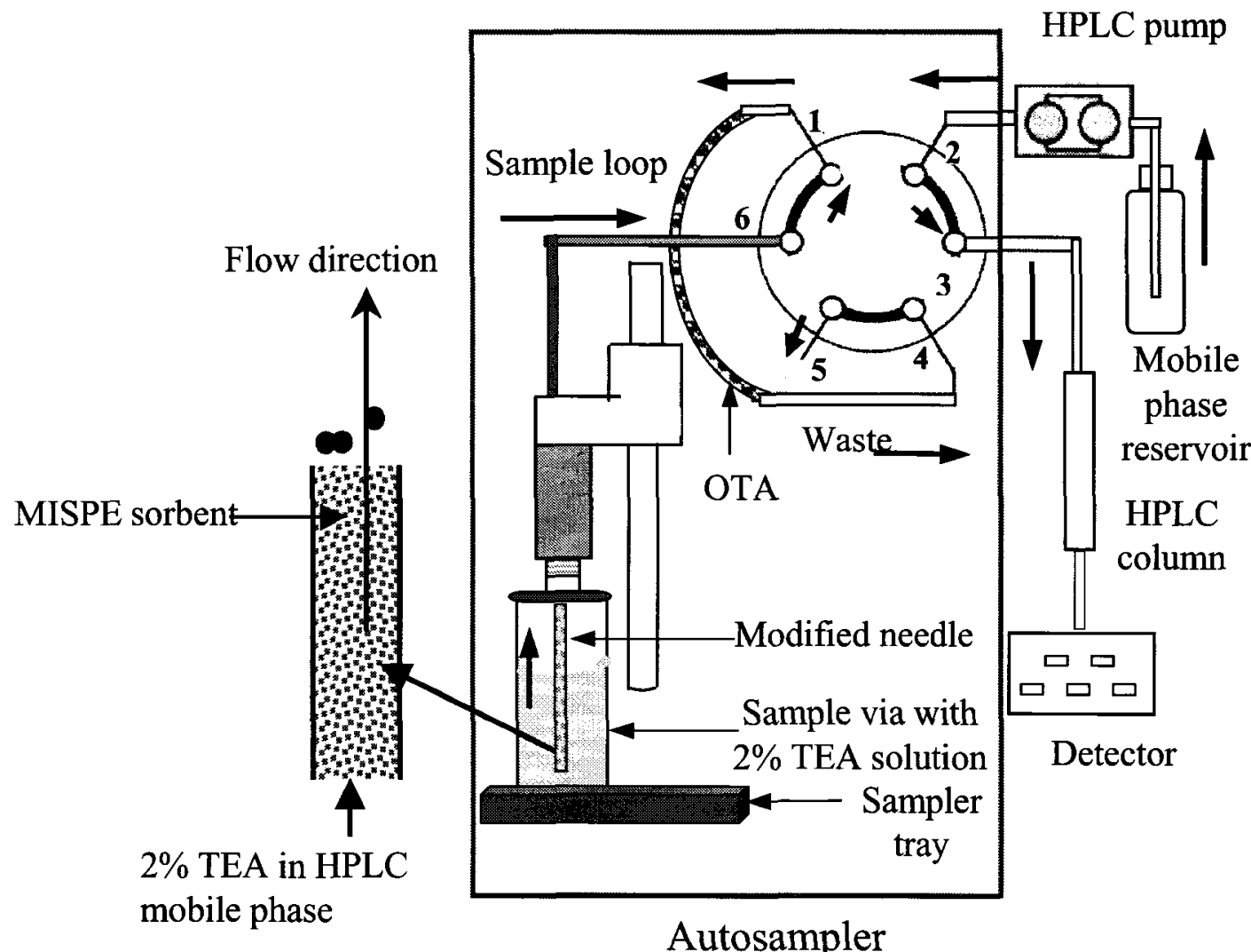

Fig. 2-5 Schematic diagram of automated $\mu$ MISPE-PE/DPE-HPLC-FD analysis in step 2-pulsed elution. The extracted OTA is being washed off the syringe needle with $2 \%$ TEA (prepared in HPLC mobile phase) and the eluate is collected with the automated injection valve of the autosampler system and loaded onto the sample loop.

In DPE process, $200 \mu \mathrm{L}$ of the DPE eluting solvent was employed each time to elute the weakly bound red wine matrix components prior final pulsed elution (FPE) of OTA. Each eluate of DPE was analyzed by HPLC-FD at a HPLC mobile phase flow rate of 1.0 $\mathrm{mL} / \mathrm{min}$. The DPE continued until no matrix peak was observed by the FD. Then the final pulsed elution (FPE) was conducted by using 2\% TEA in HPLC mobile phase as FPE solvent. The eluate of FPE was collected with the automated injection valve of autosampler 
and loaded into the sample loop of the autosampler.

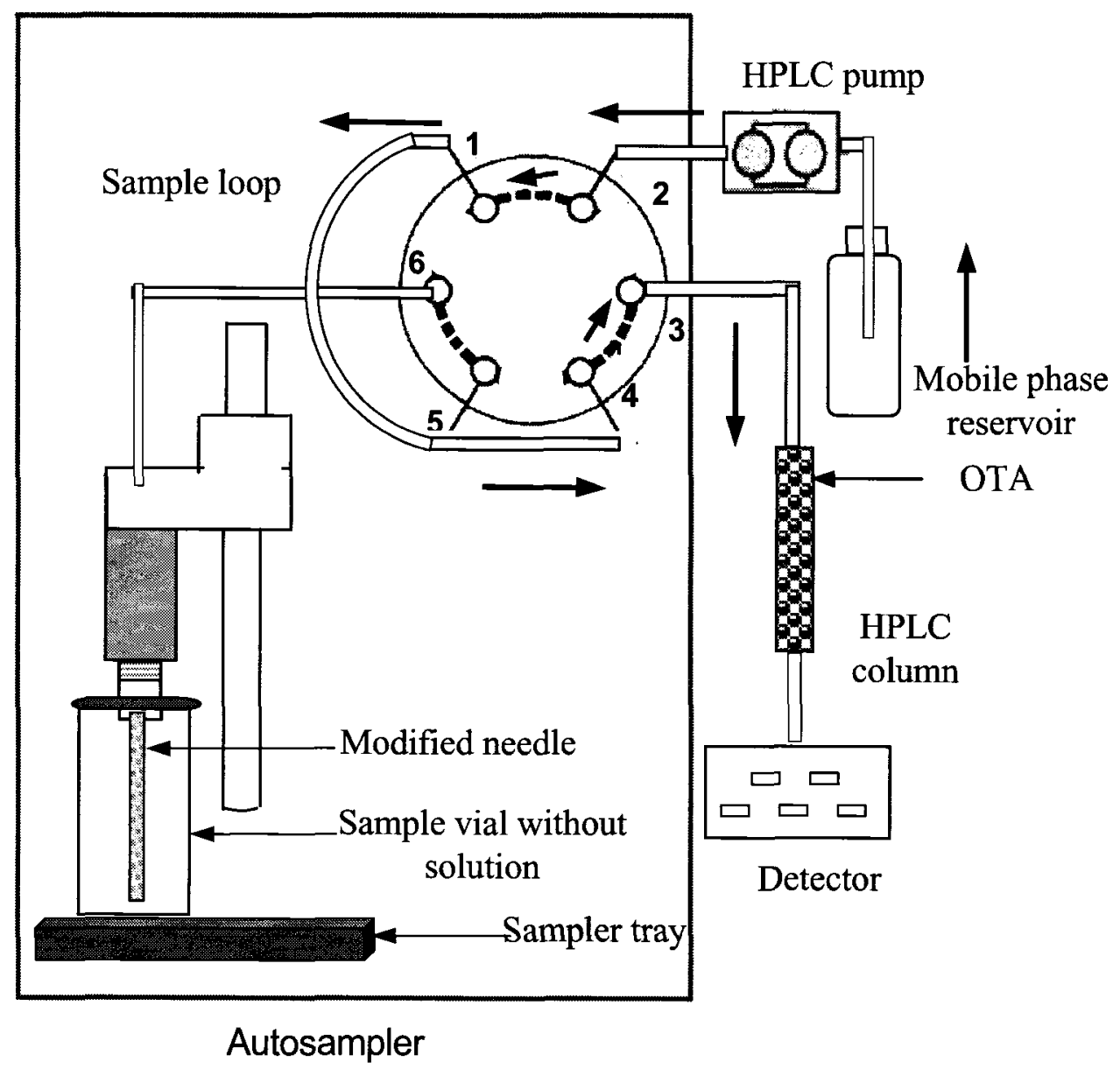

Fig. 2-6 Schematic diagram of automated $\mu$ MISPE-PE/DPE-HPLC-FD analysis in step 3-injection/detection. The eluate is injected onto the column from the sample loop. The sample loop and column are being washed with HPLC mobile phase

\subsubsection{Injection/detection}

The last step was to inject the $2 \%$ TEA eluate loaded onto the sample loop (described in 2.9.2) into the HPLC column and the extracted OTA was analyzed by HPLC-FD. The HPLC-FD was described in section 2.3 with a HPLC mobile phase flow rate of $1.0 \mathrm{~mL} / \mathrm{min}$. 
The HPLC-FD peak area for OTA was recorded.

\section{10 Calculation of recovery}

The percentage recovery by $\mathrm{PPy} / \mathrm{CNTs}$, MIPPy/CNTs and MIPPy/CNTs/QDs, modified syringe needle was calculated from OTA peak areas measured in $\mu$ MISPE-PE-HPLC-FD analysis versus direct HPLC analysis of the same 1.0-ppb OTA standard solution. After each $\mu$ MISPE-PE-HPLC-FD analysis, 2\% TEA (prepared in HPLC mobile phase) was used in a $200-\mu \mathrm{L}$ aliquot for the regeneration of modified syringe needle. The $\mu$ MISPE sorbent packed needle was finally washed with $100 \mu \mathrm{L}$ of DDW for surface re-conditioning.

\subsection{Scanning electron microscopy images}

Scanning electron microscopy (SEM) images were obtained by using JSM-5600LV SEM (JEOL, Japan). The MIPPy/CNTs and MIPPy/CNTs/QDs modified needles were adhered onto the SEM stage by a double-sided carbon tape for examination without carbon or gold sputtering. The SEM images were taken at an accelerating voltage of 5 to $10 \mathrm{kV}$. 


\section{CHAPTER 3}

Molecularly Imprinted Solid Phase Extraction in a Syringe Needle packed with Polypyrrole-encapsulated Carbon Nanotubes for Determination of Ochratoxin A in Red Wine 


\subsection{Introduction}

Today, more and more research has demonstrated that design and fabrication of advanced materials coupled with good understanding of their behaviors have profound impact on developments in analytical chemistry, and may hold the key to improvement of existing or new techniques at present times and near future [62]. With the introduction of new sorbents, molecularly imprinted SPE (MISPE) has shown the capability of trapping a wide variety of analytes. The application of these synthetic polymers as sorbents allows not only pre-concentration and cleaning of the sample but also selective extraction of the target analyte, which is important, particularly when the sample is complex and impurities can interfere with quantification [5].

Carbon nanotubes (CNTs) represent a new class of advanced materials, which have found applications in various fields of analytical chemistry as sensors, separation devices and SPE sorbents $[63,64,65]$. However, the potential application of CNTs to MISPE has been very little explored. CNTs are hollow graphitic nano-materials comprising one (single-walled carbon nanotubes, SWNT) or multiple (multi-walled carbon nanotubes, MWNT) layers of graphene sheets. The lengths of the nanotubes can range from several hundred nanometers to several micrometers with diameters ranging from 0.2 to $2 \mathrm{~nm}$ for SWNT and from 2 to $100 \mathrm{~nm}$ for coaxial MWNT. CNTs are novel and interesting nano-materials because of their high surface areas, good electrical and chemical properties [66].

Conducing polymers represent another excellent tool for the preparation of nano-composites with entrapped molecules and they have been used as a molecular recognition matrix through doping of anionic recognition elements [67]. PPy is one of the 
most extensively used conducting polymers and it has been combined with MIT to make sensors for a couple of analytes, such as serotonin, 1-naphthalenesulfonate, amino acids, caffeine, atropine, saccharide and glycoproteins [42].

The exceptional properties of CNTs and PPy have motivated researchers to develop and investigate nanotube-based PPy composite with tailored properties [68]. Cui et al electrodeposited PPy thin films on carbon nanotube (CNT) backbones. It was found that the presence of the CNT backbone greatly improved the anion exchange capacity and stability of the PPy-CNT composite film, which may be attributed to the high surface area of CNT matrix and nanostructure of the PPy film [38]. A biosensing electrode for electrochemical impedance spectroscopy for the measurement of DNA hybridization was developed by the doping of nucleic acid probes within a PPy film onto carboxylic group-functionalized MWNTs [69]. The composite film of MIPPy and CNTs on the pore surfaces of a stainless steel frit demonstrated significant recognition of OTA [30]. It was believed that binding capacity of MIPPy/CNT-modified stainless steel frits were enhanced because of ultra-high surface-to-volume ratio and nano-structured surface morphology [30].

In this chapter, we fabricated a new micro molecularly imprinted solid phase extraction ( $\mu$ MISPE) device. The new $\mu$ MISPE device reported here was designed for the pre-concentration of trace amounts of OTA in red wine samples. It was made by growing a three dimensional network of nano-composite of PPy and CNTs inside a 22-gauge stainless steel syringe needle. CNTs were encapsulated with OTA-imprinted PPy and the encapsulated CNTs served as the backbone of the three dimensional network to keep MIPPy/CNTs inside the syringe needle. In comparison to the conventional (and commercially available) SPE, the MIPPy/CNTs sorbent is in-situ electropolymerized [70], 
thus, there is no need for a separate procedure to pack the $\mu$ MISPE sorbent inside the needle.

\subsection{Experimental}

\subsubsection{Optimization of the in-situ electropolymerization time}

Three different electropolymerization times (45 $\mathrm{min}, 60 \mathrm{~min}$ and $90 \mathrm{~min}$ ) were employed to make MIPPy/CNTs-modified stainless steel syringe needles. About $100 \mu \mathrm{M}$ OTA and $1.0 \%$ by weight of CNTs were present in the pre-polymerization mixture when Py was electropolymerized within each 22-gauge stainless steel syringe needle (BD 22G, Franklin Lakes, New Jersey, USA). The concentrations of Py, TBAP, and EGDMA were $0.1 \mathrm{M}, 0.1 \mathrm{M}$ and $0.2 \mathrm{M}$ respectively in the pre-polymerization mixture with acetonitrile as the porogen. For electrochemical preparation of MIPPy-encapsulated CNTs, the needle was filled with the pre-polymerization mixture and then connected to serve as the working electrode. The set-up for electropolymerization is shown in Fig. 2-1.

After the electropolymerization, the template OTA was removed. The effect of the electropolymerization time was evaluated in terms of the backpressure the needles exhibited (for backpressure test see section 2.6).

\subsubsection{Choose of the eluting solvent}

$\mathrm{MeOH}, \mathrm{MeCN}, 1 \%$ TEA in HPLC mobile phase and $2 \%$ TEA in HPLC mobile phase were used to investigate their efficiency for removal of OTA template from those needles electropolymerized for 60 minutes. The off-line washing procedure was described in section 2.5. The amount of OTA eluted from the needle was calculated based on the mass of the eluate and OTA concentration found in the eluate.

\subsubsection{Effect of sample pH on MIPPy/CNTs- $\mu$ SPE-PE-HPLC-FD}


A standard $10.0 \mathrm{ppb}$ (or $\mathrm{ng} / \mathrm{mL}$ ) OTA solution was prepared by evaporating the appropriate volume of a $1 \mu \mathrm{g} / \mathrm{mL}$ OTA stock solution in methanol under a stream of $\mathrm{N}_{2}$ and dissolving the residue in acidified DDW with different $\mathrm{pH}$ values $(\mathrm{pH}=1.5,2.5,4.0)$. The DDW was acidified with $1.0 \mathrm{M} \mathrm{HCl}$ and the $\mathrm{pH}$ of the acidified DDW was measured by a $\mathrm{pH}$ meter (Accumet $\mathrm{pH}$ meter Model 140, Fisher). A $1.0 \mathrm{ppb}$ (or $\mathrm{ng} / \mathrm{mL}$ ) OTA standard solution was prepared by the dilution of the $10.0 \mathrm{ppb}$ (or $\mathrm{ng} / \mathrm{mL}$ ) OTA standard solution with the corresponding acidified DDW $(\mathrm{pH}=1.5,2.5$ and 4.0).

The Off-line MIPPy/CNTs- $\mu$ SPE-PE-HPLC-FD analysis (described in section 2.5) was performed by using the above prepared $1.0 \mathrm{ppb}$ (or $\mathrm{ng} / \mathrm{mL}$ ) OTA standard solutions as working solutions and the needle electropolymerized for 60 minutes as the $\mu$ MISPE device. A plot of MIPPy/CNTs- $\mu$ SPE-HPLC-FD peak area vs sample $\mathrm{pH}$ was obtained.

\subsubsection{Effect of preconcentration flow rate on MIPPy/CNT- $\mu$ SPE-PE-HPLC-FD}

All red wine samples were filtrated with $0.45-\mu \mathrm{m}$ pore size filter (Nylon ID $13 \mathrm{~mm}$, ExpediGlass) and acidified with $1.0 \mathrm{M} \mathrm{HCl}$ ( $1 \%$ by volume). A $0.5 \mathrm{~mL}$ sample of spiked red wine (containing $0.5 \mathrm{ng} / \mathrm{mL}$ OTA) was pumped through the MIPPy/CNTs-modified syringe needle electropolymerized for 60 minutes at a different flow rate over the range of $0.05 \mathrm{~mL} / \mathrm{min}$ to $0.50 \mathrm{~mL} / \mathrm{min}$. The offline MIPPy/CNT- $\mu$ SPE-PE-HPLC-FD analysis (described in section 2.7) was conducted. A plot of MIPPy/CNTs- $\mu$ SPE-HPLC-FD peak area vs preconcentration flow rate was obtained.

\subsubsection{Standard calibration curve}

All red wine samples were filtrated with $0.45-\mu \mathrm{m}$ pore size filter (Nylon ID $13 \mathrm{~mm}$, ExpediGlass) and acidified with $1.0 \mathrm{M} \mathrm{HCl}$ ( $1 \%$ by volume). The offline MIPPy/CNT- $\mu$ SPE-PE-HPLC-FD analysis (described in section 2.7) was conducted by 
using spiked red wine (containing OTA ranging from 0.1 to $0.8 \mathrm{ng} / \mathrm{mL}$ ) as working solution and the needle electropolymerized for 60 minutes as the $\mu$ MISPE device. A plot of MIPPy/CNTs- $\mu$ SPE-PE-HPLC-FD peak area versus OTA concentration was obtained.

\subsubsection{Application of MIPPy/CNTs- $\mu$ SPE-HPLC-FD to red wine analysis}

The Italian red wine sample was filtrated with $0.45-\mu \mathrm{m}$ pore size filter (Nylon ID 13 $\mathrm{mm}$, ExpediGlass) and acidified with $1.0 \mathrm{M} \mathrm{HCl}$ ( $1 \%$ by volume). $0.5 \mathrm{~mL}$ of red wine sample and spiked red wine sample (containing $0.5 \mathrm{ng} / \mathrm{mL}$ OTA) were analyzed by offline MIPPy/CNT- $\mu$ SPE-PE-HPLC-FD (described in section 2.7). The flow rate of the HPLC mobile phase was $0.7 \mathrm{~mL} / \mathrm{min}$. The needle electropolymerized for 60 minutes was used as the $\mu$ MISPE device. A chromatogram was obtained using the data acquisition system.

\subsection{Results and Discussion}

\subsubsection{In-situ electropolymerization}

To make a conventional MISPE device, a small amount of imprinted polymer (typically $50-200 \mathrm{mg}$ ) is packed into a column or a cartridge. Before packing, the bulk polymer must be crushed, ground, and sieved to provide particles of appropriate size. The grinding and sieving processes are rather time-consuming and the subsequent sedimentation process for the removal of fine particles results in a considerable loss of polymer material $[5,21]$. To solve this problem, our group has developed in-situ electropolymerization method for preparing MISPE devices. The molecularly imprinted nano-composite of PPy/CNTs (as MISPE sorbent) was formed inside a stainless steel syringe needle for use as a MISPE device. After the removal of the template, the device was used for extraction and pre-concentration of trace level OTA from red wine. The electrochemical method was employed since it is considered better for controlling the 
structure and morphology of electrodeposited PPy [38]. To form nano-composite of PPy/CNTs, the coating of polypyrrole film on the CNTs surface was considered as a crucial step $[43,45]$. PPy film can be formed from an electrolyte solution by electropolymerization at a potentiostatic or galvanostatic state. In this study, composite of PPy/CNTs was synthesized under potentiostatic conditions. The formed conducting polymer PPy served as a polymeric matrix for molecular imprinting while the CNTs provided the backbone of a three dimensional network of the composite material. Since the MISPE sorbent was grown directly inside the device, it did not need any subsequent packing in a time-consuming procedure. Thereafter, its applications would be easy and simple. Also the miniaturized device reduced the amount of sorbent required for packing the MISPE device, which is especially important in the case of MISPE if the template species are expensive.

\subsubsection{Optimization of the electropolymerization time}

To make a MIPPy/CNTs-modified syringe needle, it is very important to grow fairly rigid three-dimensional structure (or network) along the inner wall of the needle. Several methods reported in the literature regarding the preparation of $\mathrm{PPy} / \mathrm{CNT}$ composites, but most of these materials were in the form of PPy/CNT films [43, 45] or PPy/CNT nano-cables $[71,72]$. Teh et al. studied the film growth of $P P y / C N T$ composite by electropolymerization [73]. They described how the film started to grow by an island nucleation on the electrode surface and, with individual nuclei growing and enlarging, a continuous film would form at some point. The presence of CNTs provided more sites for nuclei formation such that there was more three-dimensional growth of PPy. Yu et al. discovered that CNTs could be tightly coated with a PPy layer and many neighboring CNTs were able to be joined together by the conductive polymer at a variety of angles [74]. In 
another study with synthesis of a CNT-PPy composite, nanotubes were also found joining to other nanotubes by means of the polymer [43]. Their studies demonstrated that there is a possibility that a three dimensional structure of CNT-PPy composite could be formed by carefully controlling the electropolymerization conditions or choosing a suitable pre-electropolymerization mixture.

In this work, a high concentration of CNTs ( $1.0 \%$ by weight) was used and the effect of electropolymerization time was studied. It was found that with the increase of the electropolymerization time, the higher backpressure of the MIPPy/CNTs-modified stainless steel needles exhibited. For the preparation of MIPPy/CNTs-modified stainless steel needles $(22 \mathrm{G})$, a reasonable backpressure was necessary to indicate that a three dimensional network existed inside the needle. However, when the electropolymerization time was prolonged up to 90 minutes, the modified needle was eventually clogged. It may be explained by the simple fact that more CNTs were joined together to block the needle. A suspension of nano-particles (20-30 $\mathrm{nm}$ in aggregate diameter) was used to test if the network was formed within the modified needles with electropolymerization time at 45 minutes and 60 minutes. After passing 3 drops of the suspension through the modified needle, the needle with a electropolymerization time for 60 minutes became clogged. By contrast, a blank needle and another needle electropolymerized for 45 minutes were able to pass the suspension in a continuous flow. This made us believe that a three-dimensional network of MIPPy-encapsulated carbon nanotubes was formed along the inner wall and extended inside the stainless steel needle. The electropolymerization time was optimized based on the backpressure of the MIPPy/CNTs-modified stainless steel needles (see the results in Table 3-1). 
Table 3-1 Electropolymerization time and backpressure test

\begin{tabular}{|c|c|c|}
\hline Electropolymerization Time(min) & Flow Rate Test Range(mL/min) & Backpressure \\
\hline 90 & $<0.1$ & high \\
\hline 60 & $0.1-1.2$ & reasonable \\
\hline 45 & $0.1-1.5$ & low \\
\hline
\end{tabular}

* The backpressure was considered too high, if the pump stopped automatically at a flow rate less than $0.1 \mathrm{~mL} / \mathrm{min}$.

* The backpressure was reasonable if a higher flow rate range (between $0.10 \mathrm{~mL} / \mathrm{min}$ and $1.2 \mathrm{~mL} / \mathrm{min}$ ) could be employed.

* There was no backpressure if the maximum flow rate of the pump $(1.5 \mathrm{~mL} / \mathrm{min})$ could be used and no shut down of the pump occurred.

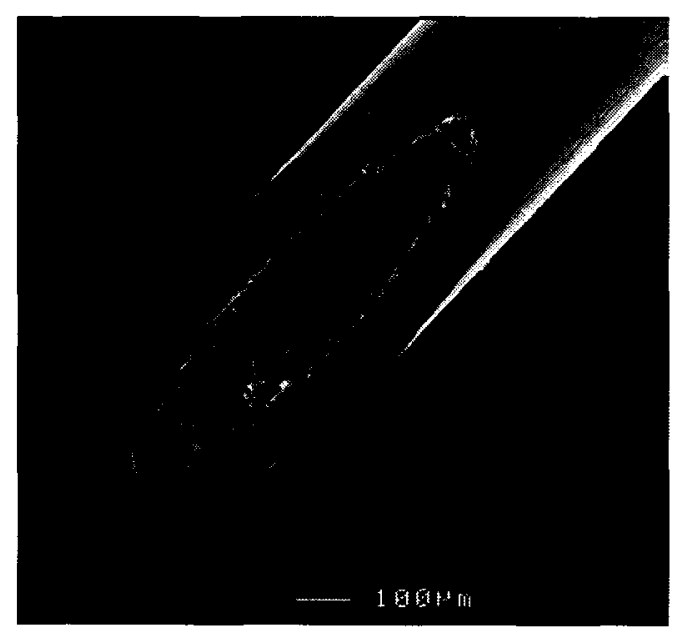

(a)

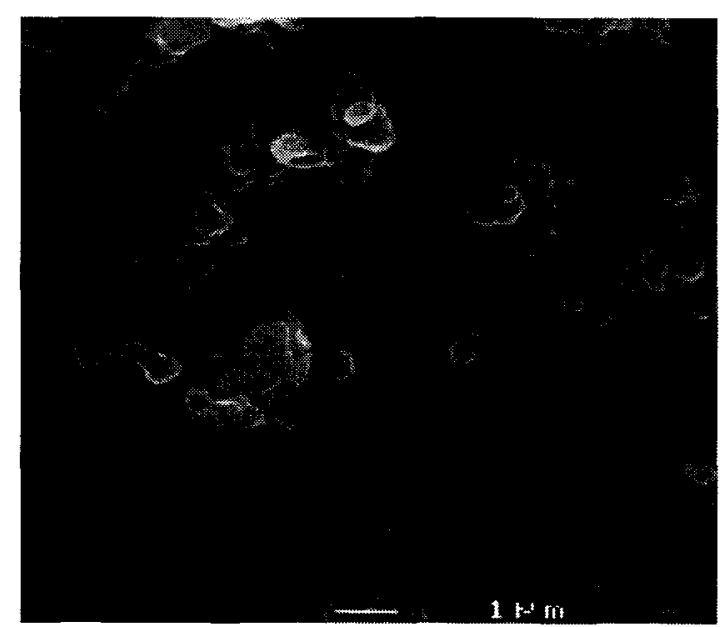

(b)

Fig. 3-1 SEM of MIPPy/CNTs on the inner wall of a modified stainless steel syringe needle. (a) Tip of the modified needle; (b) Circled area in (a) under higher magnification 


\subsubsection{Scanning electron microscopy image}

SEM was used to confirm the formation of the network inside the modified needle (at least at the tip), as illustrated in Fig. 3-1 (a) and (b). The circled area in Fig. 3-1 (a) shows that a small cluster of MIPPy/CNTs was formed at the tip of the inner wall of the syringe needle. The SEM image of this cluster in Fig. 3-1 (b) under higher magnification reveals that in the circled area of Fig. 3-1 (a) most of the CNTs are in the form of small bundles and most of the bundles have a diameter in the order of micrometers. Such small bundles with porous structure are believed to be very beneficial for the formation of the three dimensional network inside the modified needle and also are very beneficial for the $\mu$ MISPE performance, since most of the MIPPy-modified surfaces were readily accessible to OTA molecules for high-recovery binding (even at low OTA concentrations and fast sample flow rates). It can also be seen in Fig. 3-1 (b) that most of CNTs are coated with MIPPy and the encapsulated CNTs were joined to other encapsulated CNTs by means of MIPPy. The average diameter of PPy encapsulated CNTs is in the order of sub-micrometers and the length is about 1 micrometer.

\subsubsection{Choose of the eluting solvent for removal of temple OTA}

Removing the template OTA molecule from the polymeric matrix is a necessary step. It is not only to make the recognition site available for rebinding of the analyte, but also limit possible interference of any bleeding template with the analyte quantification. However, one problem with bulk MIPs is that some small amount of template remains strongly bound to the polymer despite the best attempts to extract it (as shown in Fig. 3-2 of G). This usually amounts to more than $1 \%$ of the amount of template given to the prepolymerization mixture and remains bound even after careful washing of the polymer. It 
may not constitute a problem in preparative separations or catalysis, but when the MIPs are used for sample preparation prior to analytical quantification of low levels of analytes, bleeding of this fraction will cause false positive results [24]. However, for imprinted nano-particles removal of template seems not to be a serious problem and some researchers even claimed that one superior characteristic of imprinted nano-particles is a better removal of the original template due to the large surface area available for template removal [31].

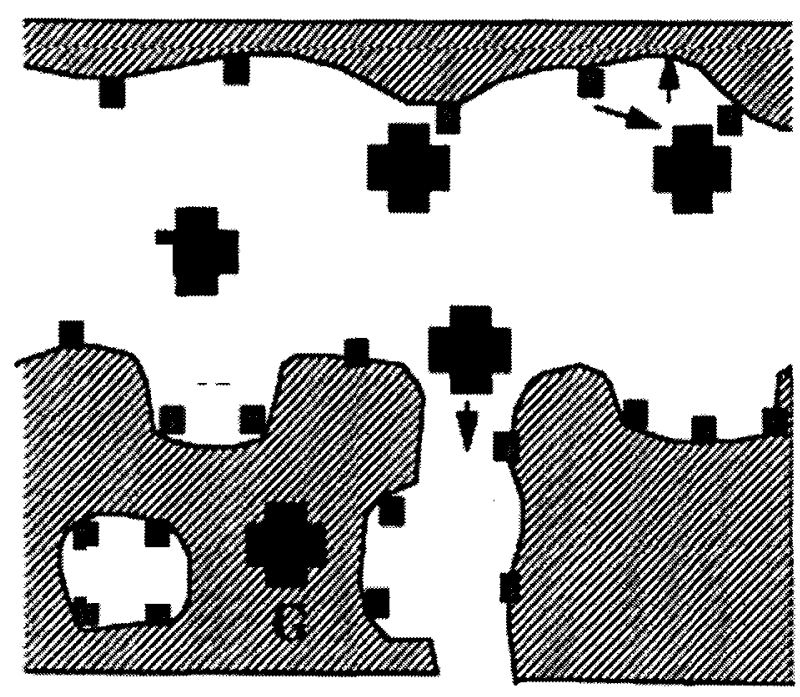

Fig. 3-2 Residue template in the polymeric structure of bulk MIPs G: residue template (modified from reference 24 )

In the case of MIPPy/CNTs, different eluting solvents were tested in order to select the optimal one for removing all template OTA. The choice of eluting solvent was based on the OTA solubility. As an organic acid, OTA is soluble in common polar organic solvents as acetonitrile and methanol [75]. It can also dissolve in water under neutral and alkaline conditions [75]. Based on this property, organic solvents (such as methanol and acetonitrile) and two alkaline aqueous solutions were evaluated/tested for use as eluting solvents. To 
prepare aqueous eluting solutions, a strong organic base TEA was used. Previous studies in our group discovered that three different ion-pairs could be formed: $\left[\mathrm{NH}_{4}^{+}-\mathrm{OTA}^{2-}-\mathrm{TEA}^{+}\right]$, $\left[\mathrm{NH}_{4}^{+}-\mathrm{OTA}^{2-}-\mathrm{NH}_{4}^{+}\right]$and $\left[\mathrm{TEA}^{+}-\mathrm{OTA}^{2-}-\mathrm{TEA}^{+}\right]$. These three ion-pairs may contribute to the better removal of template OTA from the polymeric matrix [76]. Thereafter, a solution of $1 \%$ and $2 \%$ TEA (by volume) in HPLC mobile phase were chosen. The needles with a electropolymerization time of 60 minute were washed with different eluting solutions. After no OTA was detected in the eluates, the needles were stored overnight for bleeding test. The bleeding test was carried out by using $50 \mu \mathrm{L}$ eluting solvent to wash the needle again next day, and the eluate was collected for analysis by HPLC-FD. The results of the washing experiment were summarized in Table $3-2$. It shows that solutions of $1 \%$ and $2 \%$ TEA in HPLC mobile phase are more effective to remove template OTA and no significant template bleeding was observed for needles washed with these two solutions. Although there is no significant difference between $1 \%$ and $2 \%$ TEA in HPLC mobile phase to serve as a good eluting solvent, $2 \%$ TEA was usually used to remove template OTA from the MIPPy/CNTs. It is also noteworthy that a relatively small volume was required to remove temple OTA from MIPPy/CNTs-based devices as compared to another study with bulk OTA selective-MIPs prepared by Jodlbauer et al.[58]. In their work, to remove template OTA, continual washing was conducted overnight at a flow rate of $1 \mathrm{~mL} / \mathrm{min}$. We attribute the easy removal of the template to the nanostructure of MIPPy/CNTs that provides a large surface area accessible to the eluting solvent. Moreover, the MIPPy film/coating was very thin (which is easy for the infiltrating solvent to diffuse through).

Even no template bleeding was observed, one or two quick washings with 50-100 $\mu \mathrm{L}$ of $1 \%$ TEA (prepared in HPLC mobile phase) was still employed before every reuse of the 
modified needle to prevent any false positive (without repeating the HPLC analysis of eluate).

Table 3-2 Choose of eluting solvent for removal of template OTA

\begin{tabular}{|c|c|c|c|}
\hline Eluting Solvent & $\begin{array}{c}\text { Volume used to } \\
\text { wash the needle } \\
(\mathrm{mL})\end{array}$ & $\begin{array}{c}\text { Template washed } \\
\text { off the needle } \\
(\mathrm{ng})\end{array}$ & $\begin{array}{c}\text { Bleeding after storing } \\
\text { the needle overnight }\end{array}$ \\
\hline Methanol & 30 & 27.5 & Yes \\
\hline Acetonitrile & 35 & 26.0 & Yes detectable at LOD \\
\hline $1 \%$ TEA in mobile phase & 30 & 28.7 & Not detectable at LOD \\
\hline $2 \%$ TEA in mobile phase & 30 & 28.9 & \\
\hline
\end{tabular}

\subsubsection{Effect of sample pH on MIPPy/CNT- $\mu$ SPE-PE-HPLC-FD}

The success of molecular imprinting depends not only on the resulting polymer structure but also on the sample medium [77]. The condition of the sample should suppress the non-specific interactions without disrupting the selective interactions between the MIP and the target molecule. In a study with recognition properties of MIP specific for OTA, it was found that variations in buffer concentration, $\mathrm{pH}$ and organic solvent had a profound effect on the MIP recognition properties [78]. Yu et al. discovered in the study with PPy-modified stainless steel frits for $\mu$ SPE of OTA that OTA in anionic form was expected to be more efficiently incorporated than the neutral form, based on the anion versus cation exchange properties of PPy. However, a higher recovery was found at a low pH of 2.5 [40].

In the present work, to better understand the binding mechanism of analyte OTA toward MIP, $\mathrm{pH}$ effect of the sample on the extraction efficiency was investigated. Standard 
OTA solutions $(1.0 \mathrm{ng} / \mathrm{mL})$ were prepared with different buffers $(\mathrm{pH}=1.5,2.5$ and 4.0$)$ and the effect of sample $\mathrm{pH}$ was studied. The rebinding efficiency was characterized by the MIPPy/CNT- $\mu$ SPE-PE-HPLC-FD peak area. A dependence of the extraction performance (in terms of the HPLC peak area) on the $\mathrm{pH}$ value of sample solution was observed (as shown in Fig. 3-3). The largest peak area (or recovery of OTA) was obtained at pH 2.5. At a lower $\mathrm{pH}$ of 1.5 , the HPLC-FD peak area was significantly decreased. With the increase of the sample $\mathrm{pH}$ values $(\mathrm{pH}=4.0)$, the peak area was even more significantly decreased. The OTA molecule, as a weak organic acid with $\mathrm{pK}_{\mathrm{a}}$ values of about $\sim 3.5$ (carboxyl group of phenylalanine) and $\sim 7.5$ (phenolic group), was present in the fully protonated (neutral) form in a medium with $\mathrm{pH}=3.0$ [79]. The neutral form of analyte OTA molecule can interact with the hydrogen atom in the $\mathrm{N}-\mathrm{H}$ group of the PPy unit which served as a hydrogen bond donor [80]. At pH 2.5, a complex of the analyte OTA molecule and the EDGMA-crosslinked PPy may have been formed through hydrogen bonding and hydrophobic interactions [40]. Kanekiyo et al also believed that adjusting the buffer $\mathrm{pH}$ could control the binding interaction between analyte and MIPs since the $\mathrm{pH}$ of the buffer greatly influence the formation of labile complexes between the analyte and the polymer [81]. In this study, with the increase of the sample $\mathrm{pH}$ values $(\mathrm{pH}=4.0)$, the MIPPy/CNT- $\mu$ SPE-PE-HPLC-FD peak area was significantly decreased. It may be as a consequence of the ionization of the carboxyl group at higher $\mathrm{pH}$, which disturbed the hydrogen bonding between OTA and PPy. At a lower $\mathrm{pH}$ of 1.5, the HPLC-FD peak area was significantly decreased. This may be caused by OTA degradation via acid catalyzed hydrolysis to phenylalanine and ochratoxin $\alpha$ [55]. However, hydrolysis of the amide requires forcing conditions and it is unlikely to be significant at room temperature even at 
$\mathrm{pH}$ 1.5. Further investigation would be required to gain a better understanding of this low pH effect.

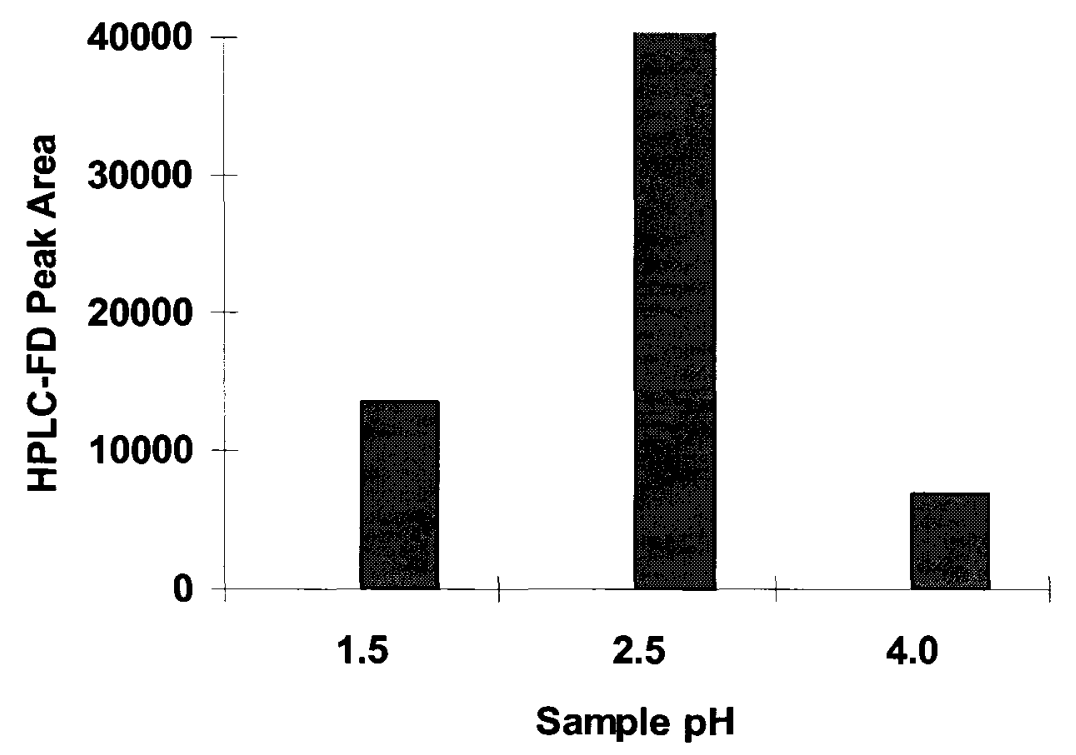

Fig. 3-3 Effect of sample solution pH on MIPPy/CNT- $\mu$ SPE-PE-HPLC-FD

\subsubsection{Effect of sample uptaking flow rate on MIPPy/CNT- $\mu$ SPE-PE -HPLC-FD}

The flow rates were studied over a syringe pumping range from 0.05 to $0.50 \mathrm{~mL} / \mathrm{min}$, using $0.5 \mathrm{~mL}$ of spiked red wine (containing $0.5 \mathrm{ng} / \mathrm{mL}$ OTA) as the working samples. The test was carried out in the presence of the red wine matrix since the structural analogues existing in the red wine may compete with OTA for the binding sites. We attempted to understand the binding activity of OTA towards MIP recognition sites in the presence of interferences. Taking advantage of this experiment, the optimal sample uptaking flow rate for red wine analysis was also obtained experimentally. As shown in Fig. 3-4, it is clear that OTA was enriched by MIPPy/CNTs- $\mu$ SPE at flow rates up to $0.10 \mathrm{~mL} / \mathrm{min}$ and a decrease of HPLC-FD peak areas at $0.09 \mathrm{~mL} / \mathrm{min}$ or higher rate was observed. At a flow rate of 0.10 $\mathrm{mL} / \mathrm{min}$, it only took 5 minutes to pre-concentrate (extract) $0.5 \mathrm{~mL}$ of red wine sample 
spiked at the $0.5 \mathrm{ng} / \mathrm{mL}$ OTA level. In another study with over-oxidized PPy colloids imprinted with L-lactate, it was found that a much larger surface area plus a short diffusion distance of nano colloid particles allowed the system to reach absorption equilibrium in $<10$ $\min [67]$

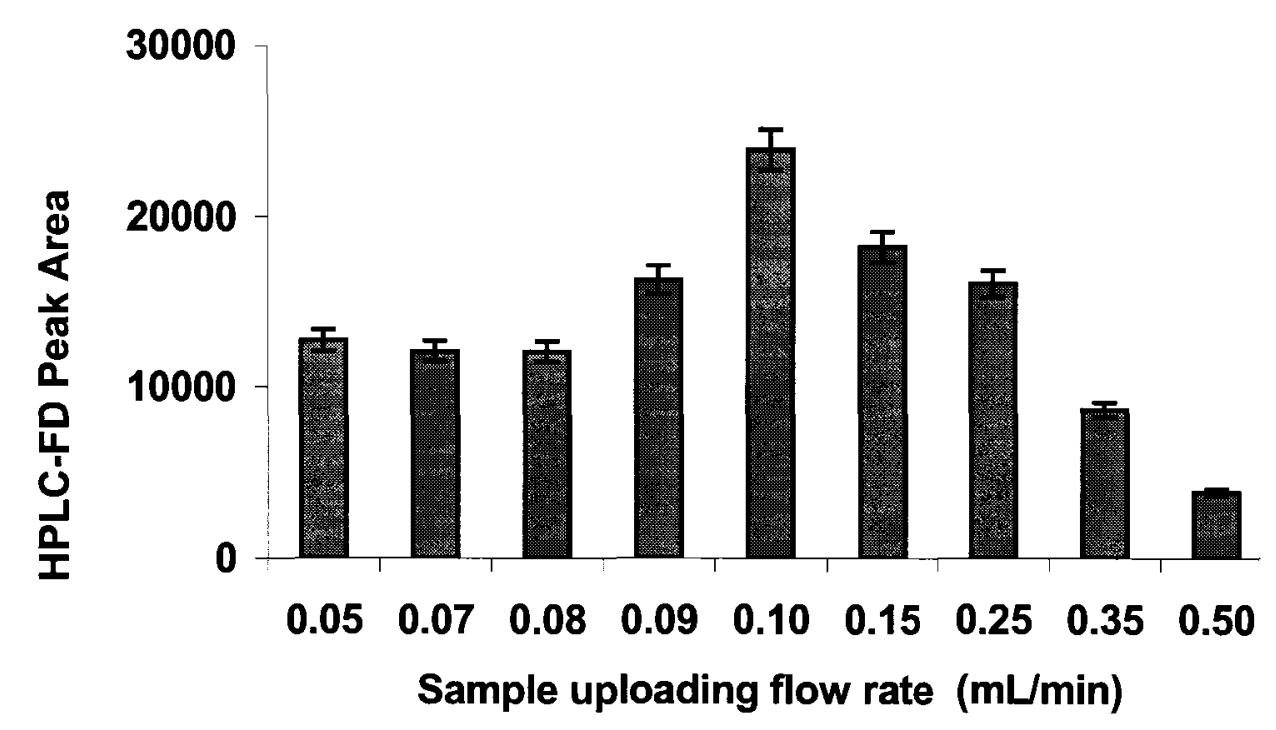

Fig. 3-4 Effect of pre-concentration flow rate on MIPPy/CNT- $\mu$ SPE-PE-HPLC-FD

In our work, addition of CNTs to MIPSPE is believed to contribute to enlargement of the surface area (see section 4.3.7). We assumed that the larger surface area of MIPPy/CNTs sorbent would improve the accessibility of the binding sites, which enabled faster diffusion and mass transfer of analyte OTA to the binding cavity. Hence the binding process of the analyte could be completed in a shorter period of time. For trace level analysis, a rapid sample pretreatment step is very important and sometimes it may hold the key for success in an analytical method development. Since the extraction procedure developed in this thesis significantly shortened the processing time, it is very attractive for a fast analysis. 


\subsubsection{Standard calibration curve}

A typical calibration plot (MIPPy/CNT- $\mu$ SPE-PE-HPLC-FD peak area versus OTA concentration) was generated by spiking $0.5 \mathrm{~mL}$ of blank red wine up to OTA concentrations of $0.1,0.3,0.5,0.7$, and $0.8 \mathrm{ppb}$ (or $\mathrm{ng} / \mathrm{mL}$ ) as shown in Fig. 3-5. The standard calibration curve was constructed to show that the MIPPy/CNTs- $\mu$ SPE-HPLC-FD peak area was linearly related $\left(\mathrm{R}^{2}=0.9937\right)$ to OTA concentration (or mass of OTA loaded on the MIPPy/CNTs-modified syringe needle) in the working range from 0.10 to 0.80 $\mathrm{ng} / \mathrm{mL}$. The limit of detection $\left(\mathrm{LOD}=3 \sigma_{\mathrm{B}} / \mathrm{b}, \mathrm{n}=7\right.$, R.S.D. $=6 \%$ ) and limit of quantitation $\left(\mathrm{LOQ}=10 \sigma_{\mathrm{B}} / \mathrm{b}, \mathrm{n}=7\right)$ were found to be $0.04 \mathrm{ppb}$ (or $\mathrm{ng} / \mathrm{mL}$ ) and $0.12 \mathrm{ppb}$ (or $\mathrm{ng} / \mathrm{mL}$ ) based on the standard deviation of the blank $\left(\sigma_{\mathrm{B}},\right)$ and the slope of the calibration curve $(\mathrm{b})$, and the relative standard deviation (R.S.D.) was better than $6 \%$. The MIPPy/CNTs- $\mu$ SPE-HPLC-FD method, developed and tested in this study, compares favourably with a LOD of $0.1 \mathrm{ng} / \mathrm{mL}$ OTA for direct HPLC analysis by using an alkaline mobile phase [50] and a LOD of $5 \mathrm{ng} / \mathrm{mL}$ for $\mathrm{C}_{18}$ solid phase extraction coupled with HPLC analysis [82]. 


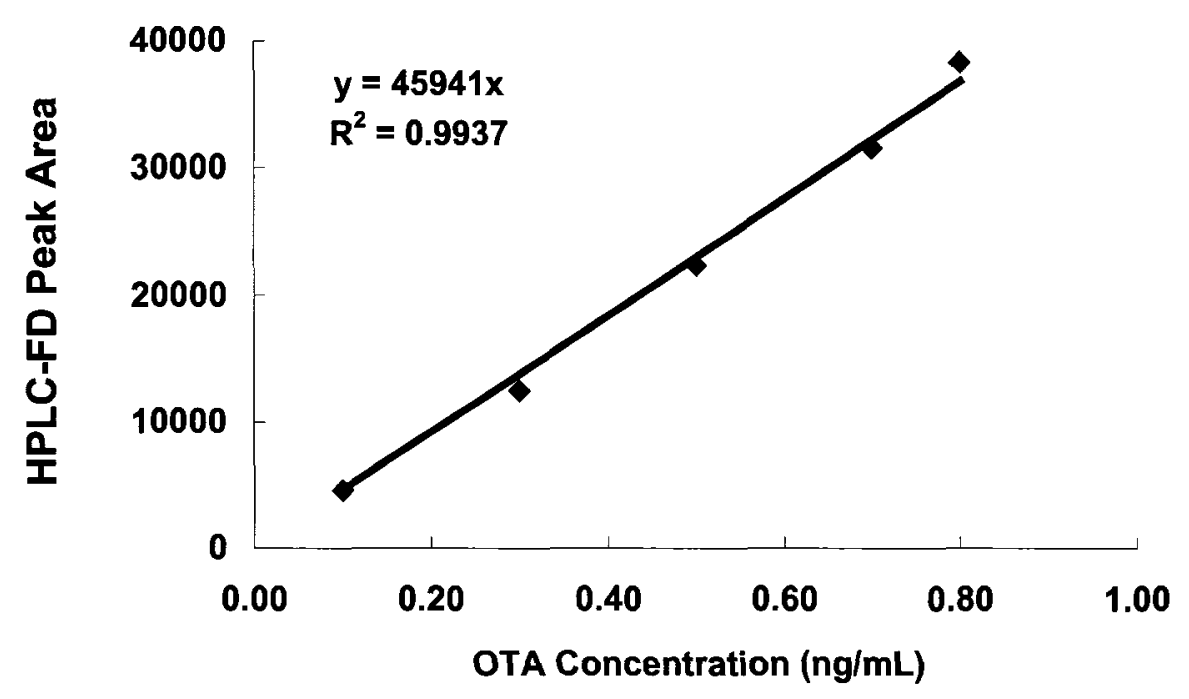

Fig. 3-5 Standard calibration curve of MIPPy/CNTs-SPE-PE-HPLC-FD analysis with spiked red wine samples

\subsubsection{Application of MIPPy/CNTs- $\mu$ SPE-PE-HPLC-FD to red wine analysis}

We employed this MIPPy/CNTs- $\mu$ SPE-HPLC-FD method to analyze Italian red wines that were purchased from local stores. No OTA was detectable in the Italian red wine. Fig. 3-6 shows a typical chromatogram (HPLC mobile phase flow rate $=0.7 \mathrm{~mL} / \mathrm{min}$ ) from the MIPPy/CNTs- $\mu$ SPE-HPLC-FD analysis of $0.5 \mathrm{ng} / \mathrm{mL}$-OTA-spiked red wine samples $(0.5$ $\mathrm{mL}$ ). The OTA peak appeared at a retention time of $9.28 \mathrm{~min}$, which was well separated from the earlier peaks of wine matrix components. The ultimate merit of this MIPPy/CNTs- $\mu$ SPE technique is that only a small volume $(0.5 \mathrm{~mL})$ of red wine sample is required and the extraction time is as short as $5 \mathrm{~min}$. When a conventional octadecyl silane $\left(\mathrm{C}_{18}\right)$ solid phase column was used previously, a much larger volume $(100 \mathrm{~mL})$ of sample was required [56]. The smaller sample volume requirement and fast binding of the analyte to recognition sites in the extraction step enabled the development of a rapid analytical method for screening of OTA contamination in red wines. Compared to another OTA selective-MIP based SPE method, in which a larger sample volume $(10 \mathrm{~mL})$ was required 
and the extraction time was 50 minutes [59], the method developed in this thesis is much preferred for practical applications.

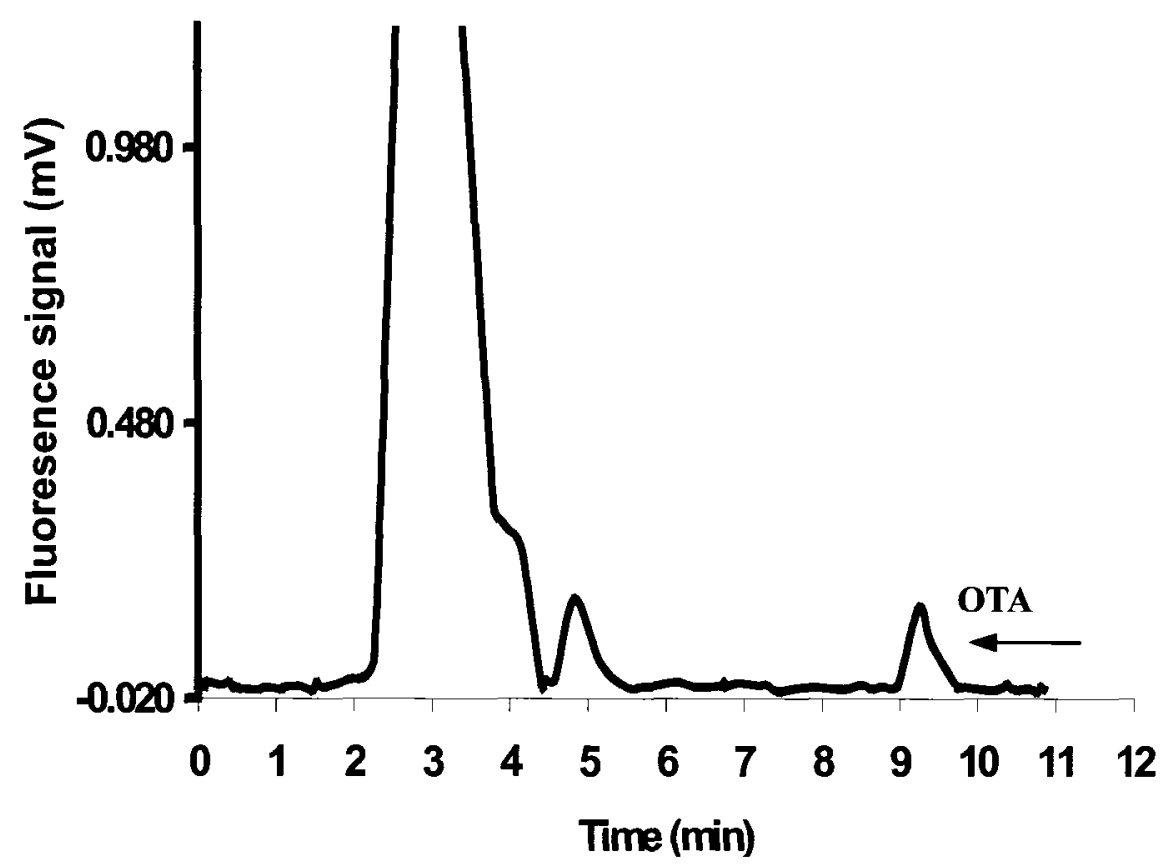

Fig. 3-6 Analysis of 0.5 ppb-OTA-spiked red wines by MIPPy/CNTs- $\mu$ SPE-PE -HPLC-FD after enrichment of OTA from $0.5 \mathrm{~mL}$ of red wine (Flow rate of HPLC mobile phase: $0.7 \mathrm{~mL} / \mathrm{min})$.

\subsubsection{Reusability of MIPPy/CNTs-modified syringe needles}

The MIPPy/CNTs-modified stainless steel needle seemed to be stable and robust under ordinary lab operation conditions. Our observation showed that its extraction efficiency remained relatively constant over 3 months (used more than 60 times). We attributed this long-term reusability to the stability of the classical technology of cross-linking PPy with EGDMA, thereby encapsulating the CNTs permanently. 


\subsection{Conclusion}

A new $\mu$ MISPE device has been developed using molecularly imprinted polypyrrole encapsulated carbon nanotubes as the sorbent. The MIPPy/CNTs composite was grown using electrochemical polymerization, in which the conductive polymer and nanotubes were simultaneously deposited along the inner wall of the stainless steel syringe needle to form a three-dimensional network. The resulting needle exhibited a significant binding capacity for OTA and effectiveness to remove the interfering components of complex red wine matrices. The MIPPy/CNTs-modified needle as a $\mu$ MISPE device afforded an enriched sample that is suitable for sensitive HPLC-fluorescence determination of OTA. Compared to the popular IA columns for OTA analysis, the MIPPy/CNTs-modified needle is much more robust and cost-effective for repeated use. When compared to conventional $\mathrm{C}_{18}$ SPE columns, the MIPPy/CNTs-modified needle offers a lower detection limit $(0.04$ $\mathrm{ppb})$ and uses a smaller volume of wine sample $(0.5 \mathrm{~mL})$. This new preparation technique can be applied to needles in various lengths and shapes. Therefore it can readily handle small sample volumes (several $\mu \mathrm{L}$ ) as well as large volumes (several $\mathrm{mL}$ ), even for autosamplers that are coupled online to HPLC and liquid chromatography-mass spectrometry (LC-MS).

The present technique demonstrates that MIP combined with nano-technology can lead to the development of novel extraction procedures and new sample preparation devices. In the next chapter, a new nano-material (quantum dots) was employed to build three-dimensional structures inside a stainless steel needle. We believe that those nano-scale structures can further increase the total surface area of MIPPy film, thus dramatically enhancing the extraction efficiency (or recovery). 


\section{CHAPTER 4}

Encapsulation of Quantum Dots and Carbon Nanotubes

with Polypyrrole in a Syringe Needle for Automated Molecularly

Imprinted Solid Phase Extraction of Ochratoxin A in Red Wine Analysis 


\subsection{Introduction}

Nano-particles (NPs) have opened new avenues of sensing and instrumentation research. Quantum dots (QDs) are special semiconductor nano-crystals whose electrons occupy well defined discrete quantum states. When QDs are photo-excited, electron-hole pairs are generated and upon their recombination fluorescence light is emitted [83]. Due to their unique photophysical properties, tremendous interest is developing in the use of QDs as fluorescent labels and probes for biochemical applications [84]. QDs can be modified at the surface with a conjugate, which interacts with an external agent by physical (i.e. recognition) or chemical means [85]. They appeal to bioengineering because specific biomolecules on their surface determine the binding affinity [86], as afforded by the bioconjugation of a highly specific monoclonal antibody [87]. These glowing nano-crystals can be ferried to specific molecules on the cell surface [88] to achieve labeling of the transfected cells [89]. The similar dimensions of biomolecules (such as enzymes, antibodies or DNA) and metallic or semiconductor NPs enable the synthesis of biomolecule-NP hybrid systems where the unique electronic, photonic and catalytic properties of NPs are combined with the specific recognition and biocatalytic properties of biomolecules $[90,91]$. The biomolecule-NP hybrid systems are becoming the material of choice for a variety of analytical applications $[92,93] . \quad$ Novel research may arise from their bulk, surface and colloidal properties. As the nano-crystals form during manufacture, their shape changes and every crystal begins to expose more edges and corners. In other words, QDs have more surface area on which to perform chemical reactions. These nano-crystals can potentially advance analytical applications that depend on surface chemistry [94]. In particular, QDs may increase 
the sensitivity of solid phase preconcentration (SPP) because of their enormous surface area-to-volume ratio [95] and specific surface area [96, 97]. Although QDs differ in sizes and surface structures, they have similar specific surface area (typically 120-150 $\mathrm{m}^{2} / \mathrm{g}$ ) for chemical interactions $[98,99]$. Moreover, low non-specific binding has been shown at intermediate sizes [100]. In previous studies, QDs were immobilized on a polylysine-coated glass surface [101]. A thin-film of QDs-polymer mix was spin-coated onto a silicon substrate, thereby creating a hybrid device [102]. Alternatively, multi-walled carbon nanotubes (CNTs) are of interest for composite materials due to their good electronic properties and high mechanical stability [103]. They are commercially available with the following specifications: external diameter $=$ $10-30 \mathrm{~nm}$, length $=0.5-50 \mu \mathrm{m}$, purity $>95 \%$, specific surface area $=4-100 \mathrm{~m}^{2} / \mathrm{g}$. The utilization of CNTs as building blocks for nano-devices can readily be realized. Different nanoparticles can be coupled to functionalized carbon nanotubes (CNTs) in a uniform and controllable manner [104], and hetero-junctions of QDs and CNTs could become better alternatives for the synthesis of nanoscale devices. Ravindran et al presented a technique to integrate QD at the ends of CNTs and reported a novel CNT-QD-CNT heterostructure with detailed chemical and physical characterization of the heterojunctions [105]. Li et al demonstrated that QDs could be selectively assembled on the aligned CNT films with desired patterns [106]. Molecular imprinting is tantamount to creating a memory cavity which is capable of selectively and reversibly rebinding the template. Polypyrrole (PPy) is excellent for electrochemical preparation of molecularly imprinted polymers (MIPs) due to its stability under mild preparation conditions of room temperature and $+0.85 \mathrm{~V}$ versus $\mathrm{Ag} / \mathrm{AgCl}$ (even when a cross-linker is 
added). Conducting PPy is a biocompatible polymer matrix wherein biochemical molecules can be incorporated by way of doping [107, 108]. Functionalization with a wide range of bioorganic templates (such as ochratoxin A) is possible. Desirable properties such as a large surface area was achieved by electrodeposition of PPy on macroporous stainless steel frits [61], and molecularly imprinted polypyrrole (MIPPy) has been used to encapsulate CNTs inside the frit pores for selective micro solid phase preconcentration ( $\mu$ SPP) [30]. More interestingly, a syringe needle can be packed with MIPPy-encapsulated CNTs for $\mu$ MISPE [70].

With the introduction of new advanced materials that provide applications in the development of sensing and instrumentation research, new sample preparation techniques are in great need. Despite recent advances in analytical methodologies, many of the conventional sample preparation methods (which are usually done off-line or manually) are retained. They are often the slowest and the most costly part of the analytical process, especially if multi-step procedures are used. It is not surprising that researchers have invested considerable effort in the development of automated sample preparation techniques during the last few years [109]. Obviously, the automation and integration of sample preparation and analytical method into one automated step are very desirable [110]. Unfortunately, many of the conventional procedures are not amenable to automation [109]. Solid phase extraction as one of the widely used sample pretreatment techniques) allows straightforward automation, and it has been combined with molecularly imprinting in order to improve selectivity [111]. In the common approach of automated MISPE, the MIP is packed in a stainless steel cartridge to be used in a column switching approach prior to HPLC analysis [112]. 
The present chapter reports an automation of integrated sampling (extraction)/pulsed elution/injection (detection) method for determination of trace-level analyte (OTA) in a complex matrix (red wine). It is focused upon the establishment of a rapid, facile and fully automated MISPE procedure which is made/proven feasible by developing a new way to immobilize various nano-composites as sorbents in the autosampler needle [70]. The use of nano-composite based sorbent opens up possibilities for miniaturization of the SPE device (going down in size scale from a cartridge or micro-column to a syringe needle). The MIPPy/CNTs/QDs- modified syringe needle can be used as a HPLC autosampler injection needle.

Knowing that conductive PPy is technically a semiconductor, MIPPy can be effectively used as macromolecular glue to immobilize semiconductor QDs on the CNTs surface during electrodeposition. The expected benefits would be a significantly larger total surface area and shorter diffusion distances for more effective $\mu$ MISPE of ultratrace ochratoxin A (OTA) in red wine. OTA is a mycotoxin produced by specific types of fungi in wine products; it is well known as a potent nephrotoxin, teratogen and carcinogen to humans [113]. The MIPPy/CNTs/QDs-modified needle can be mounted in an autosampler for sequential HPLC-FD analysis of red wine samples. Operator-free processing of red wine samples $\geq 1.0 \mathrm{~mL}$ containing OTA at $<0.02 \mathrm{ppb}$ concentration levels is feasible. Different pulsed elution (DPE) strategies are programmable on the HPLC autosampler to verify that MIPPy is truly a MIP with specific recognition for OTA if all red wine matrix components can be differentially washed out. 


\subsection{Experimental}

\subsubsection{Preparation of MIPPy/CNTs/QDs-modified stainless steel syringe needle}

Three different OTA concentrations (100, 150 and $300 \mu \mathrm{M}$ OTA) were added when Py was electropolymerized within each 22-gauge, 1.5-inches-long (for a perfect fit to the autosampler) stainless steel syringe needle (BD, Franklin Lakes, NJ, USA). For electrochemical preparation of MIPPy-encapsulated CNTs/QDs, the needle was first filled with the pre-polymerization mixture (100-300 $\mu \mathrm{M}$ OTA, 0.1 M Py, 0.1 M TBAP, 0.2 M EGDMA, $1 \%$ by weight CNTs and acetonitrile as the solvent) and then connected to serve as the working electrode. The set-up of the electropolymerization was described in section 2.4. The applied potential was switched off after 30 minutes of electropolymerization. Then, the needle was filled with another pre-polymerization mixture (100-300 $\mu$ M OTA, 0.1 M Py, 0.1 M TBAP, 0.2 M EGDMA, 1\% by weight QDs, and acetonitrile as the solvent ) for more electropolymerization over $15 \mathrm{~min}$.

After the electropolymerization, the template OTA was removed as described in section 2.7. The effect of template OTA concentration on recovery was characterized by automated $\mu$ MISPE-HPLC-PE-FD analysis (described in section 2.9) with $1.0 \mathrm{~mL}$ OTA standard solution $(1.0 \mathrm{ppb})$ as working solution. A plot of recovery vs the template OTA concentration in the pre-electropolymerization mixture was obtained.

\subsubsection{Measurement of total surface area by pulsed amperometry}

The working electrode consisted of a MIPPy/CNTs/QDs-modified needle. A platinum wire was used as the counter electrode, and an $\mathrm{Ag} / \mathrm{AgCl}$ electrode was used as the reference electrode. A special cell was constructed in the laboratory for the measurement of total surface area by pulsed amperometry, with the three electrodes 
connected to a PalmSens electrochemical analyzer (Palm Instruments, Houten, Netherlands) as illustrated in Fig. 4-1. For pulsed amperometric detection (PAD) analysis, the working electrode potential was pulsed between $-0.2 \mathrm{~V}$ and $+0.4 \mathrm{~V}$ over 0.2 seconds (as described by Gooding [114]) to alternate the polymer from a neutral to oxidized state when a $\mathrm{Na}_{2} \mathrm{SO}_{4}$ standard solution was running through the modified needle at a flow rate of $50 \mathrm{~mL} / \mathrm{hr}$ from a syringe pump. The PAD current was recorded every 1.0 second on a pocket PC (Hewlett-Packard Compaq iPAQ 3950, London, UK). A summary of the parameters used in PAD analysis is presented in Table 4-1. The pocket PC had a graphical user interface (PalmTime) that automatically plotted current versus time. This allowed the user to wait for the current (which indicated the total surface area) to stabilize until no fluctuations were observed. The pocket PC also stored the data in a file that could be transferred to a desktop computer for import to an Excel spread sheet.

Table 4-1 Parameters used in the PalmTime program for PAD analysis (version 1.35)

\begin{tabular}{|l|l|l|}
\hline Parameter & Setting & Description \\
\hline E & $-0.20 \mathrm{~V}$ & The constant potential applied. \\
\hline E pulse & $+0.40 \mathrm{~V}$ & The pulsed potential. \\
\hline T pulse & 0.2 seconds & The duration of each potential pulse. \\
\hline T interval & 1 second & The frequency at which the current was recorded. \\
\hline T run & $100-400$ seconds & This varied depending on desired duration of each run. \\
\hline
\end{tabular}




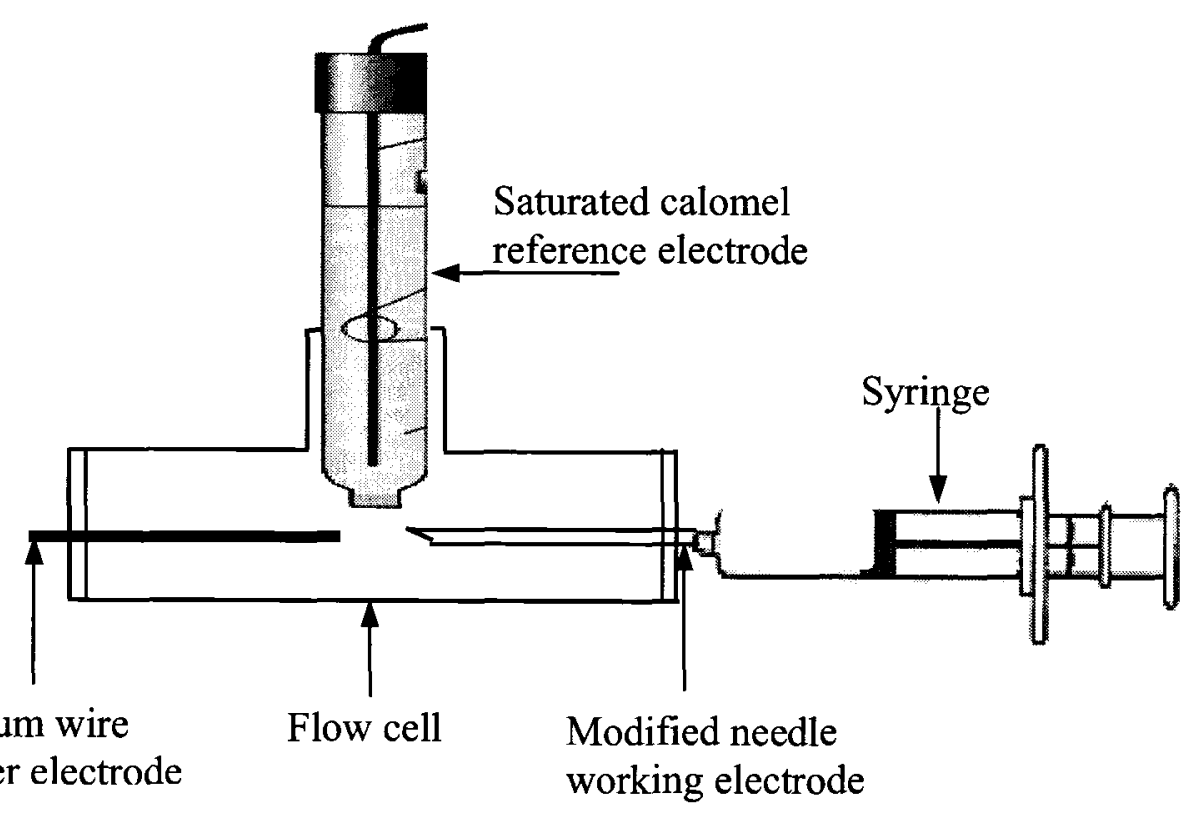

Fig. 4-1 Schematic diagram of special cell constructed for measurement of total surface area by pulsed amperometry, with the three electrodes connected to PalmSens electrochemical analyzer.

\subsubsection{Effect of analyte OTA concentration on \%recovery of MIPPy/CNTs/QDs- $\mu$ SPE -PE-HPLC-FD}

A sample of $1.0 \mathrm{~mL}(0.02-3.0 \mathrm{ppb}$ or $\mathrm{ng} / \mathrm{mL})$ OTA standard solution was analyzed according to the procedure described in 2.9. All samples were acidified with $1.0 \mathrm{M} \mathrm{HCl}$ ( $1 \%$ by volume, $\mathrm{pH}=2.5$ ). The MIPPy/CNTs/QDs-modified needle was prepared by using $300 \mu \mathrm{M}$ OTA present in the pre-electropolymerization mixture. The recovery was calculated (for calculation of recovery see section 2.10) and plotted against the concentration of OTA standard solution.

\subsubsection{Standard calibration curve for MIPPy/CNTs/QDs- $\mu$ SPE-PE-HPLC-FD analysis of OTA}


A plot of MIPPy/CNTs/QDs- $\mu$ SPE-PE-HPLC-FD peak area vs. the concentration of OTA standard solution was obtained over the OTA range from $0.02-3.0 \mathrm{ppb}$ (or $\mathrm{ng} / \mathrm{mL}$ ). All samples were acidified with $1.0 \mathrm{M} \mathrm{HCl}(1 \%$ by volume, $\mathrm{pH}=2.5)$. The MIPPy/CNTs/QDs-modified needle was prepared by using $300 \mu \mathrm{M}$ OTA present in the pre-electropolymerization mixture. The automated $\mu$ MISPE-HPLC-PE-FD analysis was conducted as described in section 2.9. The limit of detection $\left(\mathrm{LOD}=3 \sigma_{\mathrm{B}} / \mathrm{b}\right)$ and limit of quantitation $\left(\mathrm{LOQ}=10 \sigma_{\mathrm{B}} / \mathrm{b}\right)$ were determined based on standard deviations of the blank $\left(\sigma_{B}\right)$ and the slope of the calibration curve (b).

\subsubsection{Automated direct HPLC-FD analysis of red wine for OTA}

All red wine samples were filtrated with $0.45-\mu \mathrm{m}$ pore size filter (Nylon ID $13 \mathrm{~mm}$, ExpediGlass) and acidified with $1.0 \mathrm{M} \mathrm{HCl}$ ( $1 \%$ by volume). For direct HPLC-FD analysis of red wine samples, a blank needle was used and $200 \mu \mathrm{L}$ of red wine or $0.5-\mathrm{ppb}$ $(\mathrm{ng} / \mathrm{mL})$ OTA spiked red wine was automatically uptaken onto the autosampler injection valve (see section 2.8) and injected onto the HPLC-FD for chromatographic analysis. The conditions of HPLC-FD analysis were described in section 2.3 with a HPLC mobile phase flow rate of $0.7 \mathrm{~mL} / \mathrm{min}$. The chromatograms were recorded by the Peak Simple data acquisition system.

\subsubsection{Automated MIPPy/CNTs/QDs- $\mu$ SPE-PE-HPLC-FD analysis of red wine for OTA}

The sample preparation was the same as described in section 4.2.5. The MIPPy/CNTs/QDs modified needle was replaced the blank needle. A $1.0 \mathrm{~mL}$ of red wine or $0.5-\mathrm{ppb}(\mathrm{ng} / \mathrm{mL})$ OTA spiked red wine was extracted, pulse eluted (PE) and analyzed according to the procedure described in section 2.9 . The chromatogram was 
recorded by the data acquisition system and the recovery was calculated (for calculation of recovery see section 2.10 ).

\subsubsection{Choose of eluting solvent for differential pulsed elution}

DDW/85\% $\mathrm{H}_{3} \mathrm{PO}_{4}(60: 1$ by volume) and $1.0 \mathrm{M} \mathrm{HCl}$ were studied as differential pulsed elution (DPE) solvent for the investigation of the performance of DPE with MIPPy/CNTs/QDs-modified needle. The Italian red wine was used as the working sample. The sample preparation was the same as described in section 4.2.5. The DPE was performed according to the procedure established in section 2.9 .

The washing efficiency of each DPE or FPE was calculated by comparing the peak area of matrix peak or OTA peak obtained to the total peak area of matrix peak and OTA peak obtained in three DPEs and one FPE.

\subsubsection{Automated MIPPy/CNTs/QDs- $\mu$ SPE-DPE-HPLC-FD analysis of red wine for OTA}

An Italian and Canadian red wine sample were filtrated with $0.45-\mu \mathrm{m}$ pore size filter (Nylon ID $13 \mathrm{~mm}$, ExpediGlass) and acidified with $1.0 \mathrm{M} \mathrm{HCl}$ (1\% by volume). A 1.0 $\mathrm{mL}$ of red wine or $0.5-\mathrm{ppb}(\mathrm{ng} / \mathrm{mL})$ OTA spiked red wine was extracted, differential pulse eluted (DPE) and detected according to the procedure described in section 2.9. The chromatogram was recorded by the data acquisition system and the recovery was calculated (for calculation of recovery see section 2.10 ).

\subsection{Results and Discussion}

\subsubsection{Autosampler system for MIPPy/CNTs/QDs- $\mu$ SPE-PE-HPLC-FD analysis}

In this study, we developed a method where sampling, extraction/clean-up and injection (detection) steps all have been integrated and automated in a commercially 
available autosampler. The automation of the entire $\mu$ MISPE-PE-HPLC-FD system for handling samples was the main goal of this chapter. The sampling of an aqueous sample is achieved by drawing a fixed volume of the sample through a stainless steel syringe needle which is packed with $\mu$ MISPE sorbent. The sorbent has predetermined selectivity toward the target analyte molecule. All extraction parameters (such as the filling speed, filling volume, etc.) and the sequence of the programs were direct controlled by the autosampler during $\mu \mathrm{MISPE}-\mathrm{PE} / \mathrm{DPE}-\mathrm{HPLC}-\mathrm{FD}$ analysis. Different sizes of needles were chosen to replace the original one and perform MIPPy/CNTs/QDs- $\mu$ SPE-PE /DPE-HPLC-FD analysis. The results demonstrated that $1.5 "$ (in length) needles were significantly better than those $1.0^{\prime \prime}$ (in length) needles (not shown). Unfortunately, 2.0" needles would be too long to fit in the autosampler. The R.S.D. of recovery $(n=7)$ obtained with 1.5" (in length) MIPPy/CNTs/QDs-modified needle was better than 3\% by using $1.0 \mathrm{~mL}$ of $1.0 \mathrm{ppb}(\mathrm{ng} / \mathrm{mL})$ OTA standard solution as test solution. Due to the closed configuration and simplified operation of the integrated system, loss of analyte during the analytical procedure was avoided and a better recovery was obtained. For example, recovery of 0.5 ppb-OTA spiked Italian red wine using manual MIPPy/CNTs/QDs- $\mu$ SPE-HPLC-FD analysis (analytical procedure was described in section 2.7 ) was only $52 \pm 2 \%$ whereas a recovery of $86 \pm 1 \%$ was obtained with the automated procedure.

The autosampler system combined the characteristics of the automation and selective sample extraction/cleanup. The interface of the MISPE-HPLC system was a MIPPy/CNTs/QDs-modified syringe needle. The developed method facilitated unattended operation and provided selective and enhanced purification of the analyte 
prior to HPLC analysis.

\subsubsection{Preparation of MIPPy/CNTs/QDs-modified stainless steel needles}

A blank stainless steel syringe needle would not bind OTA unless it was modified with a MIPPy film. In this work, MIPPy was electrochemically deposited to encapsulate CNTs and QDs on the inner wall of the needle for OTA binding. This was done according to the procedure reported by $\mathrm{Yu}$ and Lai [40]. The main components of the pre-polymerization mixture were Py, TBAP, EGDMA, CNTs and QDs. The monomer Py, was chosen due to its high affinity for OTA. CNTs provided an extended 3-D framework inside the needle. They suspended well in the pre-polymerization solution and were good conductor of electricity for the electrodeposition of PPy (although it has previously been shown that overlapping of CNTs result in a decrease in their electronic conductance). QDs also have semi-conductive electronic properties that made them a good additional material to increase the total surface area, and hence OTA binding capacity, of the modified needle for $\mu$ MISPE. It was expected that MIPPy would form sheaths around the CNTs and QDs. The process of molecular imprinting involved the interaction of Py monomers and OTA template molecules, whereby equilibrium of non-covalent bonds was created in the pre-polymerization solution. During electropolymerization, a positive potential of $+0.85 \mathrm{~V}$ was applied from the potentiostat onto the stainless steel needle with respect to the $\mathrm{Ag} / \mathrm{AgCl}$ reference electrode. This potential electrochemically oxidized Py, which then reacted with a neighboring monomer to form a chain [115]. The initiated polymerization was allowed to continue propagating for 30-90 min. According to Zhang and Bai, the oxidation potential of PPy is lower than that of the Py monomer [116]. Hence, MIPPy prepared by electrochemical polymerization was probably in its oxidized 
(or cationic) state and carried positive charges within the polymer. TBAP was the doping agent that provided a source of perchlorate anions during the polymerization process. They associated with the positively charged nitrogen atoms on the expanding MIPPy network. This balanced the positive charge of every nitrogen atom that formed as a result of the oxidative electropolymerization. Ethylene glycol dimethacrylate (EGDMA) was used as a cross-linker to provide the structural support that enabled the newly formed MIPPy chains to have a tertiary structure.

\subsubsection{Scanning electron microscopy image}

As previously demonstrated with MIPPy/CNTs, the CNTs were able to provide a rigid 3-D framework inside the stainless steel syringe needle [70]. The microstructure of MIPPy/CNTs/QDs composite films that was formed inside the modified needle (as illustrated in Fig. 4-2(a)) shows strong cohesion between MIPPy, CNTs and QDs. While the outer wall of the needle exhibited a smooth stainless steel surface, the inner wall of the needle was covered by a much rougher deposit of MIPPy-encapsulated CNTs and QDs. Teh et al reported that the presence of CNTs provided more sites for nuclei formation such that there was extensive three-dimensional growth of PPy to attain greater surface roughness [73]. Another SEM image of MIPPy/CNTs/QDs-modified surface was obtained under high magnification of 15,000X (Fig. 4-2 (b)). Compared to the substrate without modification (Fig. 4-2 c), it reveals lots of cauliflower like nodules (up to $1 \mu \mathrm{m}$ in size). This indicates that modification with MIPPy/CNTs/QDs dramatically increased the surface area of the stainless steel needle. We attribute this increase of surface area to the successful encapsulation of CNTs and QDs by MIPPy film which served as macromolecular glue. 


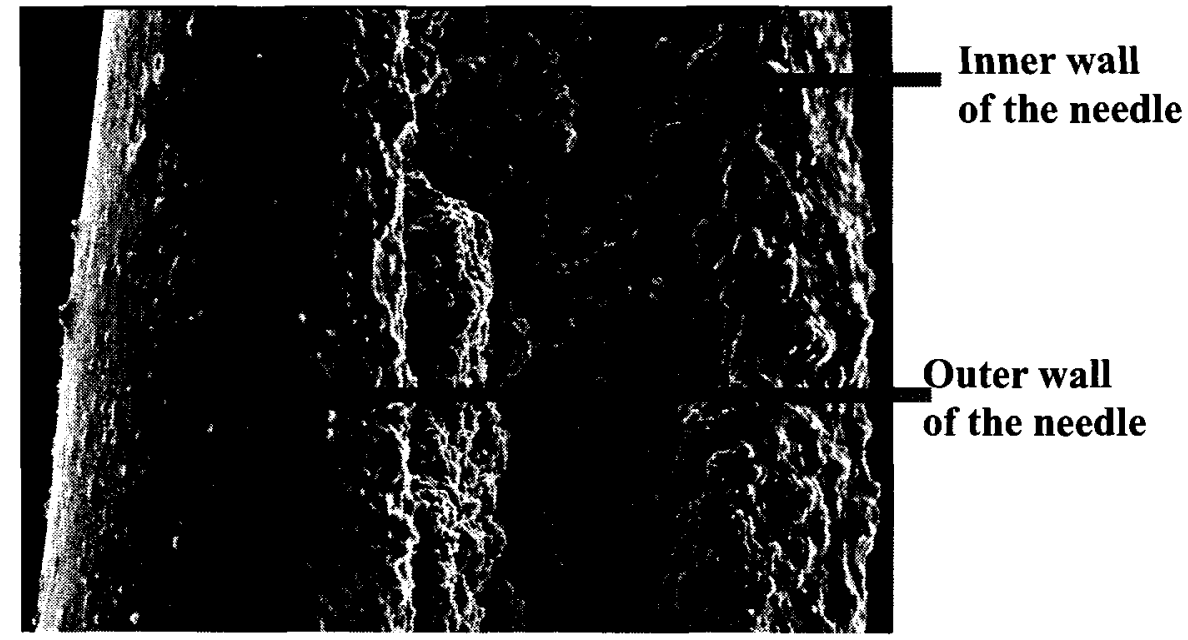

(a)

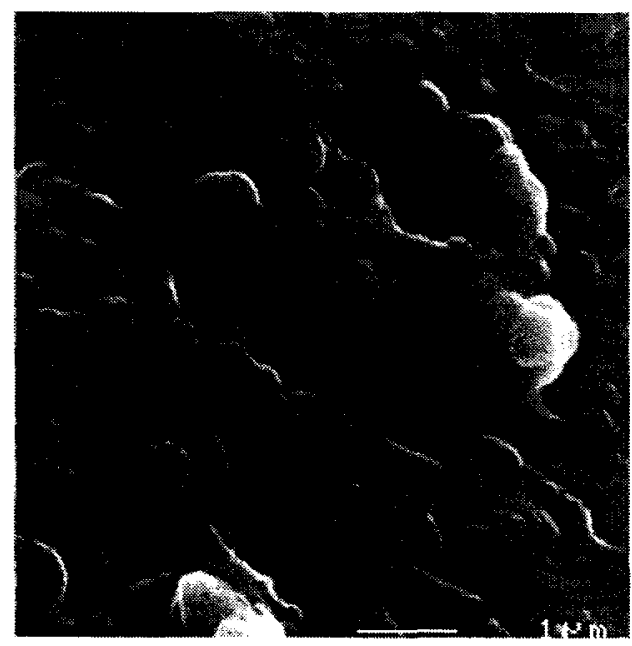

(b)

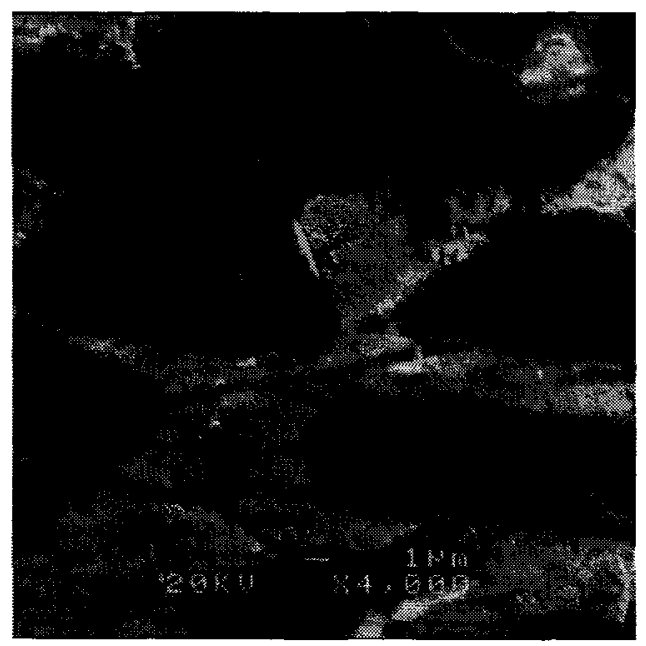

(c)

Fig. 4-2 SEM images of a MIPPy/CNTs/QDs-modified stainless steel syringe needle tip (a) at a low resolution; (b) at a high resolution; (c) stainless steel surface before modification

After the electropolymerization, all template OTA molecules were removed from the MIPPy/CNTs/QDs network, creating the appropriate cavities specifically tailored for rebinding OTA. Every cavity would have the ideal size, shape, and orientation of functional groups to optimize rebinding interactions. The atomic force microscope (AFM) would ideally be suited for characterizing nano-particles and -structures. It offers the 
capability of 3D visualization and qualitative/quantitative information on many physical properties including size, morphology, surface texture and roughness. Statistical information (including size, surface area, and volume distributions) can be determined as well. A wide range of particle sizes can be characterized in the same scan (from $1 \mathrm{~nm}$ to $8 \mu \mathrm{m})$. In addition, the AFM can characterize nanoparticles in multiple mediums including ambient air, controlled environments, and even liquid dispersions. Unfortunately, stainless steel needles (of the type used in this work) were not suited for AFM analysis because they lacked a flat surface in an accessible area.

\subsubsection{Optimization of template OTA concentration in pre-polymerization mixture}

Pre-electropolymerization mixture with $100 \mu \mathrm{M}$ OTA has shown specific affinity toward OTA in red wine [61]. More studies are needed to further investigate the recognition mechanism.

For non-covalent molecular imprinting, several factors have been identified that are likely to affect the creation of the recognition site, such as thermodynamic factors, the number and the type of interaction sites of the monomer and the template, the molar relationship between the functional monomer and template, the monomer-template rigidity and the template shape [117]. In this thesis study, the effect of different molar ratios of functional monomer (Py) and template (OTA) on the recovery of OTA by using the MIPPy/CNTs/QDs-modified needle was examined as greatly facilitated by the automated HPLC-FD system. The concentration of Py was fixed while the OTA concentration was varied to obtain different monomer to template $(\mathrm{M} / \mathrm{T})$ ratios. It was observed that the recovery of $1.0 \mathrm{ng}$ OTA $(=1.0 \mathrm{~mL} \times 1.0 \mathrm{ppb}$ standard solution $)$ was enhanced with increasing template concentration. When the template concentration was 
$100 \mu \mathrm{M}(\mathrm{M} / \mathrm{T}=1000: 1)$, the recovery of $1.0 \mathrm{ng}$ OTA $(=1.0 \mathrm{~mL} \times 1.0 \mathrm{ppb}$ standard solution) was only $76 \pm 2 \%$; while the recovery dramatically increased to $95 \pm 3 \%$ when the template concentration was increased up to $300 \mu \mathrm{M}(\mathrm{M} / \mathrm{T}=333: 1)$ as shown in Fig. 4-3. These results clearly demonstrated that with an increase of available binding sites on the MIPPy/CNTs/QDs, the extraction efficiency (in terms of recovery) was enhanced.

It is worth noting that even a much lower concentration of template was employed in this work than the normal ratio of $4: 1(\mathrm{M} / \mathrm{T})$ generally used for non-covalent MIP

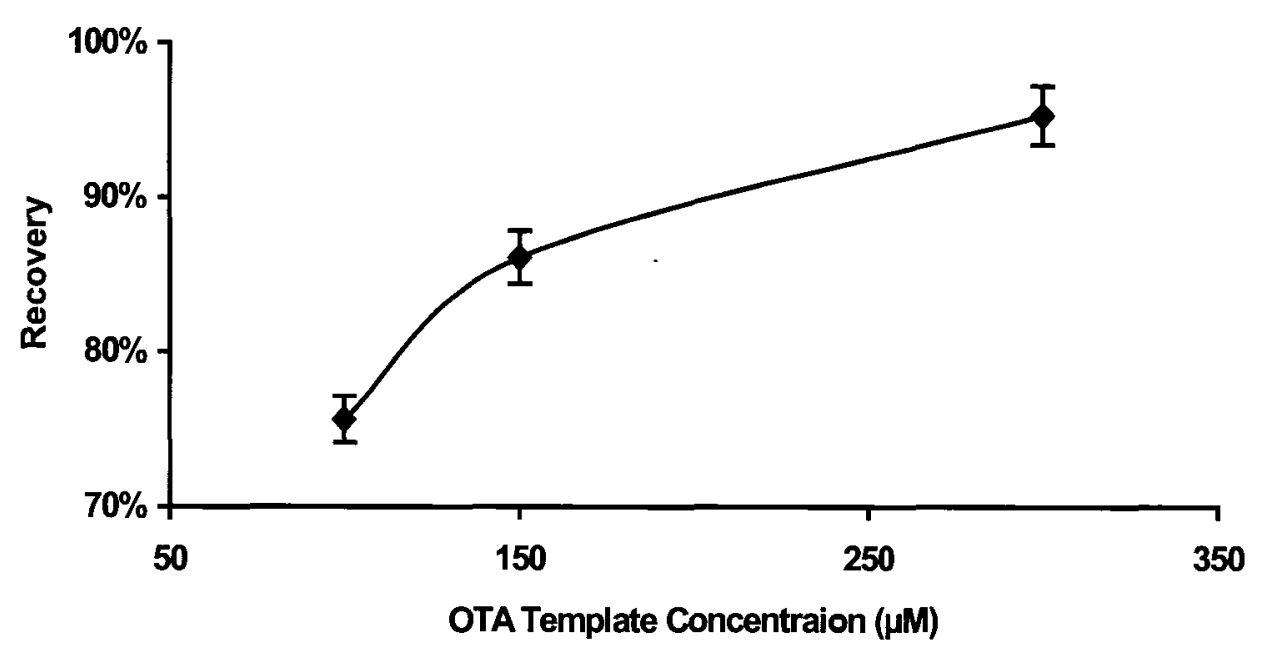

Fig. 4-3 Effect of template OTA concentration in pre-polymerization mixture on $\mu$ MISPE efficiency of MIPPy/CNTs/QDs-modified needle

systems $[1,12]$, the binding capacity of the MIPPy/CNTs/QDs-modified needle was still good enough for determination of OTA contamination up to the $2.0 \mu \mathrm{g} / \mathrm{L}$ level (as shown in Fig. 4-6). Note that $2.0 \mu \mathrm{g} / \mathrm{L}(=2.0 \mathrm{ppb})$ is the maximum allowable concentration of OTA in wine set by European Union [55].

Some studies have demonstrated that the concentration of the template in the pre-polymerization mixture can be reduced dramatically without major losses of quality 
or performance of the resulting MIPs. With M/T ratio of 500:1 or even 5000:1, MIPs could still be produced to bind significantly more analyte than the control polymer (non-imprinted polymers) $[1,12]$. The benefits of using low template concentration in this study are easy removal of template and decreased cost since OTA are relative expensive.

\subsubsection{Effect of analyte OTA concentration on recovery of automated MIPPy /CNTs/QDs- $\mu$ SPE-PE-HPLC-FD}

The effect of analyte OTA concentration on recovery of automated MIPPy/CNTs/QDs- $\mu$ SPE-PE-HPLC-FD was investigated over the OTA concentration range from $0.02-3.0 \mathrm{ng} / \mathrm{mL}(0.02-3.0 \mathrm{ng}$ OTA). As show in Fig. $4-4$, the OTA concentration has no significant effect on recovery. Over the tested concentration range, $80 \%-101 \%$ recovery could be achieved with R.S.D. better than $6 \%$. These results show that the OTA imprinted MIPPy/CNTs/QDs behaves well to recognize OTA molecules at the various concentration levels.

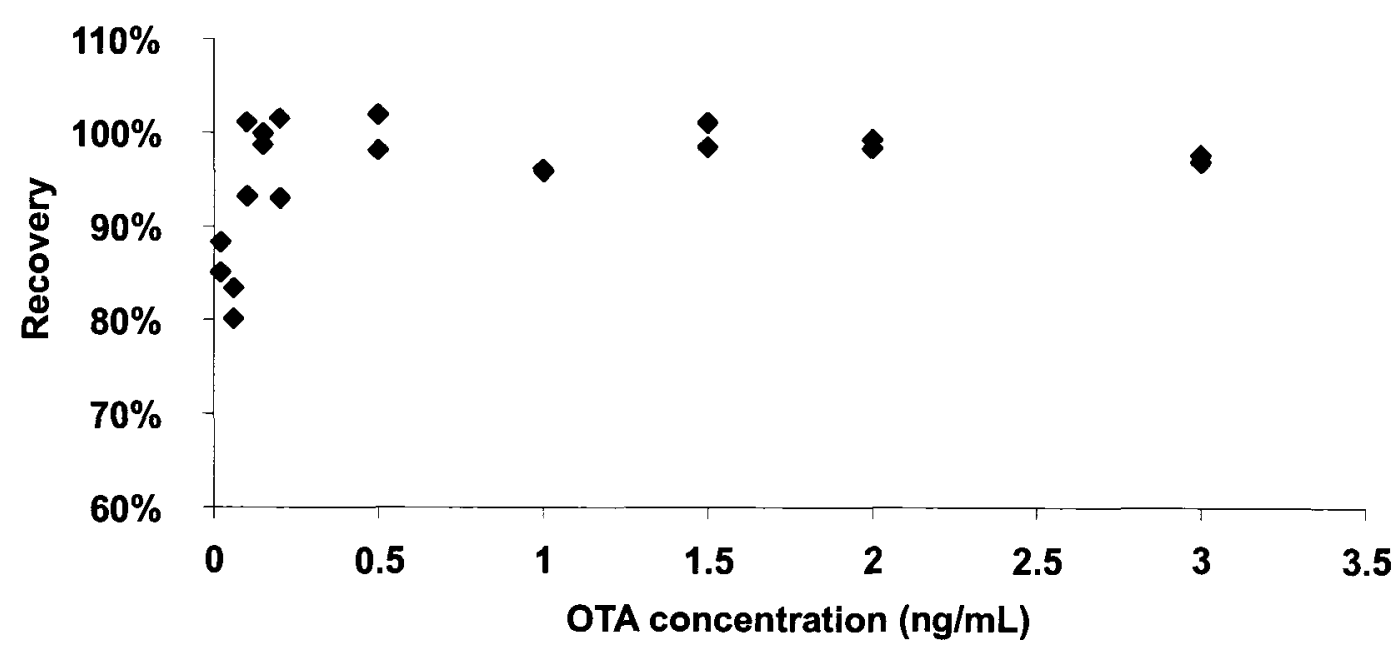

Fig. 4-4 Effect of OTA analyte concentration on recovery of automated MIPPy/CNTs/QDs- $\mu$ SPE-PE-HPLC-FD 


\subsubsection{Standard calibration curve for automated MIPPy/CNTs/QDs- $\mu$ SPE-PE -HPLC-FD analysis}

A calibration curve was constructed from the peak area results obtained by MIPPy/CNTs/QDs- $\mu$ SPE-PE-HPLC-FD analysis of $1.0-\mathrm{mL}$ OTA standard solutions using the automated system. As shown in Fig. 4-5, within the OTA concentration range from 0.02-3.0 $\mathrm{ppb}(\mathrm{ng} / \mathrm{mL})$, the MIPPy/CNTs/QDs- $\mu$ SPE-PE-HPLC-FD peak area was linearly $\left(\mathrm{R}_{2}=0.9962\right)$ related to OTA concentration (or mass of OTA loaded on the MIPPy/CNTs/QDs-modified needle). The precision of MIPPy/CNTs/QDs- $\mu$ SPE-HPLC-

FD analysis was good, with a relative standard deviation under $6 \%$. Using $1.0 \mathrm{~mL}$ of standard solution for pre-concentration, it was possible to determine OTA down to a limit of detection of $0.02 \mathrm{ppb}\left(\mathrm{LOD}=3 \sigma_{\mathrm{B}} / \mathrm{b}, \mathrm{n}=7\right.$, R.S.D. $\left.=3 \%\right)$, or a limit of quantification of $0.06 \mathrm{ppb}\left(\mathrm{LOQ}=10 \sigma_{\mathrm{B}} / \mathrm{b}, \mathrm{n}=7\right.$, R.S.D. $\left.=3 \%\right) . \quad$ The time for automated sample processing by MIPPy/CNTs/QDs- $\mu$ SPE-PE-HPLC-FD was $16 \mathrm{~min}$, which is significantly shorter than the 60 min typically required with the use of IAC columns [61].

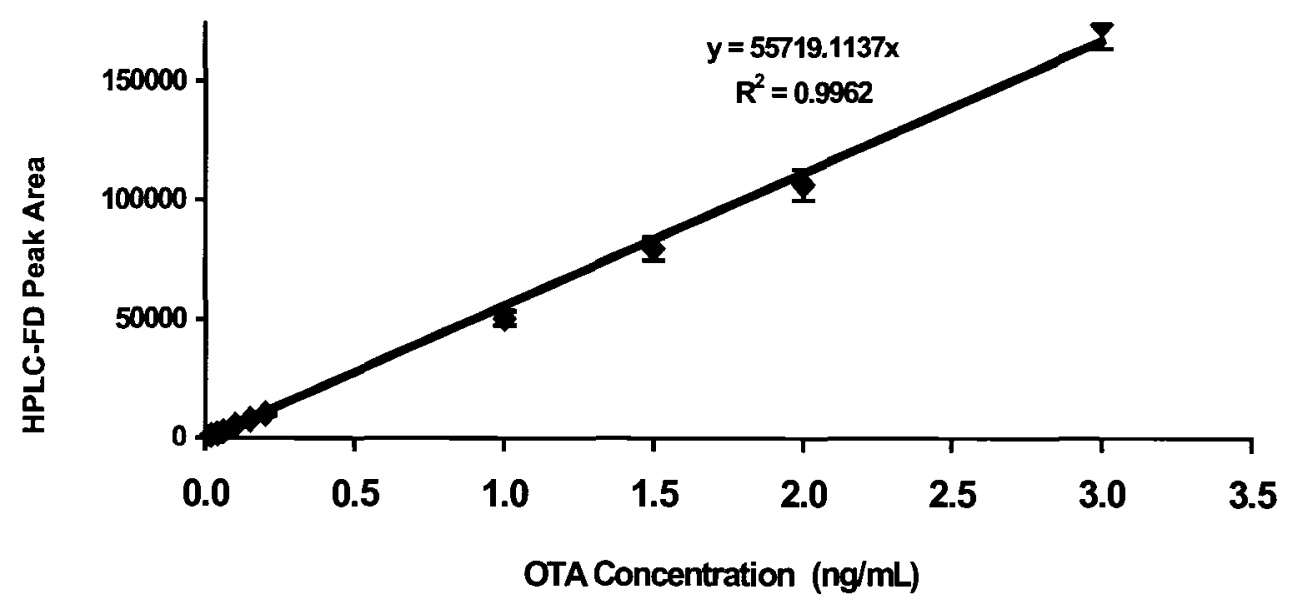

Fig. 4-5 Standard calibration curve of automated MIPPy/CNTs/QDs- $\mu$ SPE-PEHPLC-FD 


\subsubsection{Measurement of total surface area by pulsed amperometry}

A defining feature of CNTs and QDs is their large specific surface areas (SSA) [118], which is the surface area per unit mass. Specific surface area can indicate the average particle size, but not particle size range or distribution. Traditionally, gas absorption techniques such as Brunauer-Emmett-Teller (BET) analysis would be used to determine SSA. Instead of $\mathrm{N}_{2}$ single point BET, electrochemical analysis was used in this work to measure the total surface area of each MIPPy/CNTs/QDs-modified needle. Conductive PPy, MIPPy/CNT and MIPPy/CNTs/QDs, in 0.1 M Na2 $\mathrm{SO}_{4}$ electrolyte solution, allowed for amperometric measurement of the total surface area when an applied potential was pulsed between $-0.2 \mathrm{~V}$ and $+0.4 \mathrm{~V}$ versus the $\mathrm{Ag} / \mathrm{AgCl}$ reference electrode [114]. $\mathrm{A}$ current signal was generated when the electrolyte was run through the MIPPy/CNTs/QDs modified needle that served as a working electrode. The current was recorded over a short period of time for signal averaging. The average pulsed amperometry detection (PAD) current signal was compared with that obtained for a MIPPy-modified needle (without any encapsulated CNTs or QDs), as summarized in Fig. 4-6a. It became obvious that the encapsulation of CNTs/QDs increased the total surface area significantly. A PPy-modified needle served as the control for surface area measurement and for recovery analysis (recovery $=38.5 \%$ ). Further calibration of the PAD current versus the nominal surface area was made possible with the use of several commercially-available stainless steel syringe needles of different gauges and lengths, as presented in Fig. 4-6b. 


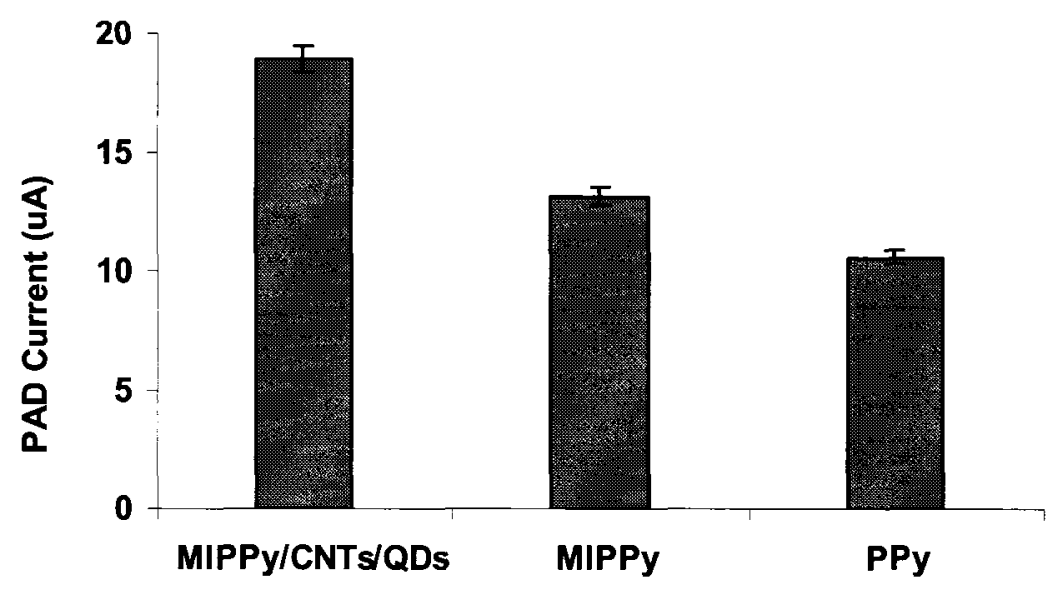

Fig. 4-6a Comparison of PAD currents measured for MIPPy/CNTs/QDs-modified needle, MIPPy-modified needle, and PPy-modified needle.

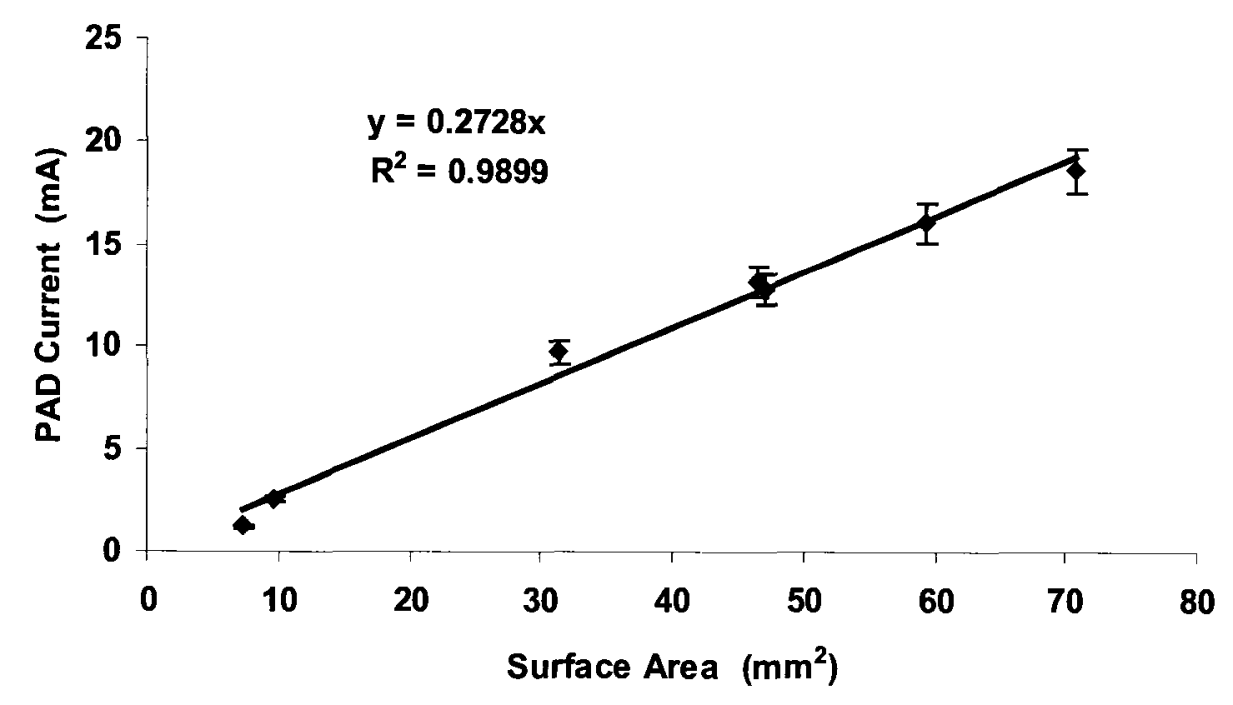

Fig. 4-6b Calibration of PAD current versus nominal surface area of commercially available stainless steel syringe needles of different gauges and lengths 


\subsubsection{Effect of surface area over the recovery}

MIPPy/CNTs and MIPPy/CNTs/QDs-modified needles with different surface areas were obtained by modifying needles with different lengths and gauges. The surface area was measured by pulsed amperometry. From the plot of recovery versus surface area (Fig. 4-7), we can see that with the increase of the surface area of MIPPy/CNTs and MIPPy/CNTs/QDs-modified needles, better recoveries could be obtained.

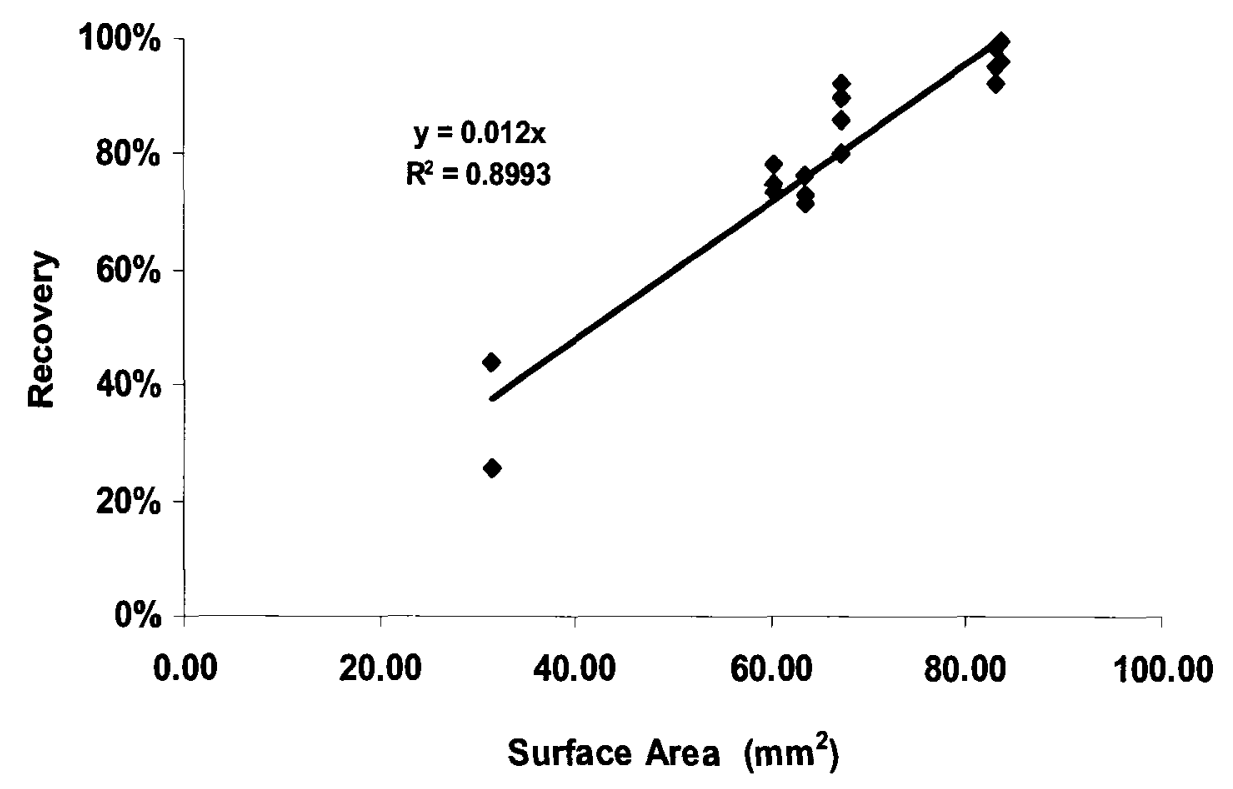

Fig. 4-7 recovery of OTA from $1 \mathrm{ppb}(\mathrm{ng} / \mathrm{mL})$ OTA standard solution by MIPPy/CNTs and MIPPy/CNTs/QDs-modified needles of different total surface areas

\subsubsection{Automated $\mu$ MISPE-PE-HPLC-FD analysis of red wine}

Red wines are composed of a complex mixture of water, phenolic compounds, organic acids, alcohols, and residual sugars [118]. Some study revealed that the chemical composition of wine is containing more than 500 different compounds in a wide 
range of concentrations [119]. Because of many interfering compounds that exist in red wine, one general problem in the HPLC-FD determination of OTA in red wine samples would be the interference from florescent matrix components (polyphenols). As shown in Fig. 4-8(b), the large peaks from different components in the Italian red wine matrix made quantitative interpretation very difficult in direct analysis of the 0.5 ppb-OTA-spiked red wine. The discrimination ability should be greatly enhanced, if these components are selectively removed before introduction of red wine sample to the HPLC instrument for analysis. Unfortunately, sample cleanup by commercial SPE cartridge tended to remove both OTA and matrix components indiscriminately. Varelis et al even reported overload of the detector caused by the co-extracted polyphenols [120]. In this thesis study, the MIPPy/CNTs and MIPPy/CNTs/QDs-modified stainless steel needles were employed to pre-concentrate OTA from red wine matrix, thereby cleaning up the sample. Relatively clean chromatograms were obtained from both MIPPy/CNTs- $\mu$ SPE-PE-HPLC-FD analysis (Fig. 4-8(c)) and MIPPy/CNTs/QDs$\mu$ SPE-PE-HPLC-FD analysis (Fig. 4-8(d)) after enrichment of OTA from $1.0 \mathrm{~mL}$ of 0.5 ppb-OTA spike red wine. The results demonstrated that MIPPy/CNTs- $\mu$ SPE and MIPPy/CNTs/QDs- $\mu$ SPE were able to resolve OTA peaks from the red wine matrix components. Potential interference from the matrix components was thus greatly decreased. The chromatograms also show that MIPPy/CNTs/QDs, with its larger total surface area (surface area $=83.5 \mathrm{~mm}^{2}$ measured by pulsed amperometry), extracted both more OTA and more matrix components than MIPPy/CNTs (surface area $=60.4 \mathrm{~mm}^{2}$ measured by pulsed amperometry). 


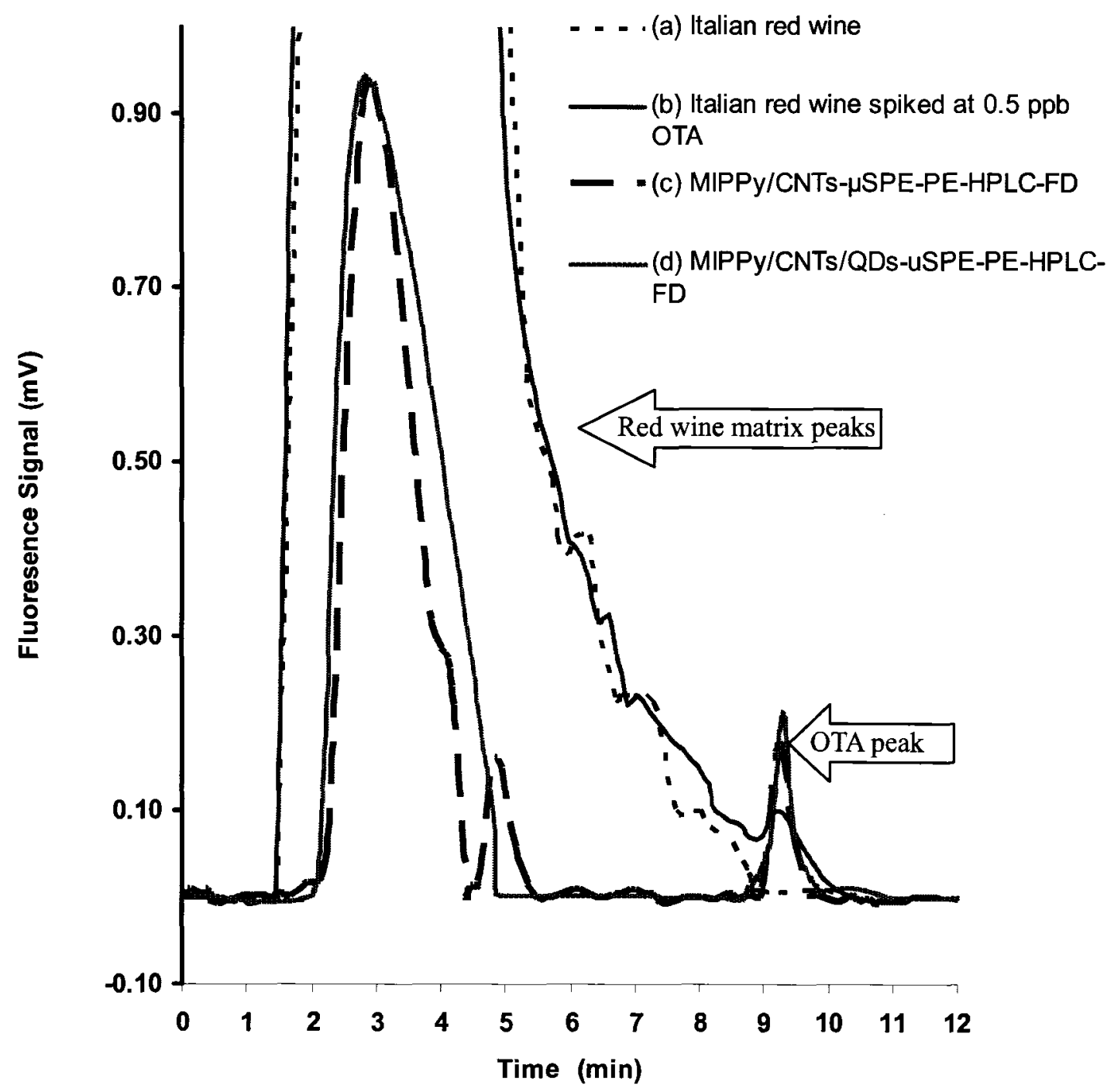

Fig. 4-8 (a) Direct HPLC-FD analysis of the Italian red wine

(b) Direct HPLC-FD analysis of the Italian red wine spiked at 0.5 ppb OTA

(c) MIPPy/CNTs- $\mu$ SPE-PE-HPLC-FD analysis after enrichment of OTA from $1.0 \mathrm{~mL}$ of the Italian red wine spiked at $0.5 \mathrm{ppb}$ OTA

(d) MIPPy/CNTs/QDs- $\mu$ SPE-PE-HPLC-FD analysis after enrichment of OTA from $1.0 \mathrm{~mL}$ of the Italian red wine spiked at $0.5 \mathrm{ppb}$ OTA 


\subsubsection{Improvement in recovery of OTA in red wine analysis}

The recovery results of the Italian red wine matrix components and OTA from PPy/CNTs/QDs, MIPPy/CNTs and MIPPy/CNTs/QDs are compared in Fig. 4-9. Recoveries of red wind matrix components were calculated by comparing the red wind matrix peak areas from direct HPLC analysis of spiked samples and after $\mu$ MISPE enrichment of spiked samples with the modified needles. Note that the MIPPy/CNTs/QDs-modified needle afforded the highest OTA recovery of $86 \pm 1 \%$ and a modest $10.2 \pm 3 \%$ recovery of red wine matrix components (same as the PPy/CNTs/QDs modified needle) due to non-specific binding. Using the ratio of recovery results between the red wine matrix components and the OTA as an alternative indicator of selectivity (data not shown), it strongly suggests that better selectivity toward the target OTA molecules was the benefit of molecularly imprinting done for the two MIPPy modified needles.

Comparing the recovery of OTA measured for a MIPPy/CNTs-modified needle (without any encapsulated QDs) and MIPPy/CNTs/QDs-modified needle, it suggested that the QDs enhanced (by 1.4-fold) the total surface area available for $\mu$ MISPE inside the modified needle. Such a significant improvement was attained at the price of an amenable increase of back pressure during the $\mu$ MISPE step, which was well within the operational limits of the autosampler itself. Consequently, the detection limit was improved by 1.5 -fold to reach a new low level of $0.02 \mathrm{ng} / \mathrm{mL}$ OTA in red wine analysis. 


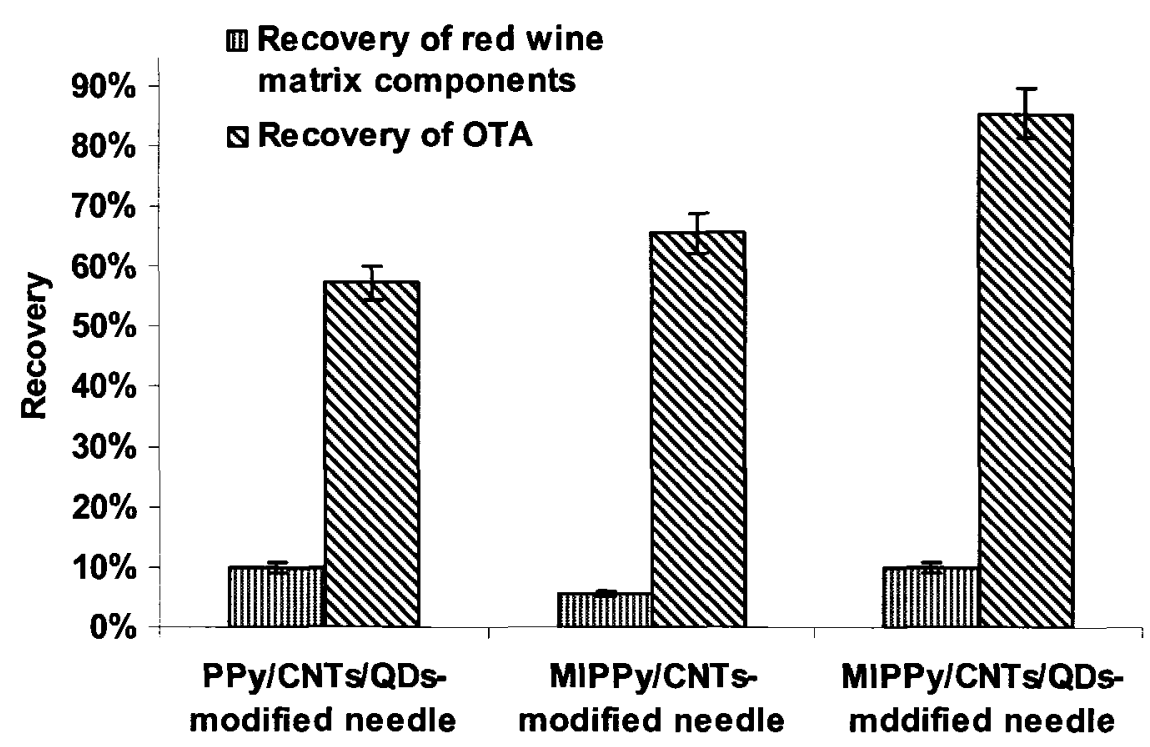

Fig. 4-9 Recoveries of red wind matrix components and OTA using MIPPy/CNTsand MIPPy/CNTs/QDs-modified needles. Data from a non-imprinted PPy/CNTs/QDs-modified needle are included for comparison.

\subsubsection{Elimination of non-specific binding of red wine matrix components with} differential pulsed elution

Previously the concept underpinning MIP theory was often exemplified by the lock-and-key model [15], based on enzyme-substrate interactions in biochemistry. X-ray diffraction later refuted this concept, showing that enzymes sustain a more flexible structure than synthetic MIPs. Nevertheless, MIPs are more thermodynamically stable and cost effective than their biological counterparts. They can be relatively easy to make and can be stored for many years without losing their structural integrity. One question we asked ourselves was: Is MIPPy/CNTs/QDs truly a new MIP in this nano-structured device (of modified syringe needle)? To investigate this issue, differential pulsed elution (DPE) was employed, which had been described in our previous reports [47, 48, 121]. 
DPE involved intermediate wash steps that would remove much of the weakly bound wine matrix components from the MIPPy/CNTs/QDs modified needle. In our previous work on MIPPy/CNTs-modified stainless steel frits, several organic solvents were evaluated for their DPE effectiveness by using $100 \mu \mathrm{L}$ of each solvent to wash the frit before PE-HPLC-FD analysis [76]. In this thesis study, DDW/85\% $\mathrm{H}_{3} \mathrm{PO}_{4}(60: 1$ by volume) and 1.0 M HCl were investigated for their DPE efficiency, by using $200-\mu \mathrm{L}$ aliquots of each solvent to wash the MIPPy/CNTs/QDs-modified needle before the final pulsed elution (FPD) with $2 \%$ TEA. Ideally, DPE must eliminate all the wine matrix components quantitatively while keeping a good amount of OTA in the needle for subsequent HPLC-FD analysis. Fig. 4-10 resulted from the MIPPy/CNTs/QDs-DPE-HPLC-FD analysis of the Italian and Canadian red wine samples spiked at $0.5 \mathrm{ppb}(\mathrm{ng} / \mathrm{mL})$ OTA level. As it be seen in Fig. 4-10 (a), DDW/85\% $\mathrm{H}_{3} \mathrm{PO}_{4}$ (by volume) was bad to elute red wine matrix peak but good enough to wash $40 \%$ of the bound analyte OTA from the MIPPy/CNTs/QDs (in the first three DPEs, total volulme $=600 \mu \mathrm{L}$ ). It shows in Fig. 4-10 (b) that weakly bound wine matrix components were totally eluted during the first three DPEs with $1.0 \mathrm{M} \mathrm{HCl}$ (total volume $=600 \mu \mathrm{L}$ ), while the strongly bound OTA was kept on the MIPPy/CNTs/QDs-modified needle. This new finding looked promising because a clean chromatogram (as in Fig. 4-14) was obtained without any interference of red wine components. The OTA peak (retention time $=6.4 \mathrm{~min}$ ) was totally resolved down to the baseline. Now we know for sure that this MIPPy on CNTs/QDs was truly a new MIP! 


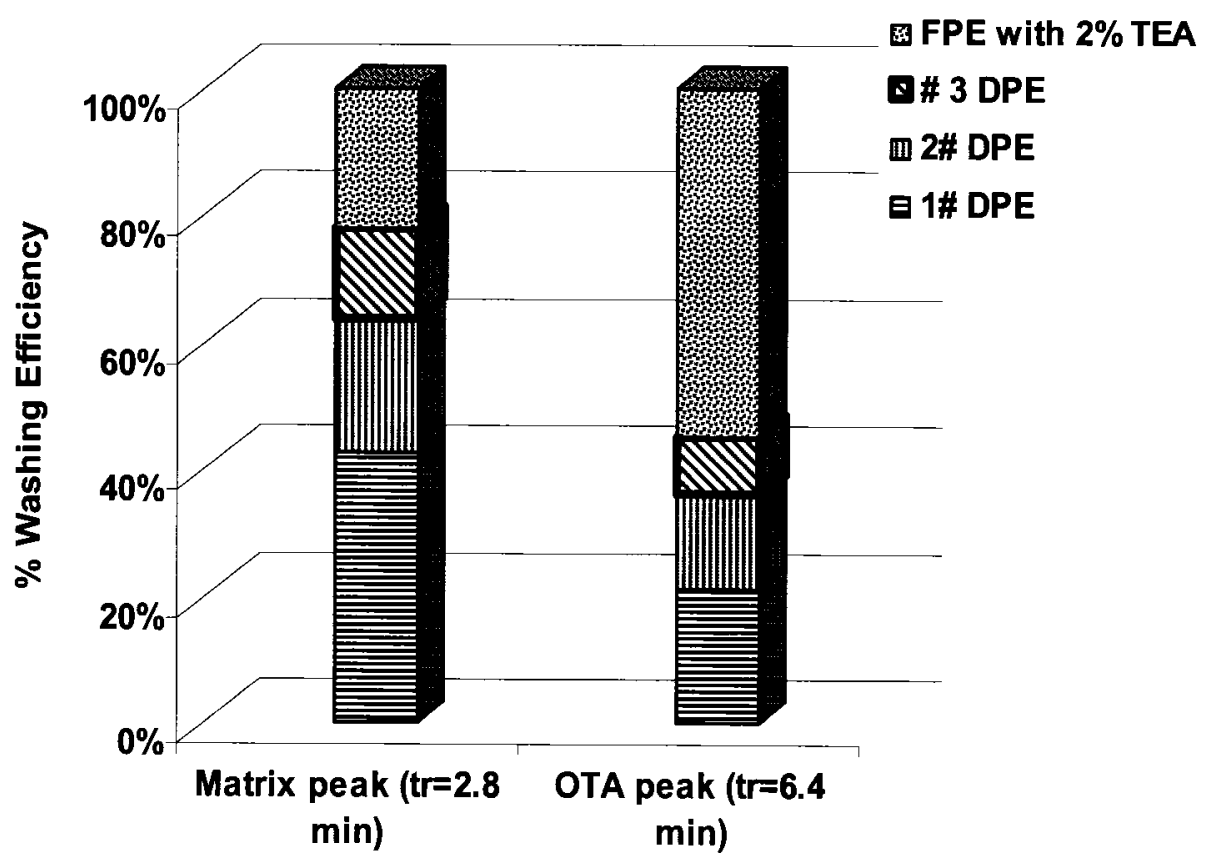

(a)

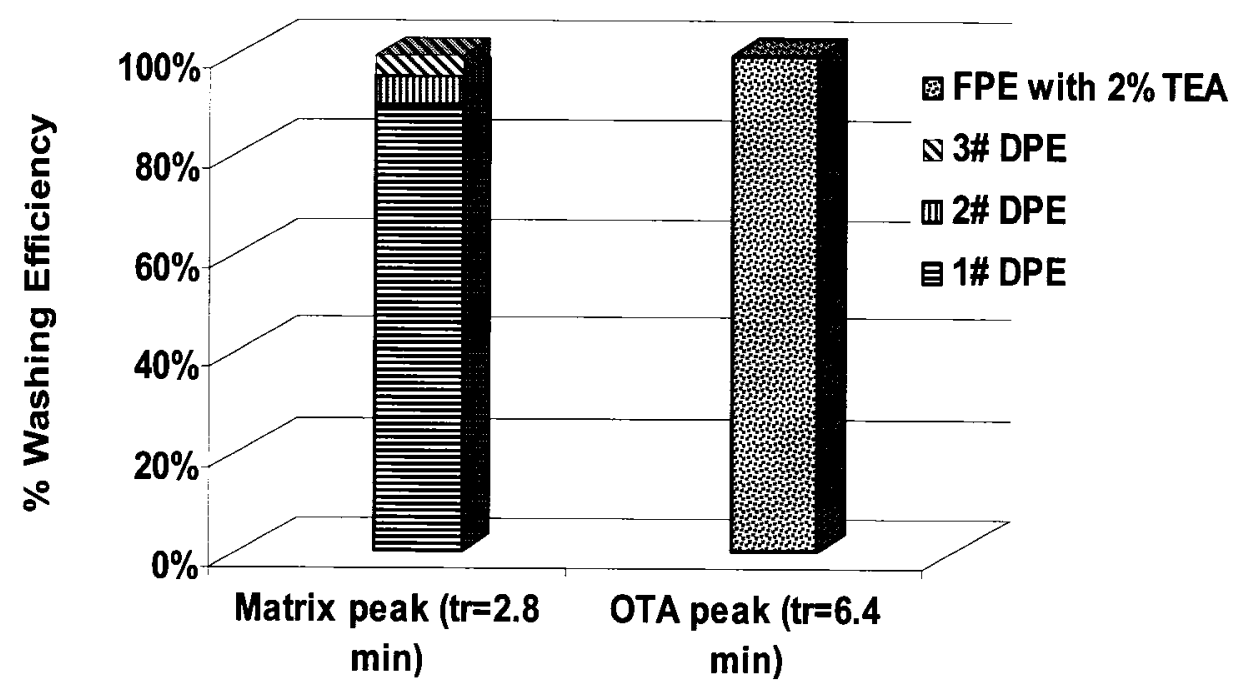

(b)

Fig. 4-10 Evaluation of different solvents for their DPE effectiveness on OTA, by using $600 \mu \mathrm{L}$ of each solvent (in three times) for washing the MIPPy/CNTs/QDs-modified needle prior to PE-HPLC-FLD analysis. (a) DDW/85\% $\mathrm{H}_{3} \mathrm{PO}_{4}$ (60:1 by volume); (b) $1.0 \mathrm{M} \mathrm{HCl}$. 


\subsubsection{Effect of different red wine matrices}

In order to investigate the effect of different red wine samples on the performance of MIPPy/CNTs/QDs-modified $\mu$ SPE device, different wine samples were analyzed using external calibration and standard additions. No detectable OTA was found in the Italian and Canadian red wine samples. From the chromatograms obtained from direct HPLC-FD analysis, it can be seen in Fig. 4-11(a) and (b) that, despite the very different shapes and intensities of matrix peaks of Italian and Canadian red wine samples, they all interfere with the accurate quantification of OTA. These fluorescent red wine components are believed to be polyphenols present at relatively high concentrations in red wine. They include phenolic aldehydes, acids and flavonoids. Phenolic compounds, as an important group of substances, contribute to several sensorial characteristics of red wine, such as colour, flavour, astringency $[119,122]$. The chemical structures of several common phenolic compounds in red wine are shown in Fig. 4-12 [119, 123]. Since the types and concentrations of polyphenols in wine vary depending on a wide variety of factors (for example, grape variety and ripening, atmospheric conditions, vine cultivation practices and production process) [124], it is very difficult to identify which compounds interfere with OTA determination in each red wine analysis. Our attempt to identify these interference compounds in Italian red wine sample failed due to the lack of such compounds in our lab and also because of the difficulty to mimic the actual red wine. Despite the very different matrix peaks of these two brands of red wines, the MIPPy/CNTs/QDs-modified $\mu$ SPE device shows consistent recovery for spiked samples $(86 \pm 1 \%$ for Italian red wine and $90 \pm 1 \%$ for Canadian red wine). As shown in Fig. 4-11(b) and (c), after cleanup with the MIPPy/CNTs/QDs-modified $\mu$ SPE device, relatively clean chromatograms could be 
obtained for both red wine samples. The results demonstrated that the MIPPy/CNTs/QDs-modified $\mu$ SPE device can be applied on different red wines in determination of OTA. DPE experiments with these two wines (Fig. 4-13(a) and (b)) exhibited that the procedure developed in this work can also be applied to different red wine samples. The recoveries of spiked Italian red wind and Canadian red wine obtained with FPE after three DPEs were $80 \pm 1 \%$ and $86 \pm 1 \%$, respectively.

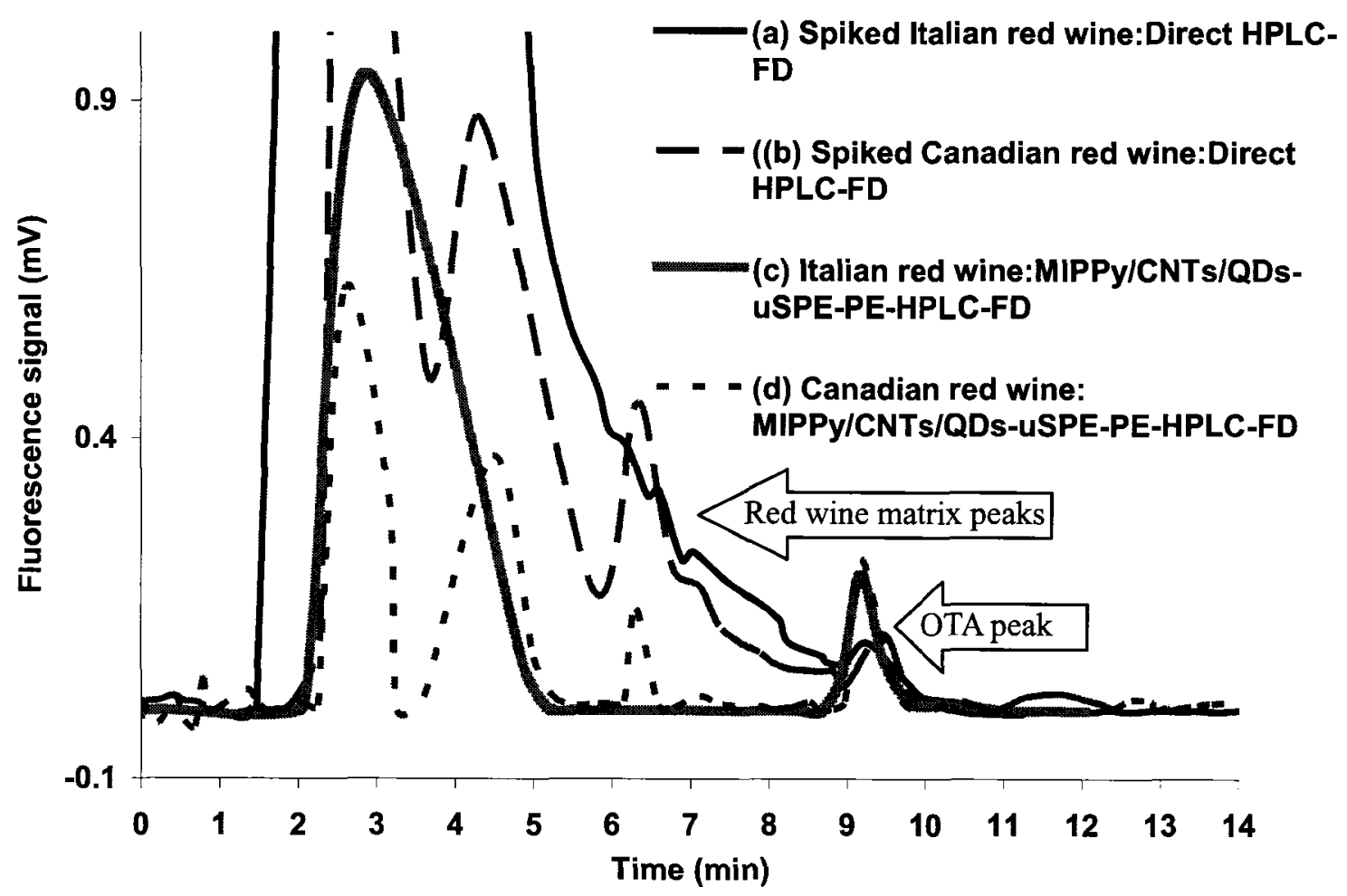

Fig. 4-11 (a) Direct HPLC-FD analysis of 0.5 ppb spiked Italian red wine;

(b) Direct HPLC-FD analysis of 0.5 ppb spiked Canadian red wine;

(c) $\mathrm{MIPPy} / \mathrm{CNTs} / \mathrm{QDs}-\mu \mathrm{SPE}-\mathrm{PE}-\mathrm{HPLC}-F D$ analysis after enrichment of OTA from $1.0 \mathrm{~mL}$ of Italian red wine spiked at $0.5 \mathrm{ppb}$ OTA;

(d)MIPPy/CNTs/QDs- $\mu$ SPE-PE-HPLC-FD analysis after enrichment of OTA from $1.0 \mathrm{~mL}$ of Canadian red wine spiked at $0.5 \mathrm{ppb}$ OTA. 
<smiles>O=C(O)c1cc(O)c(O)c(O)c1</smiles>

Gallic acid

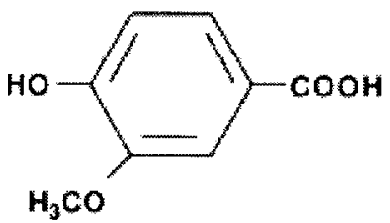

Vanillic acid<smiles>O=C(O)c1ccc(O)c(O)c1</smiles>

Protocatechuic acid<smiles>COc1cc(C(=O)O)cc(OC)c1O</smiles>

Syringic acid (n)<smiles>[Li]</smiles><smiles>O=C(O)c1ccc(O)cc1</smiles>

p-Hydroxybenzolc acid

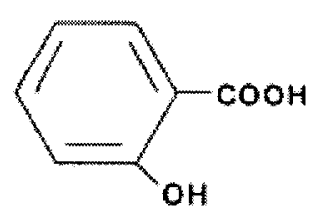

salicylic acid 


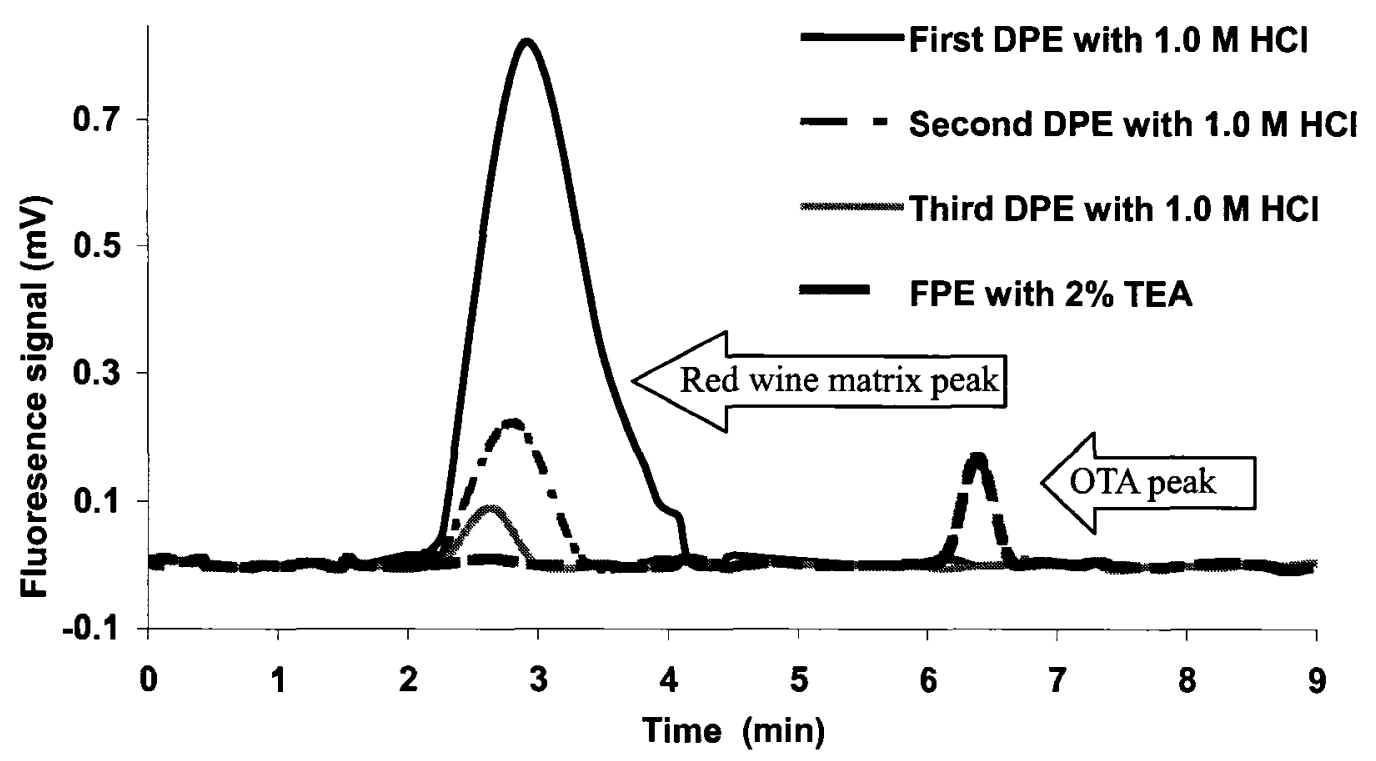

(a)

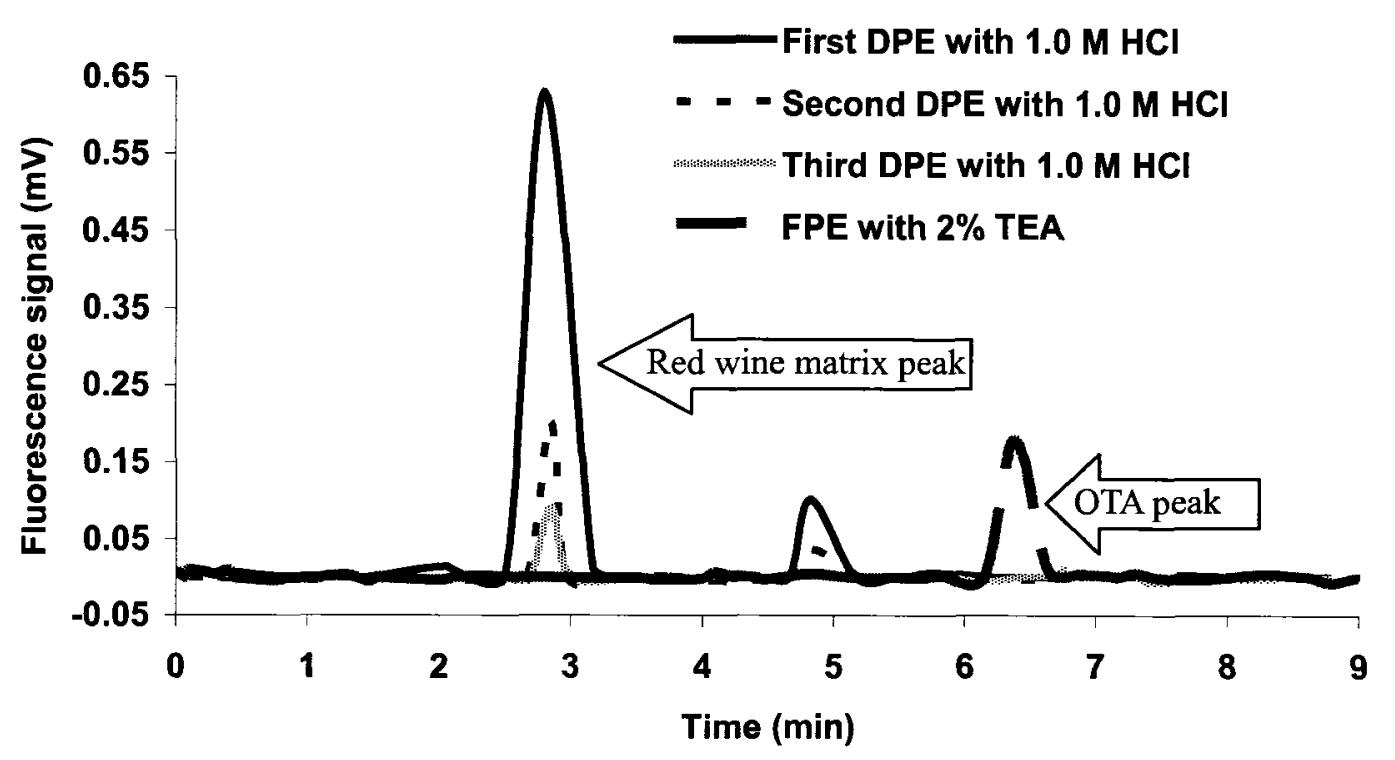

(b)

Fig. 4-13 HPLC-FD analysis of differential pulsed elution (DPE) of red wine matrix components with $3 \times 200 \mu \mathrm{L}$ of $1.0 \mathrm{M} \mathrm{HCl}$, and final pulsed elution (FPE) of OTA with 1 x $200 \mu \mathrm{L}$ of $2 \%$ TEA. (a) Italian red wine; (b) Canadian red wine 


\subsection{Conclusion}

A new $\mu$ MISPE device has been made and an atutomated $\mu$ MISPE-HPLC method for determination of OTA in red wine has been developed. The new $\mu$ MISPE device used MIPPy-encapsulated CNTs and QDs as the sorbent to attain a significantly larger total surface area. The MIPPy/CNTs/QDs composite was grown using electrochemical polymerization, in which the conductive polymer, CNTs and QDs were simultaneously deposited along the inner wall of the stainless steel syringe needle to form a three-dimensional network. A DPE process was developed which exhibited adequate effectiveness to remove the interfering components of a complex red wine matrix and a significant enhancement of binding capacity (and recovery) for OTA as well as. The automation of $\mu$ MISPE-HPLC procedure was established by coupling the MIPPy/CNTs/QDs modified needle with the commercially available HPLC autosampler. The automation of the $\mu$ MISPE-HPLC procedure afforded rapid determination of ultratrace OTA in red wine with good precision and low contamination due to the closed configuration of the system. It is commonly believed that both the acceptance and the range of potential applications for a new technique with automation can be significantly promoted. These newly developed MIPPy/CNTs/QDs-modified needles will be evaluated for the $\mu$ SPE processing of various food samples, prior to their reverse-phase or size exclusion liquid chromatography for the control of food quality and the monitoring of food safety. With further development of nanomaterials to open new avenues for sensing and instrumentation research, we will truly be on the verge of a major technological breakthrough that can have far-reaching impacts to all aspects of food science and engineering. 


\section{CHAPTER 5}

\section{Conclusions}


This research developed a new technique for the synthesis of OTA imprinted PPy based nano-composites as $\mu$ MISPE sorbents and designed a fully automated analytical method for determination of ultratrace OTA in red wine samples. The MISPE sorbent was grown by using electrochemical polymerization, in which the conductive polymer (polypyrrole), carbon nanotubes (CNTs) and quantum dots (QDs) were simultaneously deposited along the inner wall of the stainless steel syringe needle to form a three-dimensional network. Taking advantage of the nano-composite based MISPE sorbent, a new configuration of automated $\mu$ MISPE coupled to HPLC-FD was designed, which integrated sampling (extraction)/pulsed elution/injection (detection) into one closed system. The fully automated and validated method exhibited a significant enhancement of binding capacity (and recovery) for OTA, as well as adequate effectiveness to remove the interfering components of a complex red wine matrix.

The in-situ electropolymerization method demonstrated in this study did not need any subsequent time-consuming steps of grinding, sieving and packing. The conducting property of CNTs and QDs was utilized to form nano-composites of MIPPy/CNTs and MIPPy/CNTs/QDs which served as a molecularly imprinted polymeric matrix and scaffold of packing materials inside a stainless steel syringe needle. Due to the much large surface area provided by the nanostructure of MIPPy/CNTs/QDs, the binding sites are more accessible. This enabled faster diffusion and mass transfer of analyte OTA molecules to the binding cavities. Hence the re-binding process of analyte is faster and more efficient during SPE. To analyze trace OTA in red wine, only $1.0 \mathrm{~mL}$ of red wine sample was required and the total analysis time only takes less than 20 minutes. The synthetic method developed in this thesis research opens a new strategy that combines the 
unique characteristics of nanomaterials and molecular imprinting technique. The successful application of nano-sized MISPE sorbent in sample preparation will inspire the introduction of new advanced materials for the improvement of current analytical methods. Future work should be focused on the extension of this new synthetic strategy to the new MIPPy- $\mu$ SPE by imprinting different kinds of target molecules, for example biomolecules. It will be also interesting if more studies can be carried out to explore the possibility of applying this novel synthetic strategy in well-defined mono-disperse nanospheres in order to obtain a homogeneous population of binding sites. This synthetic strategy may be applied to make various biosensors. Our group is trying to employ this novel method to synthesize other functionalized nano-materials for biosensor research.

In this study, the modified syringe needle was able to be coupled to the commercially available HPLC autosampler. The integrated sample preparation/detection scheme allows the automation of selective extraction/cleanup of a red wine sample, followed by HPLC-FD analysis. It is noteworthy that the integration of sample preparation/detection with an HPLC autosampler did not need any additional accessories except a MISPE device. The automated set-up facilitated unattended operation and full instrument control via the autosampler's microprocessor. Once programmed, an operator can fill the autosampler tray with many/different samples and all following steps are controlled by the microprocessor. It requires no sample manipulation between the extraction and the HPLC analysis, therefore offering a high operating efficiency, improved precision, and good reproducibility. Furthermore, the affinity of MIPs toward specific target analytes makes this scheme more attractive in those areas where the selectivity or sensitivity is highly desired. Because the new preparation technique (developed in this thesis) can be 
applied to needles in various lengths and shapes, the resulting needles with different sizes can readily be used to handle small sample volumes (several $\mu \mathrm{L}$ ) as well as large volumes (several $\mathrm{mL}$ ). Thereafter, the modified needles can also be potentially coupled to a microfluidic system or liquid chromatography-mass spectrometry (LC-MS). In the present study, many parameters have been investigated due to the facility of the automated functions, such as the effect of template concentration, the choice of differential eluting solvents and the effect of analyte concentration. More work should be undertaken to further optimize the operating conditions and to better understand the binding mechanism of OTA toward MIPPy. For example, a study of binding/extraction kinetics could be achieved by changing the temperatures of the sample and eluting solution.

Additional experiments in our laboratory are underway to extend this new instrumental set-up for new applications in food, drug and environment analysis. 


\section{REFERENCES}


[1] O. BrÜggemann, K. Haupt, L. Ye, E. Yilmaz, K. Mosbach, J. Chromatogr. A 889 (2004): 15-24.

[2] C. Cacho, E. Turiel, A. Martin-Esteban, C. Pérez-Conde, C. Cámara, J. Chromatogr. B 802 (2004): 347-353.

[3] N. Masqueè, R.M. Marceè, F. Borrull, Trends Anal.Chem. 20 (2001): 477-486.

[4] O. Ramstrom, R. J. Ansel, Chirality 10 (1998): 195-209.

[5] F. Qiao, H. Sun, H. Yan, K. H. Row, Chromatographia 64 (2006): 625-634.

[6] M. Boopathi, M.V.S. Suryanarayana, A. K. Nigam, P. Pandey, K. Ganesan, B. Singh, K. Sekhar, Biosens. Bioelectron. 21 (2006) : 2339-2344.

[7] V. B. Kandimalla, H. Ju, Anal. Bioanal. Chem. 380 (2004): 587-605.

[8] M. Kawaguchi, Y. Hayatsu, H. Nakata, Y. Ishii, R. Ito, K. Saito, H. Nakazawa, Anal. Chim. Acta. 539 (2005): 83-89.

[9] G. Wulff, A. Sarhan, Angew. Chem., Intl. Ed. Engl. 11(1972): 341-345.

[10] T. Takagishi, IM. Klotz, Biopolymers 11 (1972): 483-491.

[11a] B. Sellergren, Molecularly Imprinted Polymers-Man-Made Mimics of Antibodies and Their Application in Analytical Chemistry, Elsevier, Amsterdam, 2001.

[11b] M.V. Polyakov, Zhur. Fiz. Khim. 2 (1931):799-807.

[12] A.G. Mayes, M. J. Whitcombe, (2005). Adv. Drug Delivery Rev. 57 (2005): 1742- 1778.

[13] C. Alexander, H. S. Andersson, L. I. Andersson, R. J. Ansell, N. Kirsch, I. A. Nicholls, J. ƠMahony, M. J. Whitcombe, J. Mol. Recognit. 19 (2006): 106-180.

[14] P. Spégel, L. Schweitz, S. Nilsson, Anal. Bioanal. Chem. 372 (2002): 37-38.

[15] J.O. Mahony, K. Nolan, M.R. Smyth, B. Mizaikoff, Anal. Chim. Acta. 534 (2002): 31-39.

[16] I. Yungerman, S. Srebnik, Chem. Mater. 18 (2006): 657-663. 
[17] K. Karim, F. Breton, R. Rouillon, E. V. Piletska, I. C. A. Guerreiro, S. A. Piletsky, Adv. Drug Delivery Rev. 57 (2005): 1795- 1808.

[18] L. Wu, K. Zhu., M. Zhao, Y. Li, Anal. Chim. Acta. 549 (2005): 39-44.

[19] T. Matsui, J Matsui, Acta Polymer. 47 (1996): 471-480.

[20] T. Takeuchi, T. Mukawa, H. Shinmori, The Chem. Record 5 (2005): 263-275.

[21] H. Zhang, L. Ye, K. Mosbach, J. Mol. Recognit. 19 (2006): 248-259.

[22] L. Ye, K. Mosbach, J. Inclusion Phenomena and Macrocyclic Chem.41(2001): $107-113$

[23] M. J. Whitcombe, E. N. Vulfson, Adv. Mater. 13 (2001): 467-478.

[24] B. Sellergren, Trends Anal.Chem. 18(1999): 164-174.

[25] I. Chianella, K. Karim, E. V. Piletska, C. Preston, S. A. Piletsky, Anal. Chim. Acta. 559 (2006): 73-78.

[26] Valérie Pichon, K. Haupt, Journal of Liquid Chromatography \& Related Technologies 29 (2006): 989-1023.

[27] L I. Andersson, Bioseparation 10 (2002): 353-364.

[28] D. Vaihinger, K. Landfester, I. Kra" uter, H. Brunner, G. E. M. Tovar, Macromol. Chem. Phys. 203(2002) 1965-1973.

[29] F. Schneider, S. Piletsky, E. Piletska, A. Guerreiro, M. Ulbricht, J. Appl. Polym. Sci. 98 (2005): 362-372.

[30] J. C.C. Yu, E. P. C. Lai, React. Funct. Polym. 66 (2006): 702-711.

[31] N. Perez-Moral, A. G. Mayes, Bioseparation 10 (2002): 287-299.

[32] I. S. Chronakis, A. Jakob, B. Hagstro, L. Ye, Langmuir 22 (2006): 8960-8965.

[33] R. M. Herna'ndez, L. Richter, S. Semancik, S. Stranick, T. E. Mallouk, Chem. 
Mater. 16 (2004): 3431-3438.

[34] Y. Tsai, S. Li, S. Liao, Biosens. Bioelectron. 22 (2006) : 495-500.

[35] M.A. Muñoz, M. Galán, L. Gómez, C. Carmona, P. Guardado, M. Balón, Chem. Phys. 290 (2003): 69-77.

[36] J.H. Chen, Z.P. Huang, D. Z. Wang, S. X. Yang, J. G. Wen, Z. F. Ren, Appl. Phys. A 73 (2001): 129-131.

[37] X. Lu , D. Chao, J. Chen , W. Zhang, Y. Wei, Mater. Lett. 60 (2006): 2851-2854.

[38] X. Cui, M. H. Engelhard, Y. Lin, J. Nanosci. Nanotechnol. 6 (2006): 547-553.

[39] M. Gao, L. Dai, G. G. Wallace, Synth. Met. 137 (2003): 1393-1394.

[40] J. C. C. Yu, E. P. C. Lai, Anal Bioanal Chem. 381 (2005): 948-952.

[41] S. Rimmer, Chromatographia 46 (1998): 470-474.

[42] A. Ramanavi cius, A. Ramanavi ${ }^{\smile ~ c i e n, ~ A . ~ M a l i n a u s k a s, ~ E l e c t r o c h i m i c a . ~ A c t a . ~}$ 51 (2006): 6025-6037.

[43] G. Z. Chen, M. S. P. Shaffer, D. Coleby, G. Dixon, W. Zhou, D. J. Fray, A. H. Windle, Adv. Mater 12 (2000): 522-526.

[44] C. Weidlich, K. M. Mangold, K. Ju“ ttner, Electrochimica. Acta. 47 (2001): $741-745$.

[45] G. Hana, J. Yuana, G. Shia, F. Wei, Thin Solid Films 474 (2005): 64-69.

[46] X. Zhang, J. Zhang, Z. Liu, Carbon.43 (2005): 2186-2191.

[47] W. M. Mullett, E. P. C. Lai, Anal. Chem. 70 (1998): 3636-3641.

[48] W. M. Mullett, E. P. C. Lai, J. Pharm. Biomed. Anal. 21(1999) : 835-843.

[49] S. G. Wu, E. P. C. Lai., P. M. Mayerb, J. Pharm. Biomed. Anal. 36 
(2004):483-490.

[50] C. Dall'Asta, G.. Galaverna, A. Dossena, R. Marchelli, J. Chromatogr. A 1024 (2004): 275-279.

[51] A. Bacaloni, C. Cavalier, A. Faberi, E. Pastorini, R. Samperi, A. Laganà, J. Agric. Food Chem. 53 (2005): 5518-5525.

[52] J. Blesa, J.M. Soriano, J.C. Moltó, J. Mañes, J. Chromatogr. A 1054 (2004): $397-401$.

[53] J. Varga, Z. Kozakiewicz, Trend. Food Sci. Tech. 17 (2006): 72-81.

[54] W. Ng, M. Mankotia, P. Pantazopoulos, Food Addit. Contam. 21 (2004): 971-981.

[55] A. Aresta, R. Vatinno, F. Palmisano, C. G. Zambonin, J. Chromatogr. A 1115 (2006): 196-201.

[56] D. Jornet, O. Busto, J. Guasch, J. Chromatogr. A 882 (2000): 29-35.

[57] J. Varga, Z. Kozakiewicz, Trend. Food Sci. Tech. 17(2006): 72-81.

[58] J. Jodlbauer, N. M. Maier, W. Lindner, J. Chromatogr. A 945 (2002): 45-63.

[59] N. M. Maier, G. Buttinger, S. Welhartizki, E. Gavioli, W. Lindner. J. Chromatogr. B 804 (2004): 103-111.

[60] E. González-Peñas, C. Leache, M. Viscarret, A. Pérez de Obanos, C. Araguás, A. López de Cerain, J. Chromatogr. A 1025 (2004): 163-168.

[61] J. C. C. Yu, S. Krushkova, E. C. Lai, Anal. Bioanal. Chem. 382 (2005): $1534-1540$.

[62] L. He, C. Toh, Anal. Chim. Acta. 556 (2006): 1-15.

[63] M. Trojanowicz, Trends Anal.Chem. 25 (2001): 480-489.

[64] Y. Cai, G. J., J. Liu, Q. Zhou, Anal. Chem. 75 (2003): 2517-2521. 
[65] G. Liu, J. Wang, Y. Zhu, X. Zhang, Anal. Letters. 37 (2004): 3085-3104.

[66] M. Valcárcel, B. M. Simonet, S. Ca' rdenas, B. Sua' rez, Anal. Bioanal. Chem. 382 (2005): 1783-1790.

[67] H. Okuno, T. Kitano, H. Yakabe, M. Kishimoto, B. A. Deore, H. Siigi, T. Nagaoka, Anal. Chem. 74 (2002): 4184-4190.

[68] Erik T. Thostensona, Z. Ren, T. Chou, Comp. Sci. Tech.61(2001): 1899-1912.

[69] Y. Xu, Y. Jiang, H. Cai, P.G. He, Y.Z. Fang, Anal. Chim. Acta 516 (2004):19-27.

[70] Y. Wei, L. Qiu, J. C.C. Yu, E. P.C. Lai, Food Sci. Tech. Int. (2007) article in press.

[71] X. Zhang, J.Zhang, R. Wang, T. Zhu, Z.and Liu, Chem. Phys. Chem. 5 (2004): $998-1002$.

[72] X. Liu, J. Ly, S. Han, D. Zhang, A. Requicha, M. E. Thompson, C. Zhou, Adv. Mater.17 (2005): 2727-2732.

[73] K. Teh, L. Lin, J. Micromech. Microeng. 15 (2005): 2019-2027.

[74] Y. Yu, C. Yang, Y. Gao, Z. Si, W. Chen, Z. Wang, G. Xue. J. Polym. Sci. A 43 (2005): 6105-6115.

[75] H. Valenta, J. Chromatogr. A 815 (1998): 75-92.

[76] J. C.C. Yu, E. P.C. Lai, Food Chem. (2006) article in press.

[77] Q. Xiao, X. Zhou, Electrochimica Acta 48(2003): 575-580.

[78] N. W. Turnera, E. V. Piletska, K. Karima, M. Whitcombe, M. Malecha, N. Magana, C. Baggianic, S. A. Piletsky, Biosens. Bioelectron. 20 (2004): 1060-1067.

[79] Y. V. Il'ichev, J. L. Perry, R. A. Manderville, C. F. Chignell, J. D. Simon, J. Phys. Chem. B 105 (2001) : 11369-11376.

[80] J.L. Sessler, G. Berthon-Gelloz, P.A. Gale, S. Camiolo, E.V. Anslyn, P. 
Anzenbacher, H. Furuta, G.J. Kirkovits, V.M. Lynch, Polyhedron 22 (2003) : 2963-2983.

[81] Y. Kanekiyo, R. Naganawa, H. Tao Angew. Chem. Int. Ed. 42 (2003): 3014-3016.

[82] N. Bell, S. Mar, V. Sanchis, A. J. Ramos, Food Sci. Tech.Int. 8 (2002): 325-335.

[83] C. J. Lin, T. Liedl, R. A. Sperling, M. T. Fernóndez-Argüelles, J. M. Costa-Fernaóndez, R. Pereiro, A. Sanz-Medel, W. H. Chang, W. J. Parak, J. Mater. Chem., 17 (2007): 1343-1346.

[84] Y. J. Gong, Progress in Chem. 19 (2007): 502-509.

[85] R.C. Somers, M.G. Bawendi, D.G. Nocera, Chem. Soc. Revi. 36 (2007): 579-591.

[86] www.nature.com/nprot/journal/v1/n3/full/nprot.2006.184.html

[87] www.physorg.com/news536.html

[88] www.sciencemag.org/cgi/content/full/300/5616/80

[89] www.sciencemag.org/cgi/content/full/307/5709/538

[90] W. Itamar, B. Ronan, W. Bilha, Biosens. Bioelectron. 22 (2007): 1841-1852.

[91] W. Itamar, B. Ronan, W. Bilha, Febs J. 274 (2007): 302-309.

[92] C. Huang, Y. Li, T. Chen, Biosens. Bioelectron. 22 (2007): 1835-1838.

[93] L. Niu, W. Knoll, Anal. Chem., 79 (2007): 2695-2702.

[94] J.M. Perkel, Scientist 16 (2002): 32-34.

[95] scienceweek.com/2003/sw030321.htm

[96] materials.globalspec.com/.../Electrical_Nanomaterials_Nanotechnology_Products - 60k - .

[97] www.nature.com/nmeth/journal/v1/n1/full/nmeth032.html.

[98] www.cdc.gov/niosh/topics/nanotech/safenano/healthconcerns.

[99] E.H. Sargent, Adv. Mater. 17 (2005): 515-522. 
[100] www.mrs.org/s_mrs/bin.asp?CID=2629\&DID=61013\&DOC=FILE.PDF

[101] X. Gao, W.C.W. Chan, S. Nie, J. Biomed. Opt., 7 (2002): 532-537.

[102] http://www.esm.psu.edu/news/item/274

[103] P.G. Su, Y.L. Sun, C.C. Lin, Sen. Actuators: B 115 (2006): 338-343.

[104] M. Olek, M. Hilgendorff, M. Giersig, Colloid. Surf. 292 (2007): 83-85.

[105] S. Ravindran, K.N. Bozhilov, C.S. Ozkan, Carbon 42 (2004) 1537-1542.

[106] P. Li, X. Lim, Y. Zhu, T. Yu, C. Ong, Z. Shen, A. Wee, C. Sow, J. Phy. Chem. B 111 (2007): 1672-1678.

[107] Y. Xiao, J. Che, C. Li, C.Q. Sun, Y. T. Chua, V. S. Lee, J. H. T. Luong, J. Biomedic. Mater. Research A 80A (2007): 925-931.

[108] H. Peng, C. Soeller, J. H. Travas-Sejdic, Macromolecules 40 (2007): 909-914.

[109] G. Theodoridis, I. N. Papadoyannis, Mikrochim. Acta 136 (2001): 199-204.

[110] G. A. Theodoridis, C. K. Zacharis, A. N. Voulgaropoulos, J. Biochem. Biophy. Meth. 70 (2007): 243-252.

[111] G. Theodoridis, C. K. Zacharis, P. D. Tzanavaras, D. G. Themelis, A. Economou, J. Chromatogr. A 1030 (2004): 69-76.

[112] A. Wang, F. Fang and J. Pawliszyn, J. Chromatogr. A 1072 (2005): 127-135.

[113] C. Brera, J.M. Soriano, F. Debegnach, M. Miraglia, Microchem. J. 79 (2005): $109-113$.

[114] J.J. Gooding, C. Wasiowycha, D. Barnett, D.B. Hibbert, J.N. Barisci, G.G. Wallace, Biosens. Bioelectron. 20 (2004): 260-268.

[115] S. Sadki, et al, Chem. Soc. Rev. 29 (2000): 283-293.

[116] X. Zhang, R. Bai, Langmuir 19 (2003): 1073-1079. 
[117] H. S. Andersson, J. G. Karlsson., S. A. Piletsky, A. Koch-Schmidt , K. Mosbach, I. A. Nicholls, J. Chromatogr. A 848 (1999): 39-49.

[118] icon.rice.edu/resources.cfm?doc_id $=4394$

[118] Teresa Walker, J. Morris, R. Threlfall, G. Main, J. Agric. Food Chem 51 (2003): 1543-1547.

[119] R.C. Rodriguez-Diaz, M. P. Aguilar-Caballos, A. Gomez-Hens, J. Sep. Sci. 29 (2006): 2772-2779.

[120] P. Varelis, S.L. Leong, A. Hocking, G. Giannikopoulos, Food Addit. Contam. 23 (2006): 1308-1315.

[121] S.Y. Feng, E. P. C. Lai., E. Dabek-Zlotorzynska, S. Sadeghi, J. Chromatogr. A 1027 (2004): 155-160.

[122] M.A. Rodriguez-Delgado, S. Malovana, J.P. Perez, T. Borges, F.J. Garcıa Montelongo, J. Chromatogr. A 912 (2001): 249-257.

[123] S.A.S.S. Gomes, J. M. F. Nogueira, M.J.F. Rebelo, Biosens. Bioelectron 20 (2004): 1211-1216.

[124] M. Rodríguez-Delgado, G. González-Hernández, J. Conde-González, J. Pérez-Trujillo, Food Chem. 78 (2002): 523-532. 


\section{Appendix 1}

Bio-Rad Model AS-100 HRLC Automatic Sampling System

Operation Manual 


\section{Overview}

The Model AS-100 HRLC automatic sampling system is a flexible processor.

The main components of the autosampler are (illustrated in Fig. A1):

1) six-port injection valve with sample loop (the current sample loop is $20 \mu \mathrm{L}$, it is changeable);

2) Sample needle assembly including injection needle (one original, or any $22 \mathrm{G} 1.5$ BD stainless steel syringe needle), connection tubing (extended to the injection valve); To connect the MISPE sorbent modified needle, a short length of Nalgene connection tubing was used to mount up the modified needle. It was necessary to equip the connection tubing with a septum that helped avoid sample leaking as well as minimize band broadening during the transfer of sample solution from the vial to the injection loop.

3) Injection needle mechanical arm;

4) Sample table including sample tray (10X10, only 10X5 vials shown in Fig. A1 ), wash well, and tray well;

5) Control (front) panel;

6) Power switch (on the back, not shown)

In this manual, two operation models (direct HPLC analysis and automated $\mu$ MISPE-HPLC analysis) as well as several functions provided by the autosampler are introduced. In order to better understand the autosampler, these functions are explained before the introduction of the two operation models.

In section A-2, two different loop filling methods are introduced, they are complete filling method and partial filling method. In section A-3 and section A-4, two independent functions are explained respectively, they are manual injection function and flush function. In section A-5 and section A-6, two operation models are introduced AND they 
are direct HPLC analysis (section A-5) and automated $\mu$ MISPE -HPLC analysis (section A-6). In the introduction of direct HPLC analysis, all the tables appeared on the control panel are shown and the parameters are explained in the sequence of operation. In the introduction of $\mu \mathrm{MISPE}-\mathrm{HPLC}$ analysis, the operation steps are listed with some explanation.

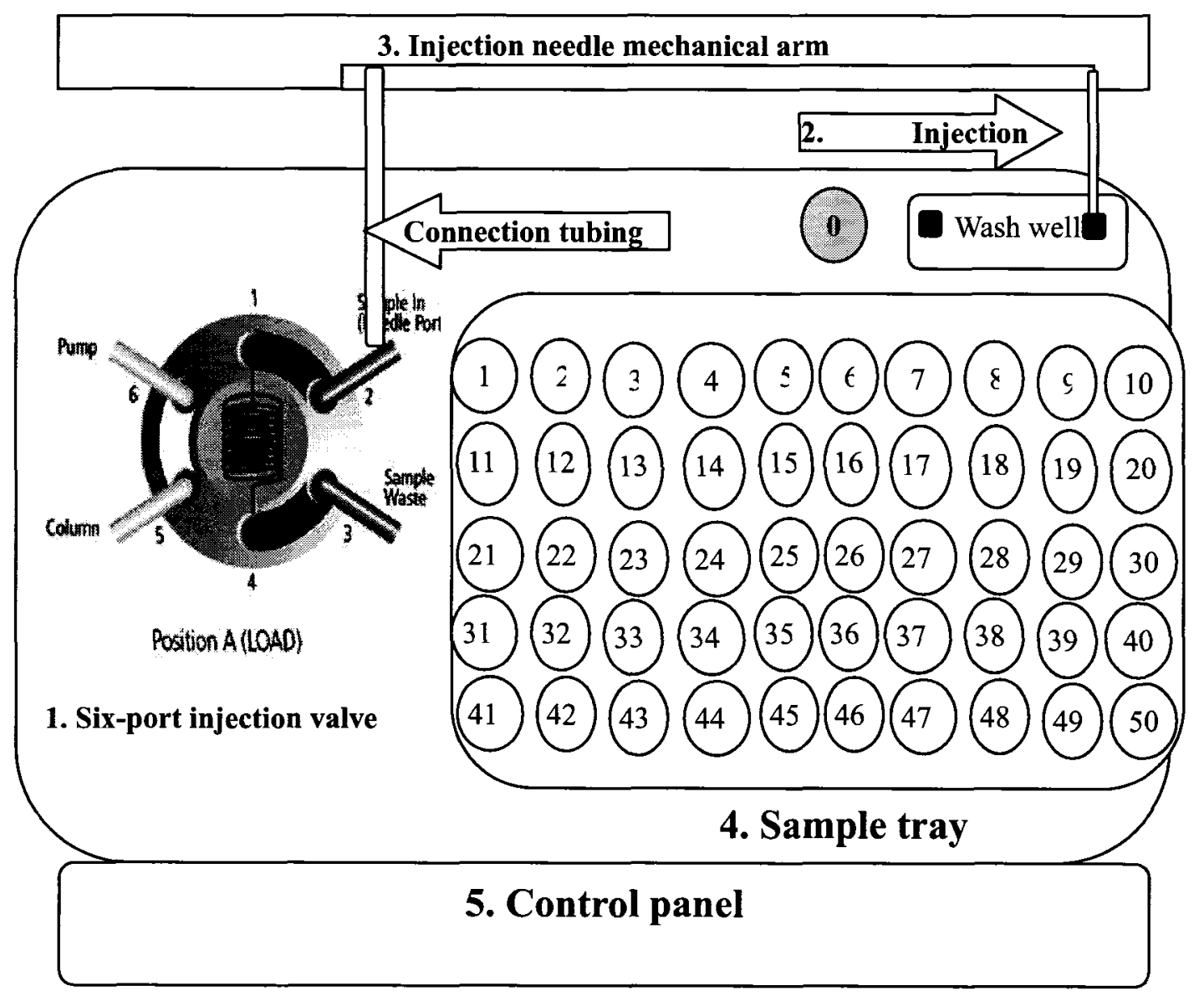

Fig. A-1 Diagram of AS-100 HRLC automatic sampling system

\section{Sample loop filling method}

The autosampler provides two loop filling method and variable injection volume (it allows the loading of different sample volumes). In this thesis research, the complete filling method was used for direct HPLC analysis and sample uptake while partial filling 
method was used for eluting extracted OTA from the needle.

\subsection{Complete filling method}

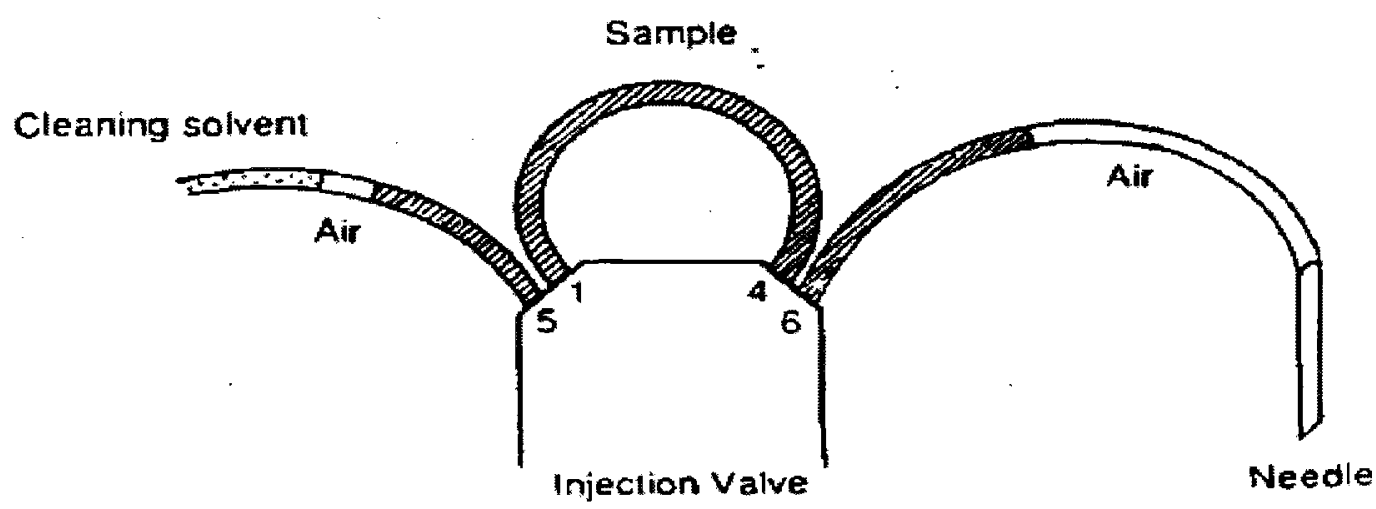

Fig. A-2 Fluid path in complete loop filling operation

In the complete loop filling method (illustrated in Fig. A-2), wash solvent (DDW) is first flushed through the sample loop and injection needle, then a fixed volume of sample (injection volume + overfill volume) is drawn through the injection needle and loaded onto the sample loop. After the sample injected onto the HPLC column, wash solvent (DDW) will flush the sample loop and injection needle again in order to avoid any carryover from the previous injection. The autosampler will determine whether complete filling operation or partial filling method performs (see section A-5.2, sample loop size). If complete filling operation is chosen, the system injects the sample according the sequence shown in Fig. A-3.

In this filling method, a pocket of air is drawn while the sampling needle is held above the sample well (as shown in Fig. A-3). The pocket of air is introduced into the sample tube to provide an interface through which mixing of liquids cannot occur. Thus, the concentration of the sample is not altered by wash solvent dilution, and accuracy is maintained. 


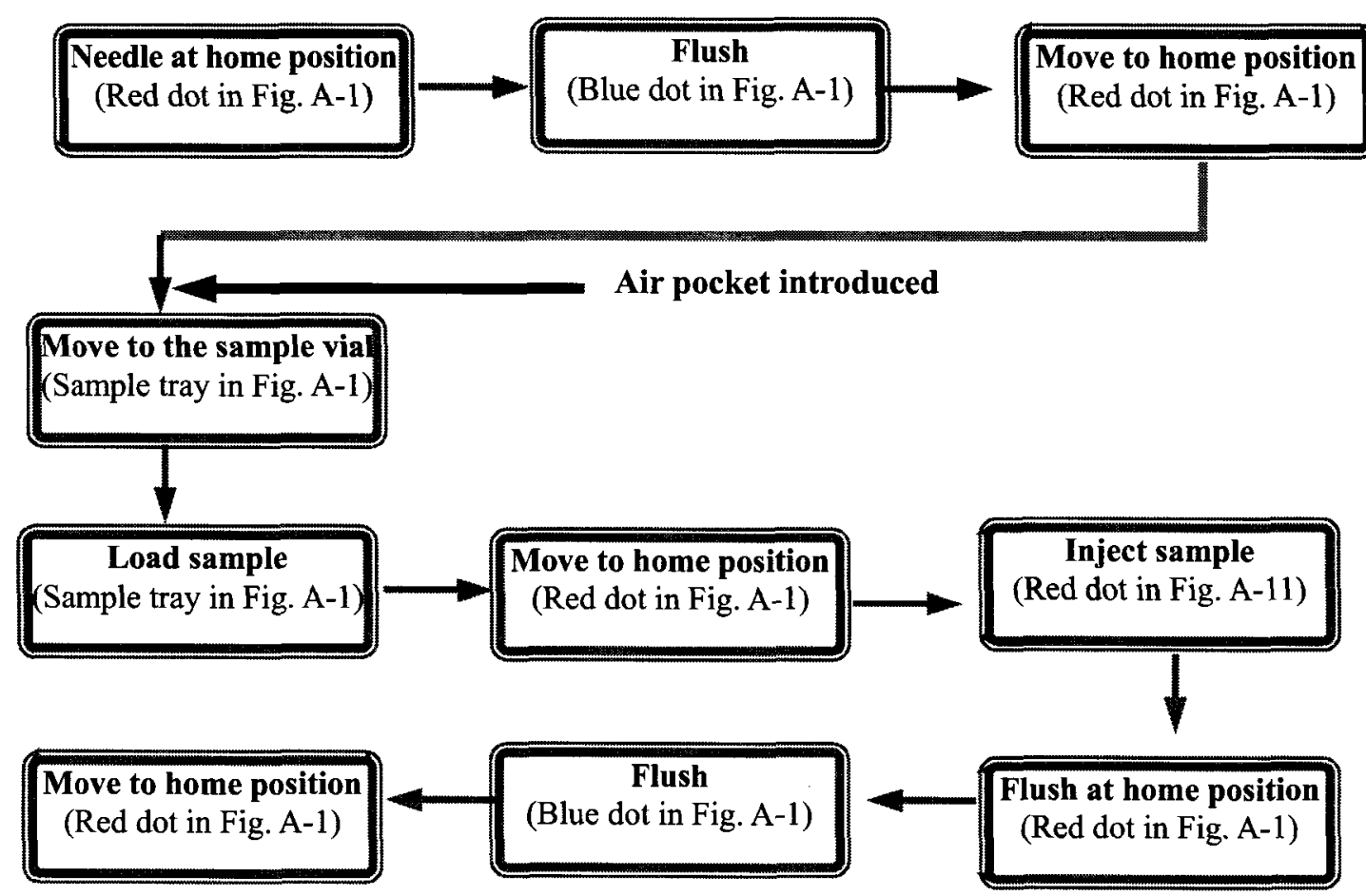

Fig. A-3 Flow chart of the complete filling method

In this filling method, a pocket of air is drawn while the sampling needle is held above the sample well (as shown in Fig. A-3). The pocket of air is introduced into the sample tube to provide an interface through which mixing of liquids cannot occur. Thus, the concentration of the sample is not altered by wash solvent dilution, and accuracy is maintained.

\subsection{Partial loop filling method}

In the partial loop filling method, a fixed volume of (injection volume + overfill volume) sample is drawn (as illustrated in Fig. A-4), first by bypassing the sample loop. Then a sample volume less than the total volume of the sample loop is introduced to the loop for injection. The flush follows the injection. The sequence is shown in Fig. A-5. 


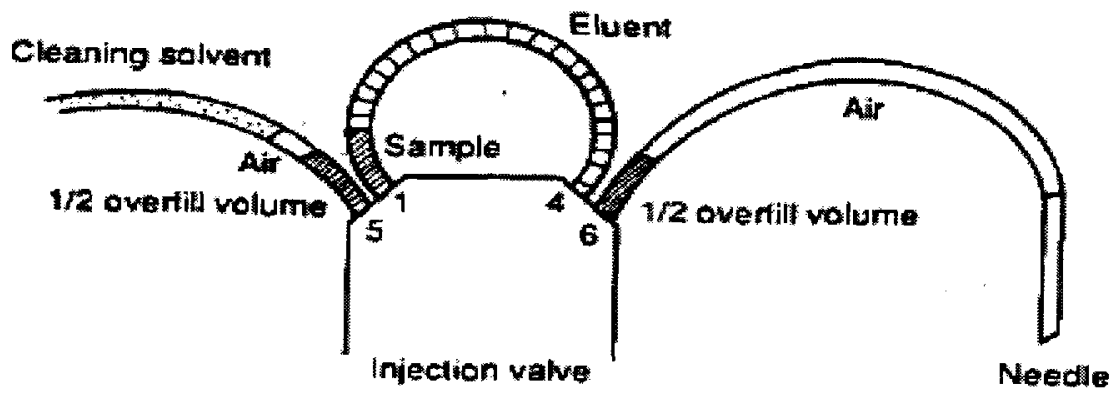

Fig. A-4 Fluid path in partial loop filling method

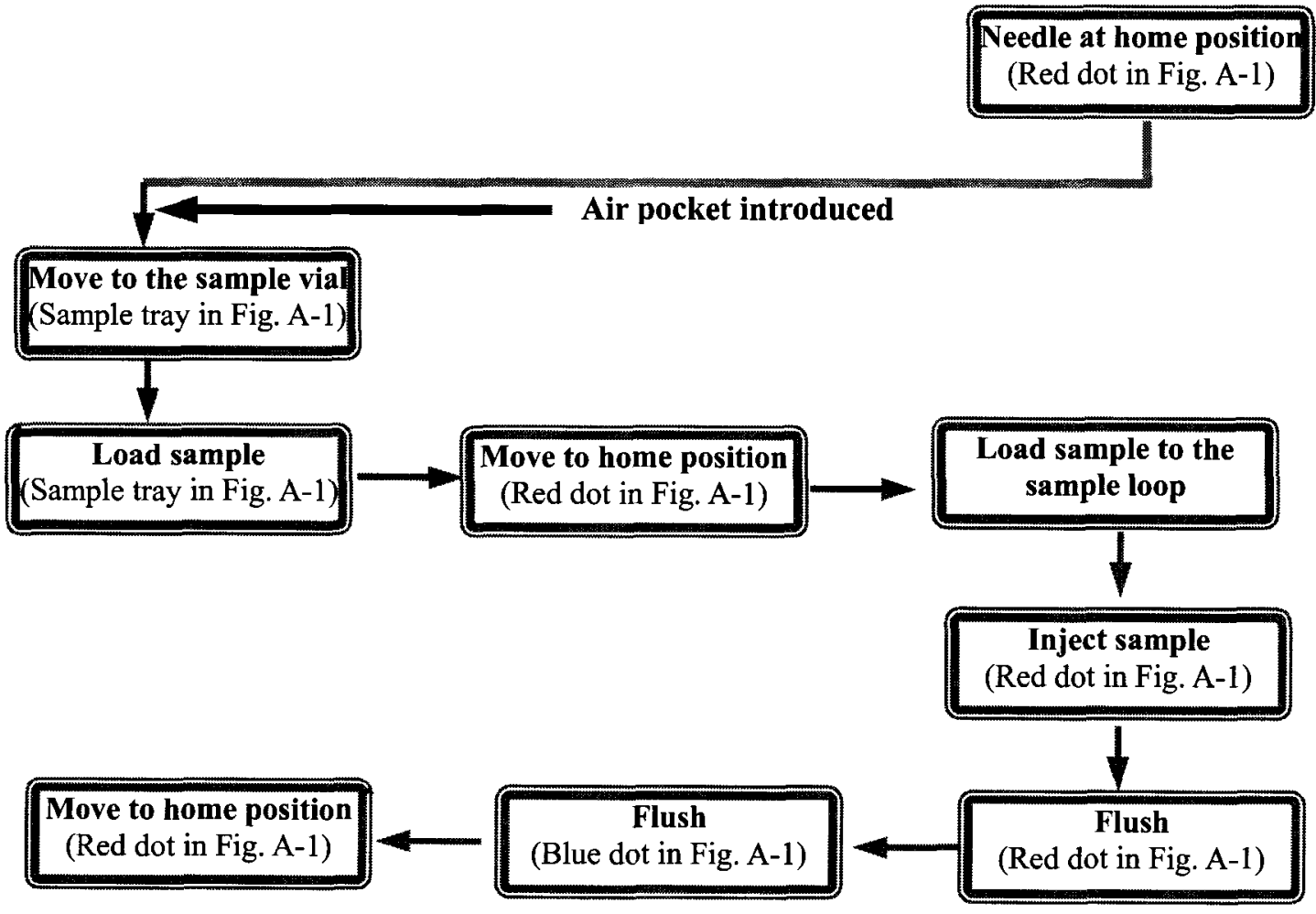

Fig. A-5 Flow chart of the partial filling method 


\section{Manual injection function}

Status: STOP

Manual Injection

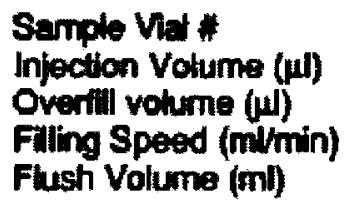

Fig. A-6 Manual injection table

The manual injection function is available to inject a single sample, or a priority sample while a sample table run is in operation. The manual injection table is shown in Fig. A-6 and the parameters are explained as following:

1) Sample vial number: the position from which one sample will be drawn for one injection. Any vial position (1-100) of the sample tray could be chosen in addition to the "0" position of the sample tray (shown in Fig. A-1).

2) Injection volume: explained in A-5.3 3)

3) Overfill volume: explained in A-5.2 5)

4) Filling speed: explained in A-5.2 4)

5) Flush volume: see explained in A-5.2 6)

\section{Flush functions}

Flush function is used to wash the sample needle assembly (shown in Fig. A-1). In the automated $\mu$ MISPE-HPLC analysis, the flush should be conducted after each uptaking of $1.0 \mathrm{~mL}$ sample to eliminate any carry over. Three times flush is more than enough to avoid any carryover. 
Flush function is used to wash the sample needle assembly (shown in Fig. A-1). In the automated $\mu$ MISPE-HPLC analysis, the flush must be conducted after each uptaking 1.0 $\mathrm{mL}$ sample to eliminate any carry over. Three times flush is more than enough to avoid any carry over.

To use the flush function, press the "Function" key and then the "Flush" key. The flush screen will appear (see Fig. A-7). It could be programmed from $0.1 \mathrm{~mL}$ to $1.0 \mathrm{~mL}$ in $0.1 \mathrm{~mL}$ increments. Accept the default flush volume of $1.0 \mathrm{~mL}$. Press the "Start" key. The injection needle will move down into the wash well (blue dot position in Fig. A-1) and draw wash solvents (DDW) from the wash solvent reservoir. During this step, the sample needle assembly (including connection tubing and injection needle) is washed with the DDW.

\section{Operation steps of direct HPLC analysis}

Status: STOP

System Flush

Flush Volume (m)

Fig. A-7 Flush table

The concept of direct HPLC analysis is very similar to manual HPLC analysis, except the analysis is done automatically.

\subsection{Switch on the power and self-check}

When the power is switched on, the system is first self checked, which includes: 1) back-up battery check; 2) injection valve check; 3) x-y axes moving check; 4) z-axis moving check; 5) syringe pump moving check. 
In the step of 3), the injection needle mechanical arm will move along $\mathrm{x}$ and $\mathrm{y}$ axes and then the arm will bring the injection needle back to the position of the wash well (the red dot position in Fig. A-1, which is the home position of the needle). In the step of 4), the injection needle first moves to the blue dot position in Fig. A-1 (the washing position) of the wash well. Then the injection needle mechanical arm will move the needle up and down which is called "the z-axis moving check". (The self-check sometimes fails at this step if the injection needle is not installed properly).

The whole process will take about 5 minute. On the control panel the time remaining will be shown.

After self-checking, the system is ready for use.

\subsection{Set up the system configurations}

The system configuration screen establishes the operating conditions of the autosampler by setting the default values of the other operation screens. The screen comes up automatically after the self-check sequence has been completed at power-up. The screen can also be manually assessed by first pressing the "Function" key (shown in Fig. A-8), then press the "Set Sys. 8" key (shown in Fig. A-8). When you change the setting of the system, first enter the corresponding digit by pressing the digital keys, and then press the "enter" key. 
In the system configuration screen, some parameters of the system can be changed, such as the size of the sample loop, temperature, filling speed, overfill volume and flush volume. All these parameters are shown in Fig. A-9 and explained as following:

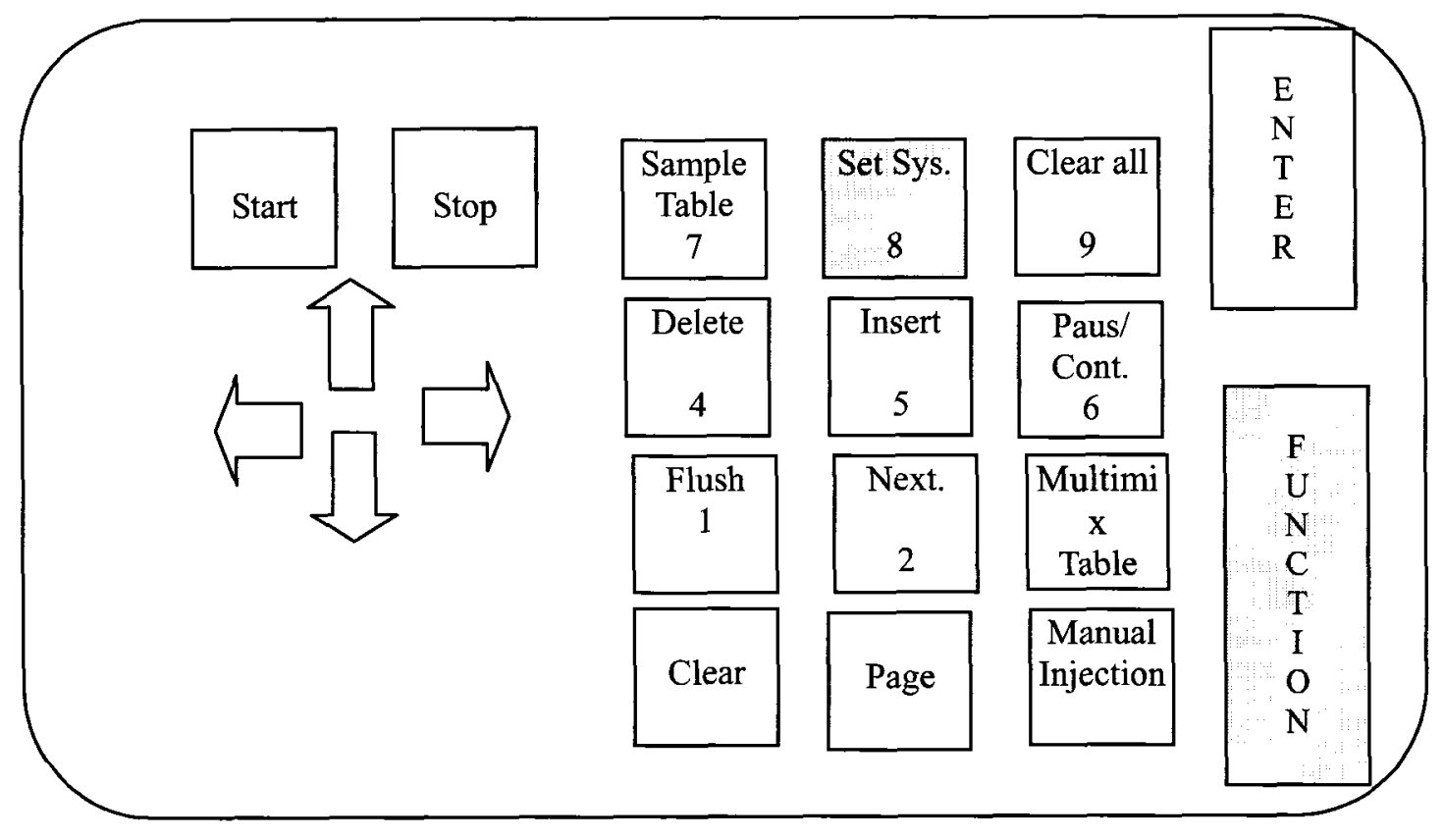

Fig. A-8 Diagram of the control panel key board

Status: STOP

System Configuration Parameters

Semple Table Directory

Sample Loop Size

Thermal Module

Temperature

Ftling Speed

Overtill Volume

Flush Volume

Remote Mode

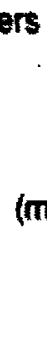

(1)):

$\left({ }^{\circ} \mathrm{C}\right)$

(milmin):

(ul):

(mi):

:

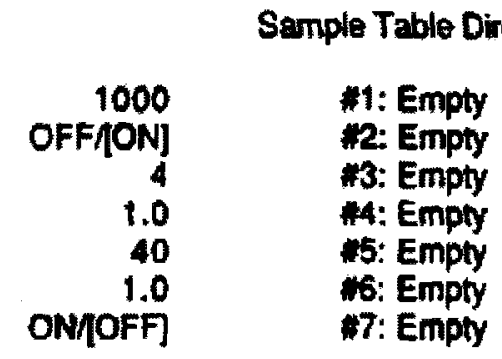

Fig. A-9 System configuration screen 
1) Sample loop size: the permissible sample loop sizes are $5,10,20,50,100,200,500$, and $1000 \mu \mathrm{L}$.

The sample loop size entered on the system configuration screen is compared with the injection volume to determine whether the complete loop filling or partial loop filling method will be executed. For manual injection, injection volume can be entered in the "manual injection screen". (for manual injection screen see section A-3) ; for unattended injection, injection volume is entered in the sample sequence screen table, see section A-4.3.

2) Thermal module: when the thermal module is "ON", the sample tray well will be cooled to the temperature entered in the temperature field. Cooling is programmable from $2^{\circ} \mathrm{C}$ to $35^{\circ} \mathrm{C}$ in $1{ }^{\circ} \mathrm{C}$ increments. The left and right arrow keys are used to switch between the two settings.

3) Temperature: enter the desired temperature for you purpose (from $2^{\circ} \mathrm{C}$ to $35^{\circ} \mathrm{C}$ in $1^{\circ} \mathrm{C}$ increments).

4) Filling speed: the draw rate of sample into sample loop. The user may enter a filling speed in the range of 0.1 to $2.0 \mathrm{~mL} / \mathrm{min}$. Filling speed is programmable in increments of 0.1 . The default value is $1.0 \mathrm{~mL} / \mathrm{min}$.

5) Overfill volume: the injection volume plus the overfill volume is drawn from the sample vial and delivered to the sample loop. Very similar to the manual HPLC injection, the sample loop is $20 \mu \mathrm{L}$ but $50 \mu \mathrm{L}$ sample is loaded into the sample loop, the overfill volume goes to the waste. The minimum overfill volume accepted by the autosampler is $10 \mu \mathrm{L}$ while the maximum is $200 \mu \mathrm{L}$. The default value is $40 \mu \mathrm{L}$. Overfill is programmable only in $1 \mu \mathrm{L}$ increments. 
6) Flush volume: controls the amount of wash solvent (DDW) that flushed the sample loop and sample needle assembly (including connection tubing and injection needle). A flush volume in the range of 0.0 to $4.0 \mathrm{~mL}$ could be selected and entered. Flush volume is programmable in $0.1 \mathrm{~mL}$ increments. The default value of $1.0 \mathrm{~mL}$ is believed to be more than adequate to prevent carry over of sample between injections.

\subsection{Set up the sample sequence table}

Setting up the sample sequence table provides unattended, automatic sequencing and injection of sample. To reach the sample table screen, press the "Function "key, and then the "Sample Table 7" key. Select the sample table number, and then press the "Enter" key to reach the appropriate sample sequence table. A sample sequence table is shown in Fig. A-10 and explained as following:

Status: STOP

\begin{tabular}{|c|c|c|c|c|c|c|c|c|}
\hline \multirow{2}{*}{$\begin{array}{l}\text { Sample Sequence Table *2 } \\
\text { Sequence * }\end{array}$} & \multicolumn{3}{|c|}{ Tray: $100 \times 1.5 \mathrm{~m}$} & \multicolumn{2}{|c|}{$100 \times 0.5 \mathrm{~m}$} & \multicolumn{2}{|c|}{ Microptate } & \multirow[b]{2}{*}{8} \\
\hline & 1 & 2 & 3 & 4 & 5 & 6 & 7 & \\
\hline First Vial Postion & $=$ & - & - & - & - & - & $=$ & - \\
\hline Last Vial Position & - & - & - & - & - & - & • & - \\
\hline Injection Volume (ul) & - & - & $=$ & - & - & - & - & - \\
\hline - Injections per vial & - & - & - & - & - & - & - & - \\
\hline Overtil Volume (ul) & - & - & - & $=$ & $=$ & - & - & - \\
\hline Filing Speed (mi/min) & - & - & = & - & * & - & = & * \\
\hline Flush volumo (m) & - & - & - & - & - & - & - & - \\
\hline Cycle Time & - & - & - & - & - & - & - & $=$ \\
\hline
\end{tabular}

Fig. A-10 The sample sequence table

1) First vial position: the first vial to be run in a sequence.

2) Last vial position: the last vial to be run in a sequence. If only one vial is to be sampled, the last vial number is the same as the first vial number. 
3) Injection volume: determines whether complete or partial loop filling operation will be performed. If the injection volume matches the sample loop volume on the system configuration screen, then complete loop filling operation will take place. If the injection volume is less than the sample loop volume entered in the system configuration screen, then partial loop filling operation will take place.

4) \# injections per vial: the number of injections or repetitions for a given sequence.

5) Overfill volume: same in A-5.2 5)

6) Filling speed: same in A-5.2 4)

7) Flush volume: same in A-5.2 6)

8) Cycle time: the cycle time holds the time in minutes between sample injections. The time entered usually represents the run time of the chromatographic run. However, sufficient time must be allowed for the automatic sampler to perform its sampling, injection, and flush routines. The user should enter a time greater than or equal to, the sum of the chromatographic run time plus any required column equilibration or data station report printing period.

\subsection{Start}

Once the above parameters are set, you can press the "Start" key to get started. Before start, make sure that there is enough sample solution in the sample vial.

When the program is finished, it stops automatically. By pressing the "Stop" key, the program can be stopped manually during the operation and the needle will be cleaned by flushing with DDW.

\section{Automated $\mu$ MISPE -PE/DPE-HPLC-FD analysis}

Automated $\mu \mathrm{MISPE}-\mathrm{PE} / \mathrm{DPE}-\mathrm{HPLC}-\mathrm{FD}$ analysis was used to sampling 
(extraction)/pulsed elution/injection (detection) of OTA in a sample using MIPPy-nanocomposite-modified needle for sample injection.

\subsection{Sampling (extraction)}

\subsubsection{Set the system configurations}

For automated $\mu$ MISPE-HPLC analysis, the first step is sample uptake. At this step the complete filling operation is performed.

Use the system configurations (see section A-4.2) to set up all the following parameters as shown in Table 1 for sample uptake.

Table A-1 System configuration at the sample uptake step (1.0 mL sample)

\begin{tabular}{|l|l|}
\hline Parameters & Settings \\
\hline Sample loop size & $1000 \mu \mathrm{L}$ \\
\hline Thermal module & ON \\
\hline Temperature & $21^{\circ} \mathrm{C}$ \\
\hline Filling speed & $0.1 \mathrm{~mL} / \mathrm{min}$ \\
\hline Overfill volume & $10 \mu \mathrm{L}$ \\
\hline The flush volume & $0.1 \mathrm{~mL}$ \\
\hline
\end{tabular}

In the automated $\mu \mathrm{MISPE}$, a sample loop size of $1000 \mu \mathrm{L}$ in the sample uptake step was set for the purpose of drawing $1000 \mu \mathrm{L}$ sample ( OTA standard solution or red wine ) through the $\mu$ MISPE sorbent modified needle in spite of the fact that a $20 \mu \mathrm{L}$ sample loop was installed in the system. Once the sample uptake step is done, the sample loop size is 
set back to $100 \mu \mathrm{L}$ designed for pulsed elution.

In the $\mu$ MISPE, setting different filling speeds could change the sample uptake flow rate. For OTA analysis, it is optimized to be $0.1 \mathrm{~mL} / \mathrm{min}$.

\subsubsection{Uptake the sample through the $\mu$ MISPE sorbent modified needle}

Put the sample vial with the sample solution in the right position of the sample try.

After all the parameters are set up, you can start by just press "Start" key (shown in Fig. A-8. The injection needle in complete filling method will move according to the sequence shown in Fig. A-3.

After the sample is loaded into the sample loop, watch the control panel. Once "injection" shows on the screen, the user can start the integrator or any other data acquisition system. Some "click" sound will be heard when the six-port sample loop (shown on Fig. A-1) rotates from the "load position" to the "inject position". The user should start the integrator or any other data acquisition system at the same time you hear the "click" sound when the six-port sample loop rotates to the "inject position".

The sample can be loaded onto the HPLC column or it can bypass the HPLC column. If the $1.0-\mathrm{mL}$ red wine sample is used as the working solution, bypassing the HPLC column is recommended. If you choose to bypass the HPLC column, the user must manually/physically disconnect the autosampler from the HPLC column before uptaking

the sample. After the sample uptake, wash the sample loop with HPLC mobile phase for about 3 minutes, and then connect the autosampler with HPLC column again. Let the mobile phase flush the sample loop, HPLC column and detector tubing for about 7 minutes. During this period of time, run the integrator to monitor if there are any residual peaks from previous red wine sample. 
After sample uptake, run "Flush" function (see section A-4) for 3 times in order to eliminate any carry over in the injection needle tubing.

\subsection{Pulsed elution/differential pulsed elution}

Put the vial with eluting solvent in the " 1 " position of the sample tray.

For pulsed elution or differential pulsed elution, the manual function (see section A-3) is utilized and the partial loop filling method is performed. The partial loop filling method is used for eluting extracted OTA from the MIP modified needle since the needle will be washed with DDW first in the complete filling method. DDW was found to be able to elute the extracted OTA from the $\mu$ MISPE sorbents

Use the system configurations screen (see in section A-4.2) and manual injection table (shown in Fig. A-6) to set up all the following parameters (as shown in Table 2 and Table 3) for pulsed elution.

When all parameters are set, the user can start by pressing the "Start" key (shown on Fig. A-6). For differential pulsed elution, you can press the "Start" key after the previous pulsed elution is done.

\subsection{Injection (detection)}

After you start the program and the sample is loaded onto the sample loop, keep watching the control panel. Once "injection" shows on the screen, the user should start the integrator or any other data acquisition system at the same time. Some "click" sound will be heard when the six-port sample loop (shown in Fig. A-1) rotates from the "load position" to the "inject position". The user should start the integrator or any other data acquisition system at the same time as you hear the "click" sound. 
Table A-2 System configuration for pulsed elution

(0.2 mL eluting solvent each time)

\begin{tabular}{|l|l|}
\hline Parameters & Settings \\
\hline Sample loop size $(\mu \mathrm{L})$ & 100 \\
\hline Thermal module & ON \\
\hline Temperature $\left({ }^{\circ} \mathrm{C}\right)$ & 21 \\
\hline Filling speed $(\mathrm{mL} / \mathrm{min})$ & 1.0 \\
\hline Overfill volume $(\mu \mathrm{L})$ & 150 \\
\hline Flush volume $(\mathrm{mL})$ & 0.2 \\
\hline
\end{tabular}

Table A-3 Manual injection table for pulsed elution

(0.2 mL eluting solvent each time)

\begin{tabular}{|l|l|}
\hline Parameters & Settings \\
\hline Sample vial \# & 1 \\
\hline Injection volume $(\mu \mathrm{L})$ & 50 \\
\hline Overfill volume $(\mu \mathrm{L})$ & 150 \\
\hline Filling speed $(\mathrm{mL} / \mathrm{min})$ & $1.0 \mathrm{~mL} / \mathrm{min}$ \\
\hline Flush volume $(\mathrm{mL})$ & 0.1 \\
\hline
\end{tabular}

For pulsed elution, an overfill volume of $150 \mu \mathrm{L}$ is set (the volume of the pulsed eluting solvent=injection volume $50 \mu \mathrm{L}+$ overfill volume $150 \mu \mathrm{L}=200 \mu \mathrm{L}$ ) 


\section{Appendix 2}

\section{Publications and Presentations}

Y. Wei, L. Qiu, C. Owen; E. P. C. Lai, "Encapsulation of Quantum Dots and Carbon Nanotubes with Polypyrrole in a Syringe Needle for Automated Molecularly Imprinted Solid Phase Pre-concentration of Ochratoxin A in Red Wine Analysis". Accepted by Sensing and Instrumentation for Food Quality and Safety.

Y. Wei, L. Qiu, J. C.C. Yu and E. P. C. Lai, "Molecularly Imprinted Solid Phase Extraction in a Syringe Needle packed with Polypyrrole-encapsulated Carbon Nanotubes for Determination of Ochratoxin A in Red Wine". Accepted by Food Science and Technology International.

Y. Wei, E. P. C. Lai, “ Molecularly imprinted polypyrrole-carbon nanotubes modified stainless steel needles for selective solid phase extraction in trace analysis of ochratoxin A", poster \#39, Ottawa-Carleton Chemistry Institute Day, Carleton University, May, 2006.

Y. Wei, L. Qiu, E. P. C. Lai, “Automated Molecularly Imprinted Solid Phase Extraction in a Syringe Needle packed with Polypyrrole encapsulated Carbon Nanotubes for Determination of Ochratoxin A in Red Wine" , poster \# 303, 90th Canadian Chemistry Conference, Winnipeg, Manitoba, Canada, May 26-30, 2007.

E. P. C. Lai, Y. Wei, M. Pereira, Z. de Maleki, "Molecularly imprinted polymer-modified nanomaterials for chemical sensing of trace mycotoxins and estrogens", oral presentation, 90th Canadian Chemistry Conference, Winnipeg, Manitoba, Canada, May 26-30, 2007. 